\title{
Precision strength training: Data-driven artificial intelligence approach to strength and conditioning
}

\author{
Petteri Teikari* \\ High-Dimensional Neurology Group, \\ University College London Queen Square Institute of Neurology, London, UK \\ Aleksandra Pietrusz \\ MRC Centre for Neuromuscular Diseases, \\ University College London Queen Square Institute of Neurology, London, UK
}

(Dated: May 20, 2021)

\begin{abstract}
In strength training, personalised strength training (autoregulation) approaches have been used to individualise exercise programs with monitoring for dynamic adjustment based on the individual response to training. While this transition from tradition-based training to evidence-based training framework has been an improvement in training practices, we argue that the future of strength training will also incorporate deep learning models powered by data. We refer to this data-driven framework as precision strength training inspired by the similar modeling frameworks used in precision medicine. In contrast to current personalised training in which the acquired athlete data is often subject to human expert decision-making, we are anticipating the rise of human-in-the-loop systems with an augmented coach who will be doing decisions collaboratively with the machine. Similar to other precision frameworks, such as precision health, we envision such a future to take decades to be realised and we focus here on practical short-term targets on a way to long-term realisation. In this chapter, we will review the measurement technology needed for continuous data acquisition from an individual during training/physical activity, how to acquire these datasets for the development of such systems and, how a proof-of-concept system could be developed for powerlifting training with applicability to general strength and conditioning $(\mathrm{S} \& \mathrm{C})$ and physical rehabilitation purposes. Additionally, we will evaluate how the user experience (UX) of the system feedback and visualisation could be designed.
\end{abstract}

Keywords: artificial intelligence, machine learning, deep learning, precision medicine, resistance training, strength training, precision strength training, strength and conditioning, powerlifting, physical rehabilitation

Authors agreement statement: All authors have approved the final version of this manuscript and have agreed to its submission to a pre-print server. Petteri Teikari (@petteriTei) can be reached on Twitter.

This document is a pre-print submitted to SportRxiv

Preferred citation: Teikari, Petteri, and Aleksandra Pietrusz. 2021. "Precision Strength Training: Data-driven Artificial Intelligence Approach to Strength and Conditioning." SportR $\chi i v$. May 20. doi: 10.31236/osf.io/w734a

\section{INTRODUCTION AND BACKGROUND}

The massive amount of sports data generated has enabled the use of more and more powerful artificial intelligence (AI) models for the analysis of individual athlete performance, team play strategies and fans engagement [28, 126, 242, 713, 715]. For example, Christina Chase from MIT Sports Lab argues that: "data is the currency by which competitive advantage is won and lost.

\footnotetext{
* petteri.teikari@gmail.com
}

Those who find creative ways to unlock and harness it - will be the champions of tomorrow' [126]. Despite big data having been extensively used for in-game decision making in professional sports leagues, the sports business as a whole is lagging behind other industries in their use of data, as Sascha Schmidt puts it: "With all the excitement for sports, however, we cannot neglect that, from a business perspective, sport is one of the most conservative industries on the planet'.

The use of data in strength training ( $\mathrm{S} \& \mathrm{C})$ is has been very limited, partly due to the lack of suitable measurement technologies (see figure 1 later) that would allow continuous high-quality measurement without being too cumbersome for the athlete [882]. Nowadays, the strength training protocols are based on practical/clinical experience and evidence-based approaches with current evidence however, being sparse. There is a need for further advancement towards more more quantitative strength training frameworks integrating objective physiological measures with subjective measures, going beyond one-size-fits-all models. This framework introduced in this review is referred as precision strength training, inspired by recent advances in precision medicine.

Precision medicine, sometimes referred as personalised medicine, aims to quantitatively model the intra- and inter-individual variability of patients in response to treatment [152] (see figure 1). 
The concept of personalising care to the patient is not new, but recent advances in large scale databases, better measurement technologies, and computational tools, such as deep learning, are making data-driven precision medicine realistically achievable [432]. Data-driven precision medicine however has not yet become the clinical norm, requiring more clinical validation and model development for precision medicine to become one $[15,380,761,873]$. The use of precision medicine framework for exercise prescription and monitoring is not new either. Buford et al. [99] described an 'exercise as medicine' framework for general population, and Ross et al. [690] demonstrated a model that captured inter-individual differences in response to cardiorespiratory exercise, coining the term precision exercise medicine. Precision health is conceptually close to precision exercise, as it attempts not just to address the symptoms, but to promote and prevent diseases on a population and public health level [253, 313].

Early precision exercise medicine literature was focused mainly on quantifying inter-individual (between subjects) differences in intervention response and identifying responders/non-responders to exercise [36], with less work devoted on the role of intra-individual (within-subject) variations. This inter-individual focus was shown to erroneously suggest large inter-individual variation when not addressed properly in the experimental designs and statistical analysis [36, 144, 194]. In a study by Islam et al. [362], the athletes showed significant intra-individual variability to the same training stimulus, and the authors highlighted the fact that without the use of repeated trials, some of the subjects could have been wrongly classified as high or low responders. In contrast to traditionally used net mean treatment effect in studies, one is interested in the individual responses [600] within precision exercise framework, and how measurement noise (that is random) can be disentangled from the inherent physiological response (that is reproducible) [361]. While there have been advances in statistical methods to analyse these heterogeneous responses [174, 801], the future studies need to consider jointly the research protocol design, measurement technologies, and modelling tools for disentanglement of sources and causal relationships [761, 776, 836].

In strength training, concept of personalised training (referred also as autoregulation [276]) has existed for some time, in which the training load and recovery status of an individual athlete are continuously monitored, and the exercise continuously adapted by a human coach (subjective decision making) using the monitored parameters output [61, 135, 537]. The scientific challenge in practice with this framework has been the proper parametrisation of 1) the training load $[40,55,654,883] ; 2)$ the recovery state and preparedness to train [322, 394, 537]; 3) injury risk prediction [356, 357, 601, 755, 881]. Out of these three goals, training load and recovery state are within practical reach at current technological maturity level, whereas injury prediction seems overly challenging $[315,356,357,385,386,599,678]^{1}$, mainly due to inherent low prevalence of injuries in athletic population [101, 105, 344, 377, 397, $627,686,743,878]$, which subsequently leads to injury prediction models with poor predictive power [348, 504].

In precision medicine literature, personalised and precision medicine are sometimes used interchangeably, where in this review the "precision' in precision strength training framework refers to the dynamic data-driven training program individualised for each athlete, and updated dynamically based on the 'precision biopsychosocial model'. The definition of autoregulation (personalised training) framework is relatively ambiguous in the literature [276], and the approach described in this review fits to existing loose definition, but we wish to stress the quantitative modeling aspect with our precision prefix, inspired by the precision medicine literature. In brief, precision medicine is interested in developing disease progression models [902], prescriptive modeling with individualised treatment effects [78, 141, 473, 625], phenotyping patients [593, 832, 866], and acquiring patient similarity measures [729].

Transferring these tasks to the strength training context (see figure 1), the training progress can be interpreted as the disease progression trajectory, the changes in training program and active recovery interventions as medical intervention/individualised medical treatments, and the clustering/phenotyping athletes based on their response to training, e.g. machine learning recommender system for sports [254, 574] with recommendations like you "athletes with similar trunk:thigh ratios cannot squat as upright'.

The personalisation in strength training, is typically based on subjective measures for daily preparedness and logged training loads, with training adjustments done subjectively by either human expert [210, 276, 392, 875], or by non-learning mathematical methods [104, 231, 328, 329, 343, 597, 749]. A practical commercial example of the mathematical modelling approach for strength training is the JuggernautAI ${ }^{\circledR}$ system (https://www.jtsstrength.com/product/powerliftinga-i/), which first surveys basic athlete characteristics, then designs training periodisation and updates the program dynamically based on the logged progress of the athlete. This type of system can be seen as an upgraded training diary/logger with more advanced training

${ }^{1}$ Sam Robertson's tweet, Aug 2, 2019 twitter.com/robertson_sj/status/1157188689702707200 
feedback [509, 804], examples of traditional loggers are Gravitus ${ }^{\circledR}$ (https://gravitus.com/), Strong ${ }^{\circledR}$ (https://www.strong.app/), gymaholic ${ }^{\circledR}$ (http://www.gymaholic.me/). These consumerlevel train loggers do not typically handle aggregation of objective measures (see section §III later), and their use for advanced data-driven training modeling is somewhat limited.

In larger professional sports organisations, more advanced athlete management systems (AMS) [268, 541] are used instead these consumer-level training diaries. For example, Smartabase ${ }^{\circledR}$, an AMS developed by Fusion Sport ${ }^{\circledR}$ (https://www.fusionsport.com) integrates electrical medical records (EMR) [789] and athlete performance data with the possibility to develop multimodal models while managing of multiple athletes. Such systems are used by various professional sporting organisations ranging from UFC ${ }^{\circledR}$ Performance Institute [471] to The Royal Ballet (UK) [180]. At present, athlete management systems lack interoperability application programming interface (API) standards [642] such as the one developed for healthcare, like the HL7 FHIR version 4.0.1 introduced in USA starting from 1st January 2021 [568, 705, 812]. Many vendors such as Kinduct ${ }^{\circledR}$, Smartabase ${ }^{\circledR}$, Edge10 ${ }^{\circledR}$, Kitman ${ }^{\circledR}$ and BridgeAthletic ${ }^{\circledR}$ have though formed partnership allowing cross-vendor aggregation of athlete data ${ }^{2}$.

Precision strength training is similar to precision physiotherapy in which the physical rehabilitation program (e.g. after an orthopaedic surgery or a stroke) is being individualised to the patient with data-driven deep learning models and gamified therapy [86, 463, 496, 785, 885]. In United Kingdom an interest group called "Digital and Informatics Physiotherapy Group (DIPG)" (part of the Chartered Society of Physiotherapists, CSP) was formed to develop, evaluate and promote was formed to promote the use of novel technologies such as virtual reality (VR), telerehabilitation and AI in clinical physiotherapy. Having possibility of patients exercising at their homes with automatic real-time exercise feedback [161, 173, 209, $294,477,538,595,674,675,704,815]$ could positively influence exercise adherence leading to improved patient outcomes and lowered healthcare costs [445, 884]. Similarly, these personalised and gamified approaches could be designed for general population as a preventive health measure [825], for example to increase the uptake of resistance training [63, 867], as $80 \%$ of European adults do not meet the global resistance training guidelines of 2 or more days of resistance training per week [62].

\footnotetext{
${ }^{2}$ https://blog.bridgeathletic.com/integrating-your-
}

As precision strength training does not yet exist as an established field, we derive the concepts in this review from literature on evidence-based personalised strength training[562], precision physical rehabilitation [885], deep learning [873] and sport science [126, 653, 677], to give the readers an overview of plausible future scenarios in strength training. We will review the challenges involved in the development of precision strength training framework by going through the relevant sports measurement technology, dataset requirements, strength training theory and service design for relevant for deploying the system to be used by the patient, clinicians, athletes and their coaches, in an effort to try to bridge the gap between in-lab theoretical sports science and real-world deployment [97, 529, 531, 865].

In this review, we refer to three generations of strength training systems: 1) tradition-based training, that is often referred as practical or clinical experience, and is the tacit knowledge gained by the practitioners; 2) evidence-based training, that is based on scientific strength training studies with relatively narrow inclusion criteria applicable to specific populations and set of assumptions. The intra- and inter-individual variability is mitigated by selecting relatively homogeneous populations; 3) data-driven precision strength training, that uses broader inclusion criteria and attempts to model the exercise responses of a larger heterogeneous population. The intra- and inter-individual variability in response to training is learned from the acquired using multivariate (high-dimensional) AI models.

We will envision strength training and sports management to follow the advances in medicine and society as a whole, with the trend for increased human-machine interplay in human-inthe-loop systems, that are developed augmenting human experts rather than by replacing them with $\mathrm{AI}[49,258,279,310,615,742,753]$. It is not trivial to transfer and quantify the tacit knowledge from athlete-coach to be used for quantitative modelling [81, 482, 588], and the " $n=1$ " expert knowledge need to built into these precision strength training frameworks [332, 442, 478, 566, 634, 722, 740, 831]. Eventually, over the coming decades, the more conservative "non-tech" strength training coaches will be re replaced by the strength training coaches embracing AI, as it is projected to take happen for example to radiologists [721].

The review has been written with a focus on machine learning practitioners, who are familiar with mathematical modeling concepts, and less with strength training and sports science domain knowledge [616, 677]. We take a systems-level approach in this review [33, 64], covering measurement technology, basic modeling concepts, dataset requirements and user experience in future quantitative strength training systems [145, 850], built on top of the evidence-based strength training re- 
search [563]. Less emphasis is placed on the recent emerging exercise physiology concepts such as "network physiology of exercise' that is a systemlevel approach on how physiological states emerge from complex nonlinear interactions within human body [44]. In general, we would hope that this review is a good starting point for data scientists, entrepreneurs and sports scientists understanding the main challenges in strength training modelling, as often in digital health, solutions are developed for non-existing problems [483, 525].

\section{FROM PERSONALISED TO PRECISION STRENGTH TRAINING}

In this review, the term personalised strength training refers to the current evidence-based strength training practice [562], where athlete are being monitored with some sensor technology, but majority of the training intervention decisions are being made by a human expert (e.g. a coach or a physiotherapist). The existing quantitative frameworks, for example for injury prevention and prediction, are not the most useful at the moment for expert-level coaching [356]. In practice, there are no quantitative frameworks augmenting coaching decision, and human coaches are often overwhelmed by the various training load and recovery metrics, and often fall back on more conservative evidence-based or practical experience measures [406, 601]. This is especially true in strength sports such as in powerlifting, in which the practitioners often resort to trial and error approaches, as evidence-based protocols are lacking [821]. None of the suboptimal quantitative frameworks, such as acute:chronic workload ratio (ACWR) [20, 851], are truly data-driven and are rather more conceptual models. There have not been any major artificial intelligence studies to the authors' knowledge for strength training research, with the few studies using machine learning have used small datasets mostly for proof of concept studies, and not for real-world deployment [653].

Strength training (or $\mathrm{S} \& \mathrm{C}$ ) can be broadly defined by three types of training goals: 1) maximal strength production (e.g. powerlifting) [563, 912]; 2) hypertrophy training to increase muscle mass (e.g. bodybuilding) [364, 379, 716, 750]; 3) rate of force development (RFD), the production of explosive strength for example in Olympic weightlifitng, boxing and track and field sprinting [197, 603, 717, 829]. In practice, a mixture of the goals are present, especially in non-strength sports, in which for example both muscle mass and increased maximal strength might be desired (e.g. American football), and in weight-class sports such as boxing, explosive strength might be desired without the added muscle mass. In these cases, the monitored metrics should be adjusted accordingly to the specific sports in question [308, 322].
The need of sports-specific measure is highlighted for example by the possible counterintuitive effects of hypertrophy to strength levels described in recent research. Reggiani et al. [664] showed that the increase in muscle mass did not necessarily lead to increase in strength and vice versa [664], and in worst case, the increase in muscle mass was shown to decrease strength production [664, 718]. There also seems to exist multiple types of muscle hypertrophy [664, 820], with the concept of task-specific hypertrophy [820] becoming relevant when trying to transfer the gains from strength training program to improvements in the particular sport of the athlete [906]. For instance sports as different as combat sports [485, 765] and ballet [16, 180, 818], can be benefited for similar plyometric/ballistic training for explosive force production [906] mixed with some maximum strength training. Whereas in sports like pole dancing that do not require explosive strength, benefit more from powerlifting-type maximum strength training [874]. In circus training [293, 435] and in throwing sports [82, 340], more emphasis should be put on warming tendons and ligaments properly, and making sure that the tendons adapt to training and do not fall behind from faster muscle strength adaptation [387].

One-repetition maximum (1RM) is probably the most commonly used tool at the moment to personalise strength training programs [810]. Athlete's training loads are programmed using some percentage of 1RM, e.g. at week 1: 3 sets of 5 repetitions at $60 \%$ of $1 \mathrm{RM}$, week 2 : same at $70 \%$ of $1 \mathrm{RM}$, and so on. These percentage progressions are typically programmed using some traditionbased values, that the practitioners have found effective in the past, using training cycles of various lengths. This splitting of training to cycles is referred as periodisation often involving the following concepts: macrocycle (e.g., 1 year between the main competition of the athlete), mesocycle (e.g., 4 weeks), microcycle (e.g., 1 week) and individual training sessions. For example in powerlifting, the goal of macrocycle, is to increase the 1RM of the athlete as much as possible, while monitoring the progression of 1RM during that macrocycle $[40,763,819]$. In practice, proxy measures need to be developed for this progression monitoring, as the athletes are not often programmed to lift $100 \%$ of 1 RM very often, as such heavy loads during training season are seen counterproductive for the overall progress and needlessly increasing the risk of injury. Thus, one wants to find a balance between of not overtesting the athletes, without the test measure possibly coming the goal itself [40, 699], and not undertesting the athletes and not without being able to track the training progress [819].

Research has been devoted for finding indirect (proxy) measures for the 1RM progression, with no conclusive measure for strength training. For 
A

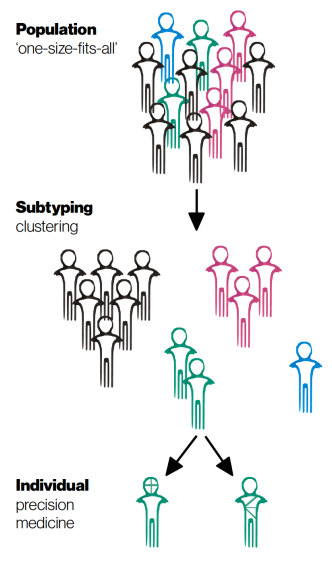

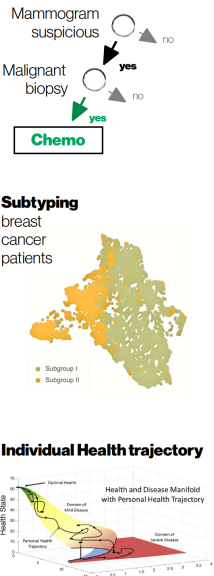

B

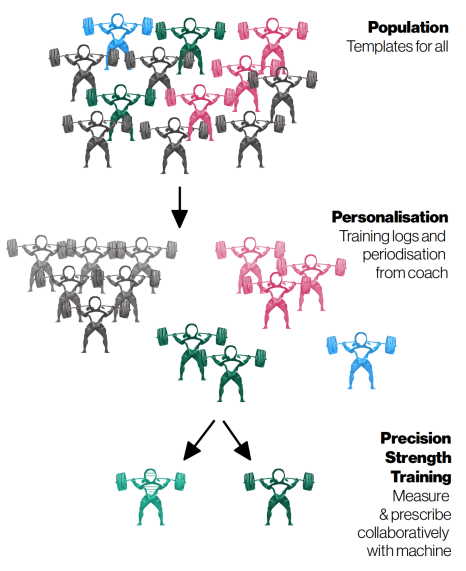

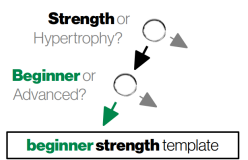
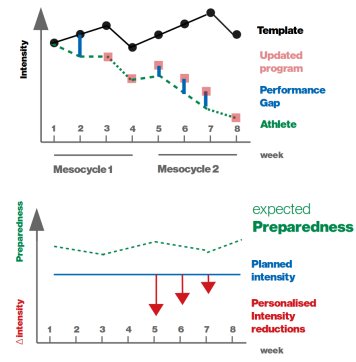

Figure 1. Precision medicine vs. precision strength training. (A) Simplified model of traditional 'one-sizefits-all' medicine uses simple decision rules for treating all patients the same [168], whereas precision medicine attempts to tailor the treatment based on different subtypes [455] or individualise the treatment based on the individual patient [181]. The health states on the three-dimensional trajectory manifold in strength training can be interpreted as progress, the athlete needs to have the peak performance (health state) in competition, and optimal decisions need to be taken to get there. Some decisions on the manifold takes the athlete only sideways, i.e. performance is plateauing despite the modifications to training. Note that both subtyping and health trajectory are visualised with dimensionality reduction techniques such as t-SNE or UMAP to make highdimensional nonlinear models human interpretable [43, 423, 517, 782]. (B) In strength training, the equivalent to one-size-fits-all approach are the training program templates offered often online with little customisation to the athlete [443]. Personalised strength training (or autoregulation) is often done with a coach who updates the program for the athlete [169]. In our simplified example, two training mesocycles are shown, in which the athlete is able to do the programmed exercises on week 1 but fails to do so on week 2. On week 3 the programmed loads are dropped to the levels of week 2 [321], and the athlete is able to keep up with the programming during the first mesocycle. During second mesocycle, the athlete shows overtraining syndrome, and keep up with any of the programmed loads ("performance gap'), except on the week 4 that is a deloading week. In our simplified precision strength training framework, the level of training preparedness would be quantified, and overtraining symptoms could be reduced faster by reducing training loads (personalised intensity reductions) when the model has detected that the athlete has not recovered properly. The accuracy of such automatic adjusting obviously depends on the quality and availability of data for training such models as we review in this article.

example squat jump height (SJH) [819] and isometric mid-thigh pull (IMTP) [374] have been used as proxies for Olympic weightlifting monitoring; multiple repetition test [671], and mean concentric velocity (MCV) from load-velocity curve have been used for 1RM estimation in powerlifting [50, 862, 876]. Common problems with these testing measures is that the testing itself is too physically demanding requiring a recovery period itself [667], and that the athletes might become better in doing the proxy measures themselves during the monitoring period, i.e. there is a learning effect occurring, that can bias the measurements and increase the uncertainty in progression monitoring [763].

Conceptually, a more complete approach for performance tracking, is to use for example the acute:chronic workload ratio (ACWR) [358], that is based on classical fitness-fatigue model [106]. In ACWR there are internal and external loads [297, 355-357]. The external load is the prescribed exercise session, e.g., the training load and volume. The internal load corresponding to the training preparedness and recovery state, including all the factors influencing athlete's ability to recover, such as sleep quality [66, 136, 287, 480, 745, 844, 847], nutrition $[26,85,112,554,558,614,750,826]$, and overall stress in life [324, 365, 771], i.e. constituting for the biopsychosocial model of the athlete $[319,809]$. The external load is easier to quantify with less uncertainty, assuming that the athlete is doing every workout with repeatable technique and focus ("exercise adherence") [810, 926]. The ACWR as a quantitative model however does not seem to perform at a satisfactory level, and in practice expert judgement is preferred over its predictions [356], thus the key idea of the precision strength training framework is the development of a quantitative biopsychosocial model that would help to explain the observed intra- and inter-individual variability responses to training programming [280].

There is an ongoing transition from traditionbased strength training, so-called 'bro science' in gym jargon [2], to evidence-based strength training [563, 716], with some strength \& coaching coaches resisting this cultural change [2]. Some scepticism to evidence-based training is warranted, as 
A Personalised Evidence-based

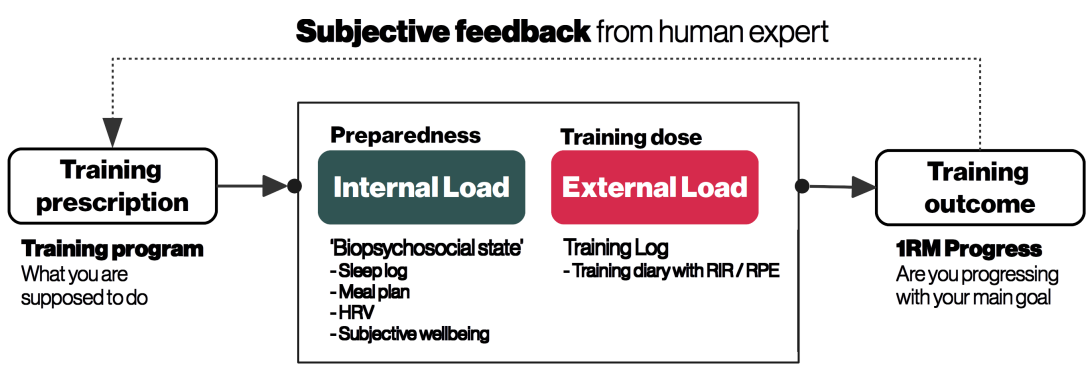

B Precision Data-driven

Semi-objective feedback with human-in-the loop with machine

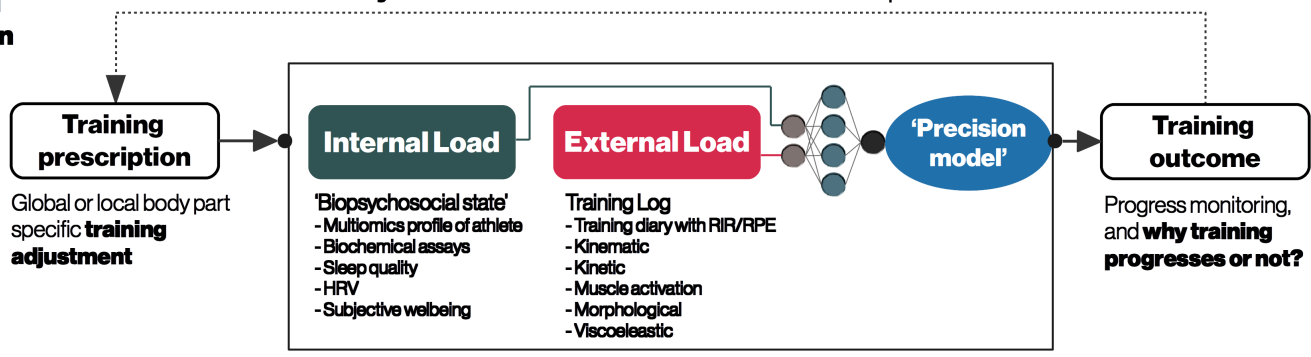

Figure 2. (A) Personalised (evidence-based) strength training [276, 563, 629] in which the human coach interprets the process and makes the decision for the athlete being guided by science [2], using some tradition-based heuristics [169]. Some of the training is quantified for example in a form of training log with subjective rating of perceived effort (RPE) or reps in reserve (RIR, and mathematical models can be developed from them. The dose-response to training prescription depends on the athlete's internal load [280, 324, 327, 355, 507], that is not often captured quantitatively by the existing frameworks such as acute:chronic workload ration (ACWR) [357]. (B) In idealised future, the precision strength training framework built top on evidence-based training is able to quantitatively measure and model both the internal and external load and adjust the training and recovery activities accordingly collaboratively with the coach.

several gaps between the theory and practice in strength training research exist due to the field being so small, and therefore, practical coaching decision thus often requiring a lot of improvisation around the fragmented evidence. Evidencebased medicine (EBM) has faced similar resistance to change from its inception in the early $90 \mathrm{~s}$ [89, 221, 421, 514, 548], with some clinicians stating that EBM has led to diminished acceptance of "the art of diagnostics" or personal clinical judgement [275, 454].

Similar resistance to change is felt with the recent early implementations of precision medicine [15, 696], both at the organisational level [696] and at the scientific level in terms of implementation readiness. For example, molecular profiling, clinical risk modelling and pharmacogenomics ( $\mathrm{PGx}$ ) are more mature and closer to broad implementation compared to less understood genomic EHRs and disease subtyping [15]. The overselling of model such as ACWR [356], can have its analogy in computer-aided detection (CAD) for mammography in breast screen screening. The CAD models were constantly underperforming compared to human readers [304], leading to an increased cost of proofreading and evaluating unnecessary falsepositive results [211], and eventual distrust of all algorithmic solutions. Recently, Ziegelstein coined the term personome in 2015 to describe the process of bringing back the person of the patient, and how the patient biopsychosocial state with quantitative measures modulate their response to treatment [931]. This is similar to our vision on how in precision strength training framework, the full biopsychosocial model of the athlete is formulated in more quantitative framework.

\section{MEASUREMENT TECHNOLOGY FOR PRECISION STRENGTH TRAINING}

We will review here the most commonly used measurement technologies for monitoring athlete's strength performance.. The cost and size of highend motion capture systems have traditionally constrained the use of these technologies in field studies and outside the sports science laboratories. With emergence consumer devices such as Microsoft ${ }^{\circledR}$ Kinect $^{\mathrm{TM}}$ re-purposed for sports science, the cost of technology has become less of an issue [14]. Recently interesting developments in wearable compressive garment-based sensor platforms [286] and novel sensors/actuators developed for extended reality (XR) [383, 854] and soft robots applications [738] are enabling the design of future non-invasive and cost-effective continuous performance monitoring solutions for strength training.

The development in sensor technology and its ease of use might refine the definitions of the test- 
ing (done infrequently) vs monitoring (done often) variables used by some $\mathrm{S} \& \mathrm{C}$ coaches [667]. Some of the measures such as blood sampling and dual-energy X-ray absorptiometry (DXA) currently seen as testing measures might become monitoring measures with the introduction of lowcost and accurate future alternatives [252, 303, 333, 456, 462, 633, 814]. Some functional test measurements such as isometric mid-thigh pull (IMTP), might be replaced with something less physically demanding to make their use more frequent as a monitoring measure. While often in functional testing, it is the simplest to use some of the main exercise movements, such as bench press or squat, at the end of each mesocycle for progression monitoring, without increasing fatigue levels of the athlete [363].

Similarly, the more athlete-friendly sensor technology could make monitoring more time-efficient and better integrated to training routines. This especially applies in team sports in which athletelevel monitoring is sometimes challenging [766]. Future studies should also look into developing a proper "sports economics' frameworks, inspired by health economics $[42,127,151,569]$, which would include the time and financial cost of given measurement technology compared to its expected value for athletic performance.

Easily acquired continuous measurements are not always helpful when the signal has low fidelity, or when the data is not the most relevant for the task in question [113]. Example of consumer-level large-scale data acquisition can be found from the "quantified self" (QS) movement, in which participants track their biomarkers as biohackers for data-enabled self-improvement $[4,175,219,528,811]$. Participants in QS movement do not always feel the measurement as source of motivation and entertainment as often portrayed [163], but rather feel anxiety from selfmeasurement and not being able to meet their goals $[18,52,219]$. Similar patient anxiety has been shown with recent digital health solutions, such as implementation of atrial fibrillation detection via smartwatches, that do not seem to offer high enough signal fidelity, leading to excessive false-positive diagnoses and incurring needless expenses and patient anxiety [621]. In contrast, continuous data acquisition with smartwatches seem to be useful for monitoring Parkinson's disease patients[637], helping the clinicians to track the symptoms and manage treatment better [8]. In practice, even at the highest signal quality, the measured variables cannot capture the athlete's whole biopsychosocial performance. Therefore, the inherent uncertainty in the combined measures should be taken into account when using the measures for decision-making [40, 299, 684], to avoid key performance indicator (KPI) fixation like ones seen in university evaluation systems [773].

Most of the recent products from digital thera- peutic and sports analysis companies such as Kaia Health $^{\circledR}$ (https://www.kaiahealth.com/), Fitcus ${ }^{\circledR}$ (https://www.fitcus.com/), Curv Health ${ }^{\circledR}$ (https://www.curvhealth.com/), and Kinetisense ${ }^{\circledR}$ (https://kinetisense.com/) rely on unimodal kinematic video data, either using a standard smartphone camera or by the company-produced custom camera. Whereas companies such as Figur $8^{\circledR}$ (https://figur8tech.com/) has chosen to complement their inertial measurement unit (IMU)-based kinematic data with muscle activation data from a mechanomyographic (MMG) sensor, for a multimodal measurement of the athlete behaviour. These business choices have been partly driven by consumer expectations of a delivery via smartphone apps, and the longer development times associated with new hardware development favouring software solutions over hardware solutions [68]. We hypothesise that smartphone-based kinematic data might not be of sufficient quality for training precision strength models, to obtain good enough actionable insights [754, 930], to surpass or augmenting well the human expert opinion. However, eventually with large enough multimodal datasets acquired, precision strength training models could be trained with multimodal datasets, and deployed then to be used only with video data, and to approximate the missing modalities such as kinetic data, from the unimodal kinematic data alone [139, 250, 376, 657].

\section{A. Kinematic: Motion Capture and Pose Estimation}

Motion capture (mocap, mo-cap) refers to the quantification of movement patterns of objects (e.g surgical tools), animals, and people [58, 527, 712, 796]. In sports and rehabilitation applications, the study of these biomechanical measurements are often referred as movement science [269, 769]. The estimated human pose from video or sensor stream [923], is typically encoded as a skeleton pose sequence that is a time series of three-dimensional joint locations [298, 444, 493] (see figure 3). The technical details on how video, sensor, radiofrequency (RF) or point cloud data is transformed into skeleton sequences is beyond the scope of this review, and the interested readers are referred to following works [345, 372, 617, 645, 898].

The choice of the deep learning approach for modelling skeleton sequences, is typically based on graph convolutional networks (GCNs) [736, 916], where the nodes of the graph represent the 3D joint locations, and edges of the graph represent the bone connecting the joints. In addition to this basic joint-level encoding, one can encode additional features on the graph (if acquired) on the edges and nodes of the graph [492, 632, 736]. For example muscle stiffness and muscle activation are useful in strength training and can be encoded on 


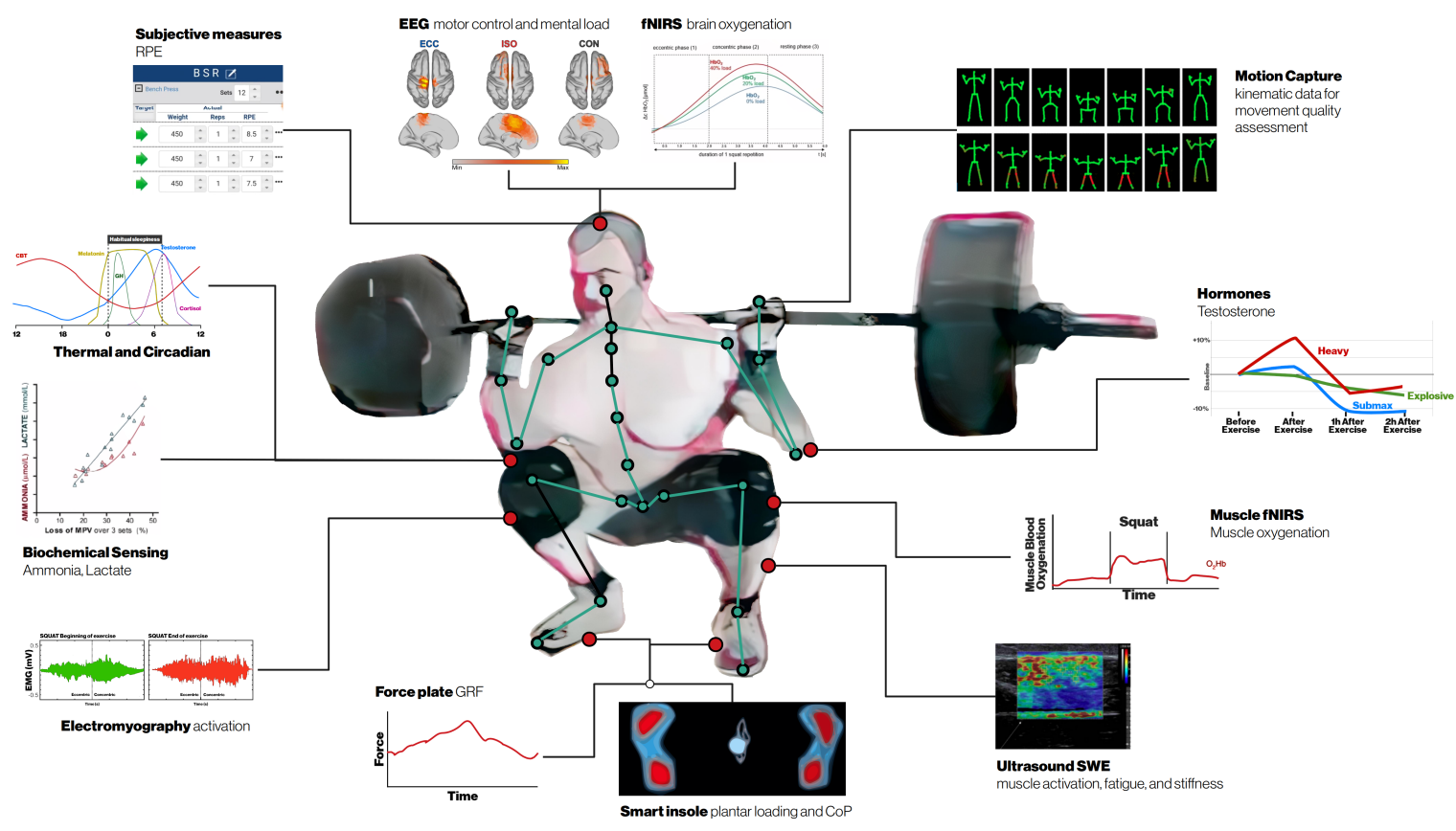

Figure 3. Example set of measurement modalities useful for developing precision strength training frameworks. Motion capture (kinematic) data for movement quality assessment [607], testosterone assay for both acute exercise-induced response and global levels [288, 484], muscle oxygenation with fNIRS [679], ultrasound SWE for the (real-time) quantification of musculotendon stiffness [419, 467], cartoon of smart insoles for quantifying center-of-pressure (CoP) and the tripod loading, force plates for measuring ground reaction forces [245], EMG for measuring "activation" [257] ("myoelectric activity" more exactly [841]), sweat-based biochemical sensing for detecting metabolic and neuromuscular fatigue from ammonia and lactate levels [697], core body temperature for circadian phase [788] and muscle temperature measurements for training preparedness, subjective rating of effort through RPE logging (https://www.reactivetrainingsystems.com/), cognitive load from electrical (EEG) [396] and hemodynamic (fNIRS) brain activity [395]. Image from Dr. Ben Pollock, reproduced with permission. fNIRS functional near-infrared spectroscopy, SWE shear-wave elastography, RPE rating of perceived effort

the edge of the graph; tendon stiffness and range of motion (ROM) can be for example encoded on the node of the graph.

The GCN formulation also allows to use traditional signal processing techniques such as dynamical mode decomposition (DMD) and Koopman operator [503, 561, 892] to approximate nonlinear human locomotion with a computationally simpler linear system [247]. However, recently neural ordinary differential equation (ODE) based models have shown to be a more versatile approach compared to DMD, with better intrinsic handling of missing and irregularly sampled data [132, 780, 901]. Additionally, graphs (single athlete) can be embedded into larger graphs (e.g. team of athletes in a basketball game), if one would be interested in how group dynamics (e.g. basketball game) affect the individual biomechanics [248], or vice versa.

There are a variety if different motion capturing technologies including low-cost inertial measurement units (IMUs) [619, 680], wearable cameras ('egocentric pose estimation') [179, 350, 489, 502], smartphone cameras [497, 539, 643], Kinect ${ }^{\mathrm{TM}}$ type depth cameras [14, 102, 148, 626], and highend motion captures system such as Vicon ${ }^{\mathrm{TM}}$ or Optitrack ${ }^{\mathrm{TM}}$, with the prices of high-end system reaching up to to $\$ 200,000$ [808, 817]. Different technologies offer different spatial and temporal resolutions, and make different tradeoffs in terms of performance and cost. For example the spatial accuracy of joint locations is especially relevant for tracking very subtle changes in movement patterns [575, 728], and high temporal resolution is desired for fast-paced sports such as mixed martial arts (MMA) [907]. The choice of used technology ultimately depends on the application, but for our strength training application in advanced athlete population, we are more interested in spatial resolution than temporal resolution. As highlighted by Seethapathi et al. [728] for movement science research, the off-the-shelf deep learning models such as OpenPose [109] trained on generic movement datasets such as NTU-RGB 120 [486] might not provide good enough spatial joint locations highlighting the need for custom dataset acquisition (see IV C below).

The video-based motion capture systems offer a higher spatial accuracy compared to low-cost IMUs, that are based on sensing changes in gravity (accelerometer) and rotation (gyroscope) [680], and converting those measures into estimates of human movement. IMUs offer higher temporal precision at lower cost compared to cameras. Ad- 
ditionally in contrast to cameras, the IMUs can be worn by the athletes as a smart compressive garment [411, 893], developed for example by companies such as Xsens ${ }^{\mathrm{TM}}$ (https://www.xsens.com/), Cape Bionics ${ }^{\mathrm{TM}}$ (https://www.capebionics.com/) and Teslasuit ${ }^{\mathrm{TM}}$ https://teslasuit.io/. The wearable compressive garment is relatively invisible to the athlete, but the technology has not gained the market traction as hoped by the companies and practitioners [155]. IMU-based systems additionally can be used for continuous measurement [663] when longitudinal trends and day-today fluctuations in movement patterns are of interest [172]. The challenges with the use of IMUs is their time-dependent drift [868], soft tissue artifacts due to skin motion in relation to underlying bone [236, 460, 909], and the need for complicated calibration making their use cumbersome [71, 803, 868]. Recently, low-cost alternatives to IMUs have been developed, such as strain sensors embedded in knee braces [262], and as electronic skin sensors for monitoring lumbar-pelvic movements [919].

\section{B. Kinetic: Force measurement}

The kinematic data only quantifies the biomechanics of athlete performance, e..g the squat movement pattern [607] but does not tell anything about the forces produced by the athlete, for which we need to measure the kinetic data [413]. Multiple technology options exist for kinetic data measurement including low-cost smart insoles, Nintendo Wii Balance Board, and highend "gold standard force plates for kinetic data, that can be used to derive ground reaction forces (GRFs), rate of force development (RFD) and centre of pressure $(\mathrm{CoP})$ parameters. In sports performance laboratories, the force plates are routinely used [526, 759], but recently low-cost insoles [195, 250, 378, 612, 795, 864] and Nintendo ${ }^{\text {(B) }}$ Wii Balance Board ${ }^{\mathrm{TM}}$ [543] have been investigated as alternatives as the cost of force plates can be prohibitive in some cases. Recently, nonlearning mathematical [863], and deep learning models have been developed to approximate the forces generated by the athlete, solely from nonkinetic measurements, such as from kinematic data [323, 375, 724]. These approaches naturally require development datasets with the both modalities (kinematic and kinetic) in order to validate the approximation accuracy of the model when deploying to real-world use just with the kinematic modality.

\section{Muscle activation measurement}

In addition to the kinematic and kinetic data, strength training research is often interested in how and when muscle is activated/recruited during movement [261], when muscle experiences fatigue [513], and how different neuromuscular processes are involved in force production [415]. The most common measurement technology used for these purposes is surface electromyography (sEMG) [6, 73, 100, 115, 784, 841], that involves placing electrodes on top of the skin, either as individual single sensors or embedded onto a wearable smart compressive garment suit [189, 286, 411, 752, 896]. Kim et al. [411] studied the effect of garment fit, i.e. clothing pressure, to sEMG signal fidelity, and showed higher pressure led to improved signal-to-noise ratio (SNR) highlighting the need for customisation of smart garment. This has been applied in practice, for example by Cape Bionics $^{\mathrm{TM}}$ company, scanning the athletes in 3D (see e.g. [814]) and customising the suit from the scan to the given athlete.

There has been some controversy on the usefulness of EMG in strength training research [159, 216, 840, 841], with some people claiming EMG being totally useless for strength training [159], while some have argued EMG having still value given proper interpretation knowing the limitations [840, 841]. It has been acknowledged that one cannot infer from the EMG amplitude alone the number of the motor units recruited to complete a specific movement/task because the number will exclusively depend on the muscle group(s) being recorded [257]. Mesin et al. [544] for example showed that tibialis anterior has a superficial-todeep recruitment pattern, causing the sEMG amplitude to rise faster than the force levels. This suggests that in some cases, both surface and intramuscular electrodes could be used simultaneously [924], for more complete view of the muscle activity. The use of sEMG seems always justified for studying the timing characteristics of muscle activation and deactivation, information that could be used in musculoskeletal model simulations [464].

Vigotsky et al. [841] argue that one thus should avoid using the term "muscle activation" when referring to EMG amplitudes, and utilise terms such as 'myoelectric activity' or 'muscle excitation', as sEMG measures changes in muscle fibers' membrane potential. Throughout this review, we use the more generic term 'activation' in broad sense for all the techniques aimed for measuring some sort of muscle activation/excitation. At the moment there seems to be no evidence suggesting that sEMG is suggestive of hypertrophy monitoring [296], nor there is really evidence to suggest that training load recommendations could be extrapolated from sEMG amplitudes [841]. More studies are needed to understand the training load 
programming and sEMG amplitudes [841]. Detailed review of sEMG intricacies is beyond the scope of this article, and interested readers are referred to the excellent review by Vigotsky et al. [841].

Furthermore, it is also possible to to use the intramuscular or surface electrodes to drive muscle activity, known as electromyostimulation (EMS) [229], neuromuscular electrical stimulation (NMES) [237], functional electrical stimulation [399], electrical muscle stimulation [220], or electrical dry needling [199]. In strength training, electrical stimulation can also be used to test the force production of muscles such as $m$. abductor hallucis $(\mathrm{AbH})$ that are often hard to voluntarily activate [592]. Recently, sEMG research and technology development have been driven by humancomputer interaction (HCI) field with the interest for hand gesture sensing [577, 854], from companies such as Facebook ${ }^{\circledR}$ that recently had acquired companies CTRL-Labs ${ }^{\text {TMTM }}$, a company who owned the intellectual property (IP) for the $\mathrm{Myo}^{\mathrm{TM}}$ EMG armband also used in exercise applications [431, 453, 479, 556].

Complimentary to electrophysiological sEMG, there are its "mechanical counterparts': mechanomyography (MMG) [790], force myography (FMG) [59, 891] and tensiomyography (TMG) [494]. They are similarly measured from the surface of the skin, while not commonly used in sports application, when done, they are acquired simultaneously with sEMG. Sonomyography (SMG) is an ultrasound-based "morphological counterpart' of sEMG [285, 692], and is also often measured simultaneously with other activation modalities for complementary activation information. Functional near-infrared spectroscopy (fNIRS) [535, 701] can be used to measure the hemodynamic muscle response to study muscle oxygenation behaviour [23]. Similarly to other complimentary methods, it is often used simultaneously with sEMG as recently demonstrated in a wearable suit designed for European Space Agency (ESA) space crews [188].

\section{Muscle morphology and its viscoelastic properties}

In addition to the kinematic, force and activation data, researchers are often interested in the morphology of the musculoskeletal tissues, i.e. its geometry, thickness, and stiffness. Ultrasound imaging (ultrasonography) is the most commonly used technology for measurement of muscle properties, due to its high spatial resolution and relatively low cost [813]. Ultrasonography can be augmented with ultrasound shear wave elastography (SWE) that enable continuous measurement of viscoelastic properties of musculoskeletal tissues [166]. SWE can be used to study the muscle stiff- ness after training session [830], Achilles tendon stiffness in natural environments using a wearable SWE [302], Achilles tendon stiffness and its effect on ROM in squat [270], dynamical muscle contraction during squat [571] with deep learning inversion [224], changes in viscoelastic properties of muscle during fatigue [119], and efficacy of stretching program [21, 240]. Myotonometry (MMT) is a low-cost alternative to SWE for assessing musculoskeletal tissue stiffness, that was recently used simultaneously with SWE to construct a 3D spatial map of muscle stiffness [419], and with TMG to measure neuromuscular response and muscle stiffness [452].

With the acquisition of kinematic, kinetic, activation and morphological parameters of an individual athlete, it is possible to construct personalised neuromusculoskeletal (NMS) models [186, 227, 407, 446, 630, 709]. Moreover, with the continuous acquisition of athlete-specific parameters, personalised dynamic neuromuscular model for real-time feedback and visualisation are possible to develop with deep reinforcement learning (DRL) as done recently by Lee at al. [464]). Same model can be used then to track the longitudinal musculoskeletal changes over a training cycle, that could be indicative of training progression, injury risk or overtraining. Typically, the translation of such models to field use or typical clinical applications is challenging as often the acquisition of all these modalities are not done. There is a desire to either sample the missing modalities from a generative deep learning model [177, 737, 889], or train a deep learning model to approximate a full set of measures, using for example a low-cost wearable sensors such as IMUs [630].

\section{E. Overtraining: Heart Rate Variability (HRV)}

Heart rate (HR) monitoring, either via optical photoplethysmography (PPG, e.g. on ring or on smartwatch) or electrocardiocardiographic (ECG) chest straps, are probably the most commonly used wearable sports technology. HR monitors are frequently used in endurance sports monitoring [838], but they have been recently used in strength training to assess the overtraining/recovery status of the athlete [511, 655, 805], often involving overnight sleep recordings rather than during the training session itself $[756,877]$. From these measurements, heart rate variability (HRV) is the most commonly derived parameter [193, 389], with the ring or wrist band monitor/sensor offering ease-ofuse and good adherence. However, the relatively lower signal quality of PPG compared to ECG chest strap, should be noted [631] as it contributes to higher uncertainty in derived HRV parameters. Early research on using HRV with strength training was promising [129], while the more re- 
cent studies suggest caution for the usefulness of HRV use for assessing the recovery status of the athlete [233, 351, 591, 805].

\section{F. Thermal and circadian measurements}

It is well documented that there is intramuscular temperature dependency on muscle force generation [67, 147, 194], and the core body temperature (CBT) exhibiting a circadian rhythm with an amplitude approximately $1^{\circ} \mathrm{C}$. The CBT is typically highest during the afternoon and lowest in the early morning depending on the intrinsic phase of the individual [34, 439, 542]. Some have even claimed muscle temperature to be probably the most important factor in determining the outcome of exercise performance [234, 649]. However, it is unclear, how much the circadian rhythm per se, generated by 'master circadian oscillator' suprachiasmatic nucleus (SCN) [306], in relation to downstream signals such as temperature, drive the time-of-the-day changes in strength [53, 194] and athletic performance in general [495, 644].

Edwards et al. [206] showed that passive heating and increased humidity was not sufficient to increase strength suggesting some that other intrinsic changes within muscles occur during the day. Some animal studies have suggested that passive heating (e.g. sauna) can stimulate muscle hypertrophy [685], and how it could be used as a mimetic for exercise especially in clinical populations. In "circadian free-running' blind people who are not entrained to the environmental light-dark cycle, it was shown that isometric and isokinetic contraction strength performance mirrored the intrinsic circadian phase of these people, further demonstrating the intrinsic circadian drive in strength production [764]. Zhang et al. [914] showed how maintaining a normal circadian rhythm [341], e.g. regular sleep/wake cycle, can be beneficial for stimulating skeletal muscle repair to prevent or alleviate skeletal muscle atrophy (catabolism).

Thus, it is important to have estimates of the individual circadian phase, core body temperature and muscle temperature during exercise, to better understand the intra- and inter-individual variability to exercise $[644,683]$. One could even argue that all the measures reviewed in this review exhibit a circadian modulation, even if the circadian modulation would not yet had been explicitly studied and reported. Additionally, the menstrual cycle phase in females modulates body temperature variations differently from the circadian drive [440], further complicating the analysis of female athletes.

Rectal temperature recording is considered gold standard in CBT measurement [542], limiting its use for continuous measurement in active athletic population, thus various alternative methods to measure CBT have been proposed. The use of commercial wearable skin surface thermometers called iButtons ${ }^{\circledR}$ worn on multiple locations on body has probably been the most used alternative for rectal measurements [305]. Measurement with iButtons ${ }^{\circledR}$ traditionally required multiple measurement locations, with recent research devoted on reducing this requirement with development of mathematical modeling [208, 427], even going down to single-site measurements from wrist [521], finger [510], and chest with a wearable patch [652].

These continuous and invisible temperature measurement could be used as real-time proxy measurement of changes in circadian phase [167, $190,301,330,772,849$ ], i.e. assessing at what hour the individual athlete's performance peaks allowing individualised training times [196, 277, 727], assessing the efficacy of anti-jetlag interventions when having to compete on a different time zone [669], and facilitating general monitoring of stable circadian entrainment to environmental light/dark cycle [205, 475, 691]. Similar to the challenges in CBT measurement, measuring muscle temperature has been traditionally time-consuming and cumbersome, with novel methods such as insulation disk (INDISK) techniques being proposed to make muscle temperature measurement easier while still physiologically valid [92, 234].

The multisite temperature recording with wearable iButtons ${ }^{\circledR}$ for distal-proximal skin temperature gradient (DPG) measures, and for quantification balance between core and skin temperature [438], can be also approximated optically with non-contact imaging infrared thermography [559]. Imaging infrared thermography have been used in few sports science studies, e.g. for prediction of body part specific injuries [162, 271], as a noninvasive marker for delayed onset muscle soreness (DOMS) [641], and as a measure for activation of motor units in Olympic weightlifting [450].

For sports applications, the recent advances in functional optical fibers [130], could allow smart compressive garments of the future to provide continuous temperature measures for estimation of core body temperature, DPG and intramuscular temperature. Example of such a sensor was developed by Guo et al. [284], who demonstrated a prototype system for real-time measurement of bicep temperature during dumbbell curls. Such a wearable fibre-optic system with non-thermal modalities is demonstrated in Light Lace ${ }^{\circledR}$ smart garment from Organic Robotics (https://www.organicroboticscorp.com/), that integrates measurements of motion capture, muscle activity and respiration into a single garment [894]. 


\section{G. Brain Imaging}

Athlete's focus, fatigue levels, and 'mindmuscle' connection [326, 624] can be monitored in sports application with portable brain imaging techniques such as dry electrode electroencephalography (EEG) [244], or functional near-infrared spectroscopy (fNIRS) [325, 436]. EEG studies have shown how the most fatiguing strength training protocols were associated with greatest increase in cortical activity [232]; how during eccentric contraction, prefrontal cortex seemed to be more involved in the regulation of cortical motor drive compared to isometric and concentric contraction [311]; and how focusing attention on muscle exertion can increase EEG coherence in a cycling task [243]. Recently a magnetic resonance imaging (MRI)-compatible foot pedal device was developed allowing also functional MRI studies for lower extremity movement [192, 281]. Additionally, the mental load quantification from brain imaging might be useful if the weight training with biofeedback is implemented in VR [228, 648], and the mental load could be used to modulate the weight training complexity [263], as done in clinical applications for gait rehabilitation [398].

\section{H. Biochemical biomarkers}

In addition to the electronic measurement techniques outlined above, athletes can be monitored for muscle damage and recovery status [135, 618], using numerous biochemical and multiomics measures [582], sampled from blood, urine or sweat. The sweat-based sampling is the most invisible for the athlete as the developed sensors are typically small [41, 265], around the size of a typical bandage [802]. Lactate and ammonia levels are probably the most interesting for strength training purposes. Lactate levels can be used to track metabolic fatigue and ammonia for assessing neuromuscular fatigue [697]. Additionally, both can be used to track acute recovery between training sets. One of the main remaining challenge in sweat-based sensing has been to ensure the validity between sweat and blood concentrations [255, 668, 910], with recent non-commercial prototypes showing promising results in providing highquality real-time measures of various biomarkers (including lactate and ammonia) [802, 910]. Imani et al. [353] co-fabricated electrochemical lactate sensor and electrophysiological ECG sensor on the same flexible substrate to be mounted on a skin, demonstrating how multimodal wearable sensing systems are feasible even today, and how even more comprehensive sensor solutions could be fabricated in the future.

Hormonal sampling is relatively common in strength training studies, including hormones such as testosterone, human growth hormone (HGH), cortisol, insulin-like growth hormone (IGF-1), cortisol and $17 \beta$-estradiol [437]. Male sex hormone, testosterone [672] is the most frequently used hormone in strength and hypertrophy studies because of its ability to increase muscle mass even in males who do not do resistance training [76]. As a result, its use is considered as doping in professional sports, and therefore banned in competitive sports [886]. Endogenous testosterone exhibit circadian [309], circannual [274] and acute response to heavy training in both men and women [337, 843]. Absolute testosterone levels [681, 682] and acute testosterone response to strength training [484] being lower in females compared to males. The individual circadian profiles (see IIIF above) in testosterone levels [309], can be used to optimise time-of-the-day for strength training for optimal hypertrophy and strength gains.

Female athletes need to consider their menstrual cycle for training programming [622, 682, 687], among other gender differences [318, 682]. Follicular phase typically allows larger strength gains and more hypertrophy [781, 872], while during luteal phase the recovery times from training can be prolonged [515]. The use of hormonal contraceptives does not seem to affect the strength performance in physically active women [572].

The intradaily variation of testosterone levels highlight the need of continuous measurement technology [165], with no wearable solution available at the moment, and, gold standard being based on blood sampling with liquid chromatography-mass spectrometry (LCMS) analysis [110, 157]. Preliminary evidence on electrochemical sensors suggest that in nearfuture, wearable sensing systems could exist for sampling steroid hormones such as cortisol [735, 913], 17 $\beta$-estradiol [793] and testosterone [13].

Creatine kinase $(\mathrm{CK})$ monitoring has been the most commonly used biomarker for assessing muscle damage and recovery, mostly due to its ease of identification and low cost of assays [425]. Total serum CK activity is typically elevated for $24 \mathrm{~h}$ after the exercise, and returns to baseline levels with rest. If the CK levels remain high at rest, a full diagnostic workup should follow for the athlete [93]. The practical problem arises with the definition of "high" CK levels, as total CK levels depend on age, gender, race, muscle mass, hydration level, physical activity, and climatic condition [93]. Additionally, athletes have large inter-individual CK response differences to the same exercise, further complicating the analysis of CK levels for recovery status [93]. Other biomarkers, which can be analysed for exercise recovery include transforming growth factor beta 1 (TGF- $\beta 1$ ) [408], oxylipin [744], brain-derived neurotrophic factor (BNDF), oxygen reduction potential (ORP, redox potential) [647], cell-free DNA (cfDNA) [60], and neutrophil phenotypes [762]. 
In the emerging field of multi-omics [9, 131, 918], relatively little research has done on exercise physiology [187, 564, 582]. Knab et al. (2020) [422] showed how proteomic markers from fingerpricked blood sample could be used to predict the athlete stress and athlete-reported illnesses such as upper respiratory tract infection. Kim et al. (2020) [409] suggested that myostatin A55T genotype was associated with quicker strength recovery following exercise-induced muscle damage. The recently formed Molecular Transducers of Physical Activity in Humans Consortium (MoTrPAC) [700] being one of the most ambitious research programs on the use of omics in exercise physiology. MoTrPAC will attempt to better define omics responses to chronic exercise training at varying exercise intensity levels and exercise modalities.

In general, athlete monitoring would benefit from continuous noninvasive blood sampling [635], as is the case with other measures reviewed here, to better capture the intra-day and long-term variations both within and across athletes. In the future, low-cost continuous noninvasive blood sampling [635] could be done routinely along with automatic gut microbiome analysis [347, 582, 629] with "smart toilet'-type systems [613].

\section{Subjective measures}

In addition to reviewed objective measures, there is tremendous values in asking the athletes themselves to rate the quality of their training sessions, recovery status, and general wellbeing. In the systematic review by Saw et al. [708], it was shown that subjective measures such as mood and perceived stress were shown to outperform blood markers and HR when used as stand-alone measures of sports performance. The authors suggested though that the subjective measures should be combined with more objective measures, and one should not rely solely on subjective measures that might have their own issues with bias and data quality [107, 369].

The rating of perceived effort (RPE) on a scale from 1 to 10 [87, 295, 434, 522], and its strength training specific RPE scale repetitions in reserve (RIR) [48, 824, 933], which estimates how many repetitions you believe you could have done before reaching a technical failure within a set, are probably the most commonly used subjective measures in strength training. These measures are found in advanced gym logger such as Gravitus ${ }^{\circledR}$ (https://gravitus.com/) [314] or Reactive Training Systems' ${ }^{\circledR}$ web app (https://www.reactivetrainingsystems.com/AppHome). Helms et al. [320] for example showed that RPE was an useful tool for prescribing training intensities for powerlifters, in addition to traditional methods such as percentage of 1RM. Larsen et al. [457] also found the subjective markers, especially when combined with objective velocity markers [123], were useful for strength training programming and monitoring. These findings highlight the necessity of subjective measures in precision strength training frameworks for better understanding of the full biopsychosocial profile of the athlete [160, 809].

However, as all subjective measures, RPE scales have their methodological shortcomings [295], and for example Halperin et al. [295] argued that RPE should be accompanied by other subjective measures of affect, fatigue and discomfort, among other measures. The authors proposed the Feeling Scale (FS) , for affective valence in resistancetrained participants with preliminary for real-word use [295] and warranting more detailed followup studies [213]. However, the use of RIR is limited to advanced elites, as its efficient use depends on the ability of athletes to predict maximum effort and being able to separate the perception of effort from actual effort [767], which has been proven to be challenging to intermediate-level lifters [32].

\section{DATASETS FOR STRENGTH TRAINING}

In order to build data-driven precision strength training models, strength training specific datasets need to be acquired first, with relevant modalities and data quality high enough for human movement studies [575, 728]. Currently, no large-scale open-source exercise dataset, or let alone strength training dataset exists (figure 4A), thus we will introduce the concept of self-supervised learning and how to use existing datasets and pre-trained models for custom datasets (figure 4B). Finally, a short overview of technical details relevant for custom dataset acquisition is presented in figure $4 \mathrm{C}$ ).

\section{A. Datasets for exercise and physiotherapy}

To the authors' knowledge, no large-scale opensource databases exist for the development of strength training specific deep learning models. Thus we will review here the existing datasets for generic action recognition models, containing sports activities, physical rehabilitation and the small strength training datasets. Kinematic skeleton databases derived from RGB(-D, D for depth channel) video are the most common type of dataset available [915]. The largest and most frequently used datasets for benchmarking datasets for deep learning model development ${ }^{3}$ are NTU

\footnotetext{
${ }^{3}$ https://paperswithcode.com/task/skeleton-basedaction-recognition
} 


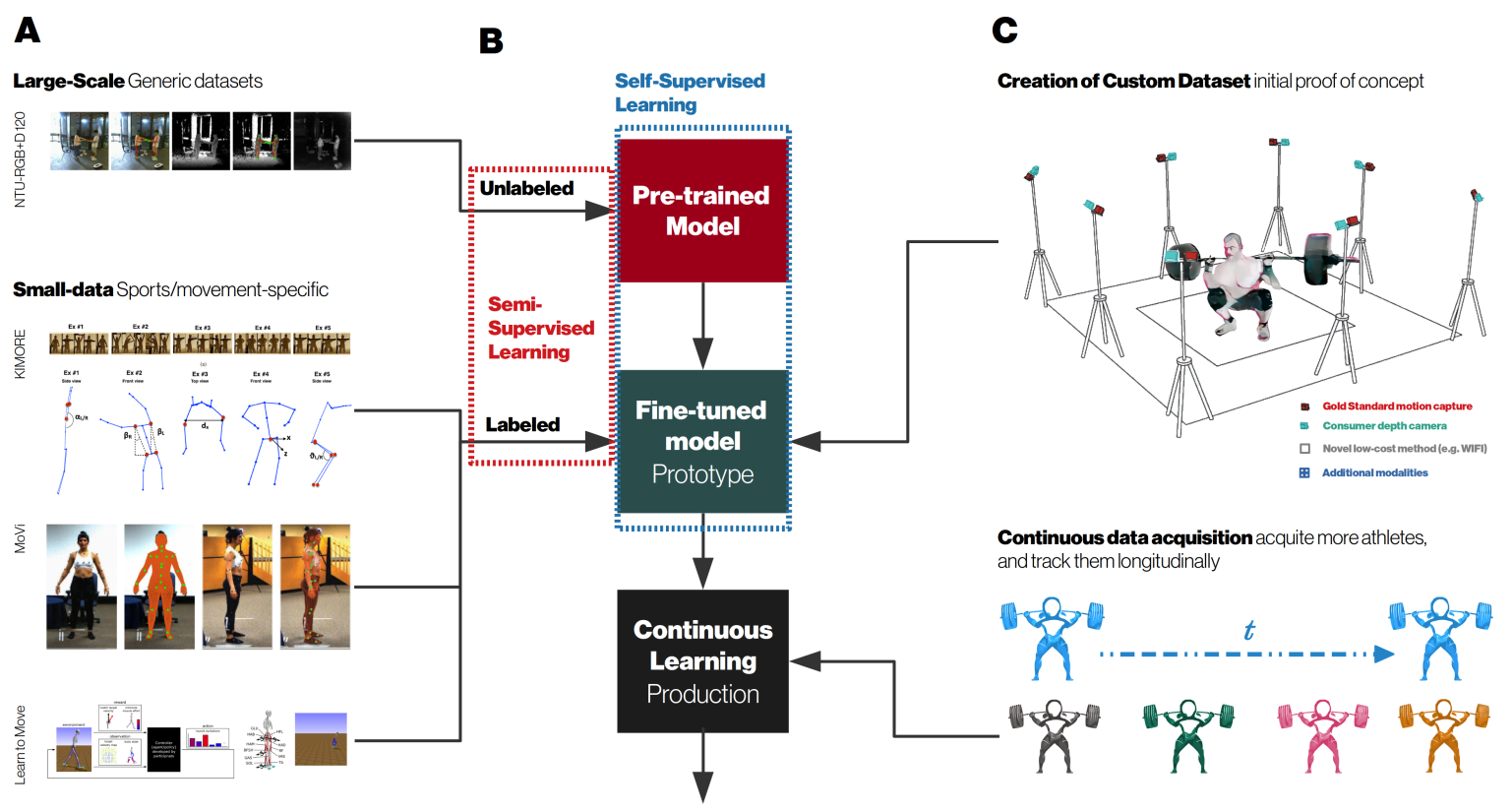

Figure 4. Process of re-purposing available large-scale datasets such as NTU-RGB-D 120 [486] for sports and strength training deep learning development. (A) Large-scale action recognition dataset NTU-RGB-D 120 contains skeleton data of everyday activities which can be used as an initial pre-trained model (B) using recent self-supervised learning approaches that are shown to learn good generic features [932], to be used for finetuning with smaller domain-specific datasets (e.g. KIMORE [111], MoVi [267], Learn to Move [758]) for your desired task. Semi-supervised learning refers to the 'unlabeled' generic datasets that has no sports-specific labels necessarily that are used to supervise the fine-tuning. Physiotherapy datasets such as KIMORE [111] could be used to quickly develop physiotherapy movement assessment models with the self-supervised approach [782], and as no open-source strength training dataset is available, one could capture kinematic data with multiple cameras with different quality levels for example (C). The multiple views reduce the self-occlusion and joint location uncertainty and multiple quality levels allow the simulation of low-quality deployment hardware. After the acquisition of this initial dataset, one wants to continuously acquire new data from more athletes to continuously improve the model and validate the performance of the model.

RGB +D 60 [733, 807], NTU RGB+D 120 [486] and Kinetics-Skeleton [393, 897], all captured with Kinect [14, 148, 626]. The exercise-specific datasets are considerably smaller and are mostly acquired for clinical physiotherapy purposes [477] (see table I), but these datasets could be pooled together as a base dataset for sports specific modeling (figure $4 \mathrm{~A}$ ). It should also be noted that none of the published datasets seem to contain longitudinal recordings for developing athletic training progression models and assessing intra-individual variability.

Capecci et al. [111] published the KIMORE dataset recorded with Kinect ${ }^{\mathrm{TM}}$ consisting of physical rehabilitation body weight exercises recorded with a total of 78 subjects from five different patient groups: 1) healthy, experienced in exercises; 2) healthy, non-experts; 3) stroke rehabilitation; 4) Parkinson's disease; 5) low back pain. Leightley et al. [469] published quite large dataset K3Da containing 54 subjects performing 13 different standardised tests for physiotherapy. AHA-3D dataset [24] contains 21 subjects, both young and elderly subjects, performing body weight exercises. Parisi et al. [606] collected a dataset consisting of 17 volunteers performing high bar back squats, dead- lifts and dumbbell lateral raises, but the dataset was not made public. Cuellar et al. [170] published physical therapy diagnosis exercises with 10 subjects acquired with Kinect. University of Idaho - Physical Rehabilitation Movements Data Set (UI-PRMD) [834] consists of 10 healthy subjects performing rehabilitation exercises such as deep squat, hurdle step and sit to stand captured both with Kinect ${ }^{\mathrm{TM}}$ V2 and Vicon ${ }^{\mathrm{TM}}$ Optical tracking system. Home-based Physical Therapy Exercises (HPTED) consist of only 5 subjects performing shoulder and knee exercises [25]. Militaru et al. [549] collected a dataset small 2,400 image Kaia ${ }^{\mathrm{TM}}$ -style [815] dataset to train a form correction network for plank and holding squat.

For accelerometer-based data, Ebert et al. [203] published a dataset consisting of 8 different body weight exercise with 26 subjects encompassing more than 11,000 exercise repetitions in total, and Taylor et al. [797] published dataset with 3 gym exercises using 9 knee osteoarthritis patients. Reiss and Stricker [665] recorded HR with IMU data for 9 subjects performing physical activities. Kwon et al. [451] addressed the lack of modality-specific training data with their IMUTube system that can convert YouTube videos of human activity into vir- 
Table I. Datasets published for body weight physiotherapy exercises, and gym-based exercises with weights.

\begin{tabular}{|c|c|c|c|c|c|c|c|c|}
\hline \multirow[t]{2}{*}{ Dataset } & \multirow{2}{*}{$\begin{array}{l}\text { Year } \\
2006\end{array}$} & \multirow{2}{*}{$\begin{array}{c}\text { Modalities } \\
\text { EMG }\end{array}$} & \multirow{2}{*}{$\begin{array}{c}\text { Exercise types } \\
\text { Squat }\end{array}$} & \multirow{2}{*}{$\begin{array}{l}n \\
9\end{array}$} & \multicolumn{2}{|c|}{$\begin{array}{r}\text { Exercises Multi- } \\
\text { modal }\end{array}$} & \multicolumn{2}{|c|}{ AvailableRef } \\
\hline & & & & & 3 & - & - & {$[584]$} \\
\hline & 2010 & Accelerometer & Gym exercises & 9 & 3 & - & - & {$[797]$} \\
\hline PAMAP & 2012 & IMU, HR & Everyday activities & 9 & 18 & - & $\checkmark$ & $665]$ \\
\hline HPTED & 2014 & Kinect $^{\circledR}$ & Physiotherapy & 5 & 8 & - & $\checkmark$ & [25] \\
\hline \multirow[t]{2}{*}{ PReSenS } & 2015 & Kinect $^{(B)}$ & Physiotherapy & 10 & 2 & - & - & {$[170]$} \\
\hline & 2015 & Kinect ${ }^{\circledR}$ & $\begin{array}{l}\text { Gym exercises, incl } \\
\text { squat and deadlift }\end{array}$ & 17 & 3 & - & - & {$[606]$} \\
\hline K3Da & 2015 & Kinect ${ }^{\mathbb{B}}$ & Physiotherapy & 54 & 13 & - & $\checkmark$ & [469] \\
\hline \multirow[t]{2}{*}{ EmoPain } & 2016 & $\begin{array}{c}\text { Face videos, } \\
\text { Kinect }^{\mathbb{B}}, \text { EMG }\end{array}$ & Physiotherapy & 50 & 11 & $\checkmark$ & $\checkmark$ & $\begin{array}{l}{[37,} \\
207]\end{array}$ \\
\hline & 2017 & IMU & $\begin{array}{c}\text { Body weight gym } \\
\text { exercises }\end{array}$ & 26 & 8 & - & $\checkmark$ & {$[203]$} \\
\hline MyoGym & 2017 & EMG & Gym exercises & 10 & 30 & - & $\checkmark$ & [431] \\
\hline UI-PRMD & 2018 & Kinect $^{(\mathbb{B}}$, Vicon ${ }^{\text {B }}$ & Physiotherapy & 10 & & - & $\checkmark$ & {$[834$} \\
\hline$A H A-3 D$ & 2018 & Kinect $^{\circledR}$ & Physiotherapy & 21 & 4 & - & $\checkmark$ & {$[24]$} \\
\hline KIMORE & 2019 & Kinect $^{(B)}$ & Physiotherapy & 78 & 5 & - & $\checkmark$ & {$[111]$} \\
\hline \multirow{2}{*}{$\begin{array}{c}\text { PennAction } \\
\text { subset }\end{array}$} & 2019 & RGB video & Squat & $?$ & 1 & - & $\checkmark$ & {$[845]$} \\
\hline & 2020 & Smartphone & Physiotherapy & $?$ & 2 & - & - & [549] \\
\hline MoVi & 2020 & $\begin{array}{l}\text { IMU, Qualisys }^{\circledR}, \\
\text { smartphone, } \\
\text { industrial camera }\end{array}$ & Everyday activities & 90 & 21 & $\checkmark$ & $\checkmark$ & {$[267]$} \\
\hline EV-Action & 2020 & $\begin{array}{c}\text { Kinect }^{\mathbb{B}}, \text { Vicon } \\
\text { EMG }\end{array}$ & Everyday activities & 70 & & $\checkmark$ & $\checkmark$ & {$[856]$} \\
\hline \multirow[t]{2}{*}{ MM-Fit } & 2020 & $\begin{array}{c}\text { RGB-D video, } \\
\text { Smartphone IMU, } \\
\text { smartwatch, earbud }\end{array}$ & Gym exercises & 10 & 10 & $\checkmark$ & $\checkmark$ & {$[774]$} \\
\hline & 2020 & Raptor-E ${ }^{\circledR}$ mocap & Movement screens & 417 & 21 & $\checkmark$ & - & $\begin{array}{l}{[91,} \\
149]\end{array}$ \\
\hline
\end{tabular}

EMG electromyography, IMU inertial measurement unit. Kinect ${ }^{\circledR}$ is an example of low-cost motion capture system, and Vicon ${ }^{\circledR} /$ Qualisys $^{\circledR} /$ Raptor- $E^{\circledR}$ of 'gold standard' level motion capture.

tual streams of IMU data, i.e. synthetic kinematic training data that you could acquire from strength training videos available in abundance.

Ghorbani et al. [267] published an interesting multimodal dataset MoVi that contained both IMU and kinematic data of sports movements from a total of 90 subjects, 60 females and 30 males. Koskimäki et al. [431] published MyoGym dataset containing 10 people doing 30 different gym exercise wearing the $\mathrm{sEMG} \mathrm{Myo}^{\mathrm{TM}}$ armband [431]. Wang et al. [856] combined kinematic data from Vicon $^{\mathrm{TM}}$ and Kinect ${ }^{\mathrm{TM}} \mathrm{V} 2$ with electromyography (EMG) for their EV-Action dataset and recorded 70 subjects performing various actions, not all sports specific. Vyas [845] had used 391 squatting images from PennAction [917] to train an action recognition model for detecting squat exercise. Stömbäck et al. [774] introduced MM-Fit dataset, which was collected using IMUs on smartphones, smartwatches and earbuds worn during gym exercises.

The use of movement screens [441] (e.g. Functional Movement Screen, FMS ${ }^{\mathrm{TM}}$, Functional Movement Systems, USA) has been increasing, especially in sports talent identification in major US sports leagues such as $\mathrm{NBA}^{\mathrm{TM}}$, $\mathrm{NFL}^{\mathrm{TM}}$, $\mathrm{NHL}^{\mathrm{TM}}$ and $\mathrm{MLB}^{\mathrm{TM}}$, often with very subject- ive assessment [530]. Recently, more quantitative and objective athlete approaches have emerged for whole-body movement phenotyping [470, 666, 688]. Clouthier et al. [91, 149] studied a proprietary dataset acquired from 417 athletes, each performing movement tests consisting of 21 unique movements. The authors showed how deep learning seems promising in automating the assessment of movement screens. These types of movement screen datasets, if made public, would be very valuable for sports, physiotherapy and ergonomics assessments.

For musculoskeletal modeling purposes, "Grand Challenge to Predict In Vivo Knee Loads" [239, 412] and "Comprehensive Assessment of the Musculoskeletal System" (CAMS-Knee) [798] provide very specialised datasets for modeling knee contact forces (KCFs) using ground reaction forces, kinematics, EMG, computed tomography (CT), and stationary fluoroscopy data. Imani Nejad et al. [354] studied the difference between OpenSim model predictions and actual measurements from CAMS-Knee, and highlighted the subject-specific variability in musculoskeletal predictions. The 
dataset of "Learn to Move" competition ${ }^{4}$ is not directly on sports movement. However, the task is to develop a deep reinforcement learning controller for 3D human musculoskeletal model, which could be extended to sports and clinical use in the future [758].

\section{B. Finetuning existing datasets for strength training}

In deep learning it is a common practice to take a pre-trained model, initially trained on a largescale generic dataset such as ImageNet [96, 185, $779,908]$, which contain natural images of different dog breeds and flowers. Then, the pre-trained model is repurposed to a custom task with only little data available via fine-tuning (also referred as transfer learning) [38, 137, 570]. Previously the preferred ways for fine-tuning models have been either pre-training the model from scratch using unsupervised learning and then freezing some of the network layers and continuing model training with supervision from your own dataset labels [904], or taking the pre-trained model and freezing all layers but the last layer [264, 512].

Recently, these approaches have been replaced by fine-tuning with self-supervised learning (figure 4B) [38, 134, 278, 370, 424, 791, 921, 932]. In practice self-supervised learning is between unsupervised learning, in the sense that humans do not need to annotate anything, but the learning is supervised with automatically created supervision labels. For example in Bootstrap Your Own Latent (BYOL) approach [278], the supervision targets are synthetically augmented (algoritmically distorted) versions of the input data [65], allowing the network to learn representative features from the large-scale dataset.

Spathis et al. [760] used HR as the (self)supervisory signal for activity data, to learn human activity recognition (HAR), and demonstrated first multimodal self-supervised approach for lifestyle monitoring outperforming unsupervised autoencoders [695]. For pose estimation and future pose prediction, Suris et al. [782] pre-trained first the model on larger Kinetics dataset [393], and then fine-tuned the model on a smaller FineGym dataset [734]. They showed how smaller movement dataset can benefit from an initial self-supervised pre-training first on a larger dataset [932]. This type of training involving both task-specific (labelled) and generic (unlabelled) data, is referred as semi-supervised training [214, 731, 794].

\footnotetext{
${ }^{4}$ NeurIPS 2019: Learn to Move - Walk Around https://github.com/stanfordnmbl/osim-rl
}

\section{Creating your own custom dataset}

With the lack of strength training specific datasets, even the development of proof of concept precision strength training model will require for the sports scientists to acquire a novel custom dataset. Proof-of-concept powerlifting dataset could contain longitudinal trend over a mesocycle (e.g. 4 weeks with 3 weeks of progression, and 1 week of deloading); intra-individual variation during one set, one exercise, one session; and inter-individual variation between athletes for the same exercise prescription in order to capture the information related for the sport in question [876].

The exact technical measurement setup depends on the project resources, but could contain at least kinematic, kinetic, activation and morphological characteristics (III) giving a more complete view of the athlete's training progress (i.e. multimodal model $[145,858])$. The multimodal dataset would also allow the use of classic musculoskeletal modelling techniques [227, 407, 465, 630, 709], and more recent deep reinforcement learning musculoskeletal models [758] for strength training purposes (figure $4 \mathrm{C}$ ). With all the modalities recorded, it is then possible to simulate measurement conditions when not all the modalities are available. This would give an opportunity to demonstrate the relative importance of different modalities [146, 657], how missing data could be generated or handled [289, 290, 331], and how these affects your model's performance. One could additionally include multiple devices of the same modality at various quality levels [384, 448, 759, 783, 920], enabling the quantification of data quality to model performance, e.g. replacing a high-end motion capture system such as Optitrack/Vicon [817] with a Kinect or a smartphone to evaluate how it would affect the model performance $[14,540]$.

Attention should be paid into optimising the quality and ease of use of the data annotation pipeline [150,346], e.g. the spatiotemporal quality of joint locations [575, 728] or temporal segmentation of individual repetitions and during exercises $[75,183,360,688,774,833,857]$. In sports laboratory conditions, the uncertainty of body pose estimation uncertainty could be mitigated by the use of extra infrared retroreflective straps as fiducial markers [128, 156, 230, 405], while the general trend however has been towards markerless motion capture systems [120, 256, 575, 739]. In practice, the use of additional markers is cumbersome for the athlete, and can even increase measurement uncertainty with Kinect ${ }^{\mathrm{TM}}[573,786]$. Colombel et al. [153] recommended the use of miniature markers with a diameter of $2.5 \mathrm{~mm}$ to reduce the interference with Kinect ${ }^{\mathrm{TM}}$ Azure.

It should also be noted that, Kinect ${ }^{\mathrm{TM}} \mathrm{v} 2$ encodes the spine in the skeleton with just 3 joints [870], which might limit the assessment of lumbar- 
pelvic movements (LPM), e.g. the "butt wink" in powerlifting jargon during squat that may load specifically L5-S1 joint [204]. LPM analysis is of a particular interest with low back pain patients [702, 822]. The assessment technology for low back pain, such as recently proposed low-cost electronic skin sensors [919], could be used in strength training and physiotherapy research. [22].

In Kinect ${ }^{\mathrm{TM}}$-based systems it is common to use multiple devices simultaneously to capture the athlete from multiple angles (multiview capture) to reduce self-occlusion and joint location uncertainty $[14,184,372,710,770,816]$. However, in practice the system often would like to be deployed to field with just a single Kinect or a smartphone camera [746, 775]. Depending on the application, the single Kinect ${ }^{\mathrm{TM}}$ might not be provide sufficient joint angle resolution, with Colombel et al. [153] demonstrated the side placement of Kinect ${ }^{\mathrm{TM}}$ Azure devices to giving better signal fidelity over front-facing device. As an example from hand tracking research for VR, Han et al. [300] used 16 separate cameras to obtain the highest hand joint location ground truth. High quality dataset acquisition, with multiple views, modalities and data quality levels, might also help with designing better data augmentation techniques [124, 182, 449, 491, 703], pre-trained networks [853], and defence for adversarial attacks [609], to further improve deep learning performance with small datasets.

\section{Continuous learning}

The researchers should be prepared for the continuous data acquisition, i.e. the iterative improvement of the trained model. In hospitals where the new data is continuously acquired "for free" from patients, but the labelling/annotation of the data by human experts is expensive, active learning systems are frequently used [223, 417, 490]. These systems attempt to predict the most useful (i.e. not a typical patient already found within a labelled dataset) unlabeled data to be labelled by humans for maximising model performance [98, 200, 553]. In strength training, analogous situation could occur when routinely only the kinematic data of every monitored athlete in a given training centre is being recorded, and in the occurrence of any anomalous movement patterns would be tested (compared to existing dataset, i.e. exhibiting high epistemic uncertainty [266, 366]), other modalities such as kinetic, activation, and morphological parameter would also expect to be anomalous and therefore, valuable to be included to the labelled dataset to increase the generalisation capability of the model [201, 506].

When one disseminates the knowledge from the updated model to practice, and from practice back to modeling, these systems are referred to as 'learning health systems' [533, 585]. Similarly one could hope that in the future, learning precision strength training systems would be introduces, in which the developed strength training models would influence strength training practice in the field, and the training practice would influence the work of model development for strength training [865].

Lifelong, or continual learning is a concept often used in autonomous robotics, in which the robot is able to fine-tune its own performance continually through its experience [291, 608, 837]. In physical rehabilitation and in athletic training such a situation could occur with a robotic coach learning the correct movement from physiotherapist via imitation [3, 518], and eventually becoming an autonomous robotic physiotherapist or personal trainer [660], able to help in at-home exercise. Such robotic systems could be pooled together in a federated learning framework, where the training would be decentralised [676, 706, 895, 899, 903], and the individual robots would be considered as "smart edge devices' [552, 800]. Conceptually, this type of distributed system would allow, the heterogeneous coaching style to be pooled into the same model, without the coaches or sports teams/institutes having to give ownership of the data to a model aggregator [823]. The model aggregator, could then be the device manufacturer providing the technology for athlete tracking, or athlete management system (AMS) provider as attempted by Google DeepMind with their Streams EHR for clinical care [638].

The third, similarly sounding concept to the previous ones, is continuous delivery, that refers to software engineering practices to foster automation and, quality and discipline to create workflow for deploying software into production [178, 723, 925]. Often academic studies, are mostly interested in developing new proof-of-concept models, with little thought on real-life software translation and the engineering modules needed for deploying these to "production' [725]. The field focused on improving these deployment practices is referred as $M L O p s$, aiming to unify machine learning (ML) system development (ML + Dev) and ML systems operations (Ops). The concept of MLOps is derived from DevOps that is commonly used software engineering practice making software production and deployment automated and repeatable. What sets MLOps apart from DevOps, is how data and code is managed jointly, as new data can change the behaviour of the system (Software $2.0[553,869])$, whereas in contrast to DevOps in which the program code defines solely the function of the system [251]. 


\section{DEEP LEARNING ENABLED EXERCISE AND STRENGTH TRAINING}

The majority of deep learning studies and startups have targeted recreational gym-goers [145, 850]. For example, algorithms and models have been developed for exercise recognition [401, 587, 646, 651, 747], automatic training diary logging [359, 403, 774], repetition counting [27, 639], adherence monitoring [103], and real-time exercise technique evaluation and correction $[117,133,254$, 349, 371, 372, 589, 607, 610, 845, 900]. The technique assessment models (or motion similarity models $[726,835])$, are the most relevant from these models for our precision strength training assessment for kinematic assessment of the athlete. Notable weight training startups [312] include for example Tonal ${ }^{\mathrm{TM}}$ (https://www.tonal.com/), Tempo $^{\text {TM }}$ (https://tempo.fit/), and Keep ${ }^{\text {TM }}$ (https://www.keepkeep.com/).

Tonal's digital weight system with programmable resistance over the single movement itself [191, 410, 414] is the most interesting of these for precision strength training purposes. One could replace the use of physical chains [583, 846] and elastic bands [19, 202, 381, 447, 516, 662] with digital programming of load changes during one repetition for improving the athlete's lift "sticking points' [429]. Additionally, given an accurate enough individualised neuromuscular model, one could detect fatigue over the training session and dynamically adjust the load for the athlete, e.g., when the training is programmed with RPE [321, 443, 457] (see IIII) or velocity loss targets [861, 862] (see V A 1). This type of a real-time adaptive system could be used as an input for gamified exergames such as StrengthGaming [453], for the clinical rehabilitation and general population to make the training more fun, especially for individuals with adherence issues.

\section{A. Powerlifting: example sports for precision strength training}

Algorithmic approaches specifically for elitelevel S\&C, and competitive strength sports are more scarce than for recreational use (see for example JuggernautAI ${ }^{\mathrm{TM}}$ in section $\S \mathrm{I}$ ). Strength sports involve sports as powerlifting [670], Olympic weightlifting [759], bodybuilding [17], CrossFit ${ }^{\mathrm{TM}}[520]$, strongman competitions [334], arm wrestling and grip sports. Powerlifting seems the most suitable sports from these to illustrate the concept of precision strength training in practice.

In powerlifting [225, 320, 821], the goal is to produce maximum force, in a relatively long time window [829], in the three powerlifting lifts: squat [154, 390], bench press [605, 876] and deadlift
[217, 390]. The effect of lifting technique per se to the amount of load lifted is smaller in powerlifting compared to the effect of lifting technique in Olympic weightlifting [670]. This simplifying the performance monitoring, as the lifted weights reflect more closely the strength performance with less effect from variations in lifting technique. In Olympic Weightlifting, the rate of force development (RFD) is higher than in powerlifting [829, 911], i.e. how explosively the movement is executed, in practice also requiring faster motion cameras than powerlifting. Bodybuilding aims to maximise muscle mass (hypertrophy) with less focus on strength performance [17, 555], and the quantification of muscle mass changes is less straight-forward [308]. Hypertrophy studies would require muscle biopsies and more advanced imaging techniques to properly quantify the progression of the athlete $[1,505]$.

Additionally, powerlifting lift variants are very commonly used by recreational gym-goers and $\mathrm{S} \& \mathrm{C}$ coaches for non-strength sports, making the analysis of powerlifting generalise well to multiple sports. However it should be noted, that not all sports necessarily benefit from powerliftingtype of strength training, i.e. the strength gains do not necessarily transfer to improvements in sports performance [532, 778, 905, 906]. For example, sports requiring explosive power such as boxing [498, 765], taekwondo [485], track and field sprinting [307, 500], and mixed martial arts (MMA) [317, 367, 416, 433, 472, 888], often benefit more from ballistic/plyometric training with a goal to improve athlete's explosive strength capability [829].

Sjöberg et al. [748] conducted an interesting study, with a group of 3 national level Swedish powerlifters, who compiled list of lifting technique issues in squat and deadlift, with relevance for the risk of injury. This list was then evaluated by 14 domain experts consisting of coaches, researchers and competitors within International Powerlifting Federation (IPF), resulting in a new protocol for evaluation of lifting technique. This could be interpreted as the first step of establishing and evidence-based recommendations regarding safe technique in the powerlifting squat and deadlift, and as a qualitative human expert basis to train deep learning networks on movement assessment. The level of inter-rater disagreement and "collective intelligence" [650] between the 14 domain experts furthermore would allow the quantification of uncertainty in lifting assessment, a probabilistic aspect typically desired in deep learning for safety-critical applications such as healthcare [77, 366, 382, 428, 659, 792].

In powerlifting, it is well known qualitatively that body dimensions affect lifting techniques, i.e. how the anthropometric properties of the athlete will affect the definition of 'correct form' [142, 226, 603, 839, 842]. This obviously complic- 
$\mathbf{A}$
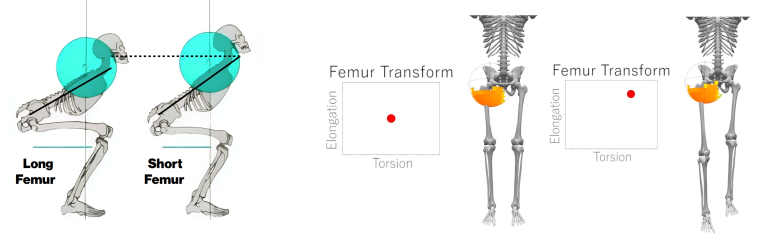

Figure 5. The definition of "gold standard' movement execution is complicated by anthropometric variability in athletes. (A) The athlete with shorter femur compared to trunk, can squat more upright, more easily deeper, and closer to the "textbook standard" of a good squat, whereas the athlete with long femurs has more forward tilting and more cantilever. The athlete with long femurs can try to make the squat deeper for example by placing a block under the heels or using a wider foot stance. In practice, an automatic analysis of squat technique is further complicated by differences in ROM, ankle/hip mobility and tendon/muscle stiffness that we would like to capture for continuously. (B) In computational models such as in the one developed by Ryu et al. [693], it is in theory possible to distinguish these factors, and potentially use it as a framework for athlete monitoring for more fine-grained analysis of technique issues.

ates the development of deep learning models for real-time and offline movement analysis of lifting technique, and increases the data volume requirements (effect size, see e.g. [720, 751] for effect size approximations in deep learning) to capture the inter-individual differences. Anthropometric dependence in Olympic weightlifters was also demonstrated with kinematic data, with the lifters succeeding in competitions with very heterogeneous lifting techniques [171]. In addition to static measures such as trunk/thigh length ratio [246] (5), it might be useful to quantify measures such as ROM [636], tendon stiffness [270], and ankle mobility [246] dynamically in real-time to better understand the dynamic changes during one session and over multiple sessions in movement technique. Additionally, one need to consider the age of the athlete, as deep squat hip kinematics in young athletic adults seem to differ substantially from older and non-athletic subjects [342].

To the knowledge of the authors, no quantitative frameworks have been developed to address the issues of anthropometric variability in lifters for exercise analysis, beyond the suggestion of Cholewa et al. [142] for athletes with greater torso to total length ratios to favour sumostyle deadlifts over conventional style. In practice, the inherent ambiguity in definition of correct form could be modelled for example with deep reinforcement learning (DRL), which have been used medical modeling problems when the exact ground truth is unknown and/or hard to estimate [576, 640, 855]. The developed video-based automatic kinematic assessment could be also used by powerlifting organizations such as IPF to quantify squat depth in real-time, and possibly automating squat depth assessment and reduce subjective judging bias from powerlifiting meets (competitions in powerlifting jargon) [108, 546, 596]. Additionally, the same computer vision techniques could be used in making powerlifting or strongman competitions more spectator-friendly sport with improved sports visualisation [84, 656].

\section{Velocity-based training (VBT)}

Recently, velocity-based training (VBT) and the commercial products related to it have become increasingly popular. VBT is a contemporary method for prescribing strength training based on load/force-velocity curves [481, 557, 861]. One possible application of VBT in training monitoring (see section $\S I I$ ), is to record the velocity of the barbell at different loads (e.g. 45-55-65-75-85\% of 1RM [623]), and use load-velocity curve for the estimation of 1RM [116, 623] (figure 6), without the athletes having to actually lift the 1RM, which would be often counterproductive and increase risk of injury. Mean concentric velocity (MCV) is the most commonly used metric in VBT. As demonstrated by Williams et al. [876] the changes in MCV seem to be more sensitive indicator of neuromuscular fatigue compared to measures of maximal strength production. In other words, the use of VBT could potentially be used to track the internal load of the athlete (see section $§ I I)$, rather than tracking repetitions done at specific loads over the training cycle.

The research interest in VBT has been reflected in the amount of companies offering solutions based on custom camera systems, smartphone cameras, linear transducers or IMUs to measure the velocity of execution. Camera systems are offered by companies such as Perch ${ }^{\mathrm{TM}}$ (https://perch.fit/) and GymAware ${ }^{\text {TM }}$ (https://gymaware.com/) [594, 860]; IMU systems by PUSH ${ }^{\mathrm{TM}}$ band (https://www.trainwithpush.com/) [122, 138]; and smartphone applications by My Lift ${ }^{\mathrm{TM}}$ [47, 519], iLOAD $^{\text {TM }}$ [694], PowerLift ${ }^{\text {TM }}$ [46] and Iron Path TM (http://www.theironpath.com/) [391]; and open-source approaches by Kinovea (https://www.kinovea.org/) [373] and Open Barbell (http://squatsandscience.com/) [272] from RepOne Strength ${ }^{\mathrm{TM}}$ (https://reponestrength.com/). One should pay attention on the possible conflict of interests in the validation studies. For example My Lift ${ }^{\mathrm{TM}}$ app was validated by the app developer himself [47], and external validation study advised against the use of My Lift ${ }^{\mathrm{TM}}$ for VBT [519] due to its excessive velocity errors. In summary, camerabased systems tend to produce more accurate velocity estimates [519,623], and currently they are preferred over IMU systems. 


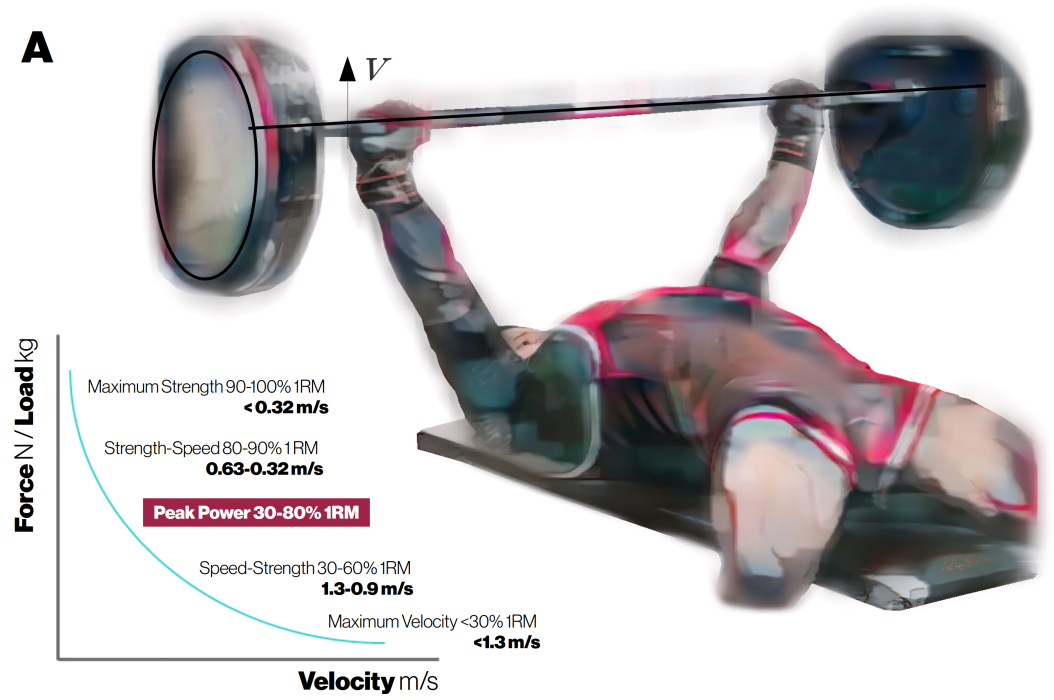

B
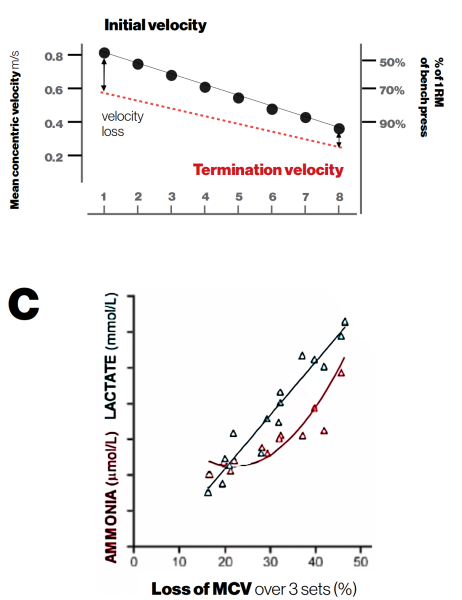

Figure 6. Velocity-based training (VBT) (A) Given a camera or an IMU-based system, one can use the execution velocity (e.g. velocity of the barbell in bench press $[273,698]$ ) to program the training. Bench press velocities across RM range. (B) Example of an 8-week program from Weakley et al. [860] with velocity loss targets for each week, i.e. providing an alternative quantitative target to \% of $1 \mathrm{RM}$, to achieve and not go further on each training session. (C) With real-time sweat-based sensing, one could track metabolic (lactate) and neuromuscular (ammonia) fatigue to estimate optimal inter-set recovery [697]. Figure modified from Wikipedia, licensed under CC BY 2.0 (https://sv.m.wikipedia.org/wiki/Fil:Bench_press_yellow.jpg).

$1 R M 1$-repetition maximum, MCV mean concentric velocity

\section{VISUALISATION, SERIOUS GAMES AND REAL-TIME FEEDBACK}

For both real-time and offline analysis, it is not trivial how the visualisation of an individual training session, and progression over training cycles is implemented. The subtle changes in movement and the associated physiological parameters during one set or the whole training session need to be visualised [69, 121, 487], and made interpretable to human [164, 336], for effective decision making. Conceptually, one could think of extending the video motion magnification techniques from inter-frame difference visualisation [590], to interrepetition/set/session difference visualisations.

For example, Baptista et al. [51] used colour coding for each body segment to visualise the correctness of the movement execution. Adithya et al. [45] rendered the athlete as an 3D avatar [426], and visualised subtle motion as swing trajectories and twisting motion with color coding (See figure $7 \mathrm{~A})$. For general population, and in clinical applications, an interesting outside-the-box visualisation technique is to involve participants in $3 \mathrm{D}$ printing of their activity data (e.g. as food items and allowing better engagement with the exercise or rehabilitation process in a novel and entertaining way as demonstrated by Khot et al. [402] with the Shelfie framework.

Lee et al. $[464]^{5}$ developed a system with the

5 https://youtu.be/a3jfyJ9JVeM
3D avatar being driven by the output of musculoskeletal model (see IIID), i.e. the human motion was driven by the muscle contraction dynamics. The authors demonstrated their model with an avatar doing deadlifts with various loads demonstrating realistic weight-dependent deadlift execution. Additionally, the model allowed the modeling of the effects of pathological muscle weakness and the use of prostheses on biomechanics. In other words, it would be possibly to use the model by Lee et al. [464] with the continuously acquired measures reviewed in section §III as inputs for an athlete-specific musculoskeletal model. The model could be applied in real-time to quantify and visualise sufficient ankle and hip mobility before starting heavy squat exercise and the associated muscle stiffness. As a result, the model could predict suggestions such as an increase in pre-workout warmup or to do a lighter exercise to poor preparedness to train. . Facebook has demonstrated a similar full body tracking model for VR applications with muscle activation simulation along with the kinematic data ${ }^{6}$.

The avatar representation, using common game engines such as Unity [746, 757, 927] or Unreal Engine (UE) [12, 29, 83, 488], transfers also well for home based physical rehabilitation and remote athletic coaching through robotic coach. The robot [35, 689], either a physical robot or an avatar,

\footnotetext{
${ }^{6}$ Facebook 2020 Research: Photorealistic Avatars \&
} Full Body Tracking https://youtu.be/Q-gse_hFkJM( 
A

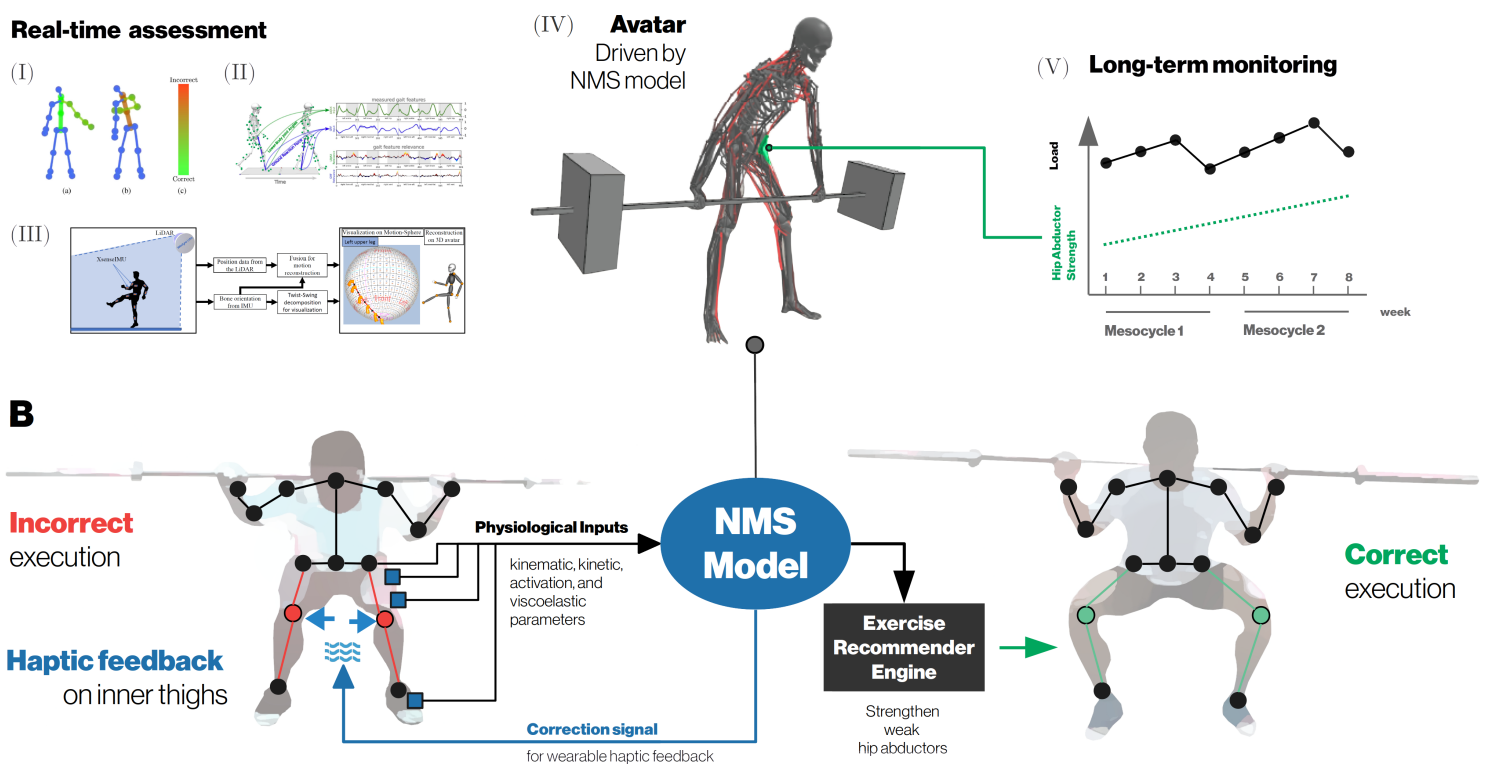

Figure 7. (A) Visualisation for real-time motion (I [51], III [45]) and EMG/GRF (II [6, 339]) assessment, individualised neuromuscular model (NMS) driving 3D avatar. The NMS allows specific muscle groups and its effect to movement to be highlighted (IV [464]). In our simplified example, the athlete has a knee valgus [338], resulting from weak hip abductors, and we can follow longitudinally (V, see e.g. [254, 461]) the efficacy of recommended exercises for strengthening. (B) The NMS model can be used to drive haptic feedback system, implemented for example in a smart compressive garment, and use to provide real-time haptic feedback for the athlete to correct movement execution. In our example case of knee valgus, the athlete might need a targeted strength training program for strengthening the hip abductors (i.e. gluteus medius, gluteus minimus, and tensor fasciae latae) [158] to mitigate knee valgus. The exercise recommender engine (see e.g. [70, 508, 581, 922]), with the NMS in idealised case would be able to detect automatically overall weakness in hip abductors, or sessionspecific issues for example due to poor warm-up or insufficient recovery from previous session possibly leading to increased injury risk, complimentary to human expert assessment [316]. The same model could be then used to visualize the progression in hip abductor strength from acquired data, and display it on a dashboard system for the athlete and the coach [254]. Squat images from John Paul Cauchi's video on mitigating knee valgus https://youtu.be/Lt6OxhEHavQ.

can be trained to learn the physiotherapist's or coach's technique suggestions for that given athlete, and becoming able to autonomously interact with the athlete without human intervention [518]. In addition or as alternative way to improve training annotation, one could record the eye gaze of the supervising coach when observing the movement execution, and input the gaze heatmap as an attention mechanism to the model as a supervision for the robot model training [388, 468]. It could be hypothesised that the gaze of the human expert would be focused on the body parts with technique errors, allowing the deep learning to focus more on those video/joint regions during model training for better performing motion quality assessment model.

\section{A. Serious games}

Recently, physical rehabilitation programs has been increasingly deployed as serious games [86], i.e. the game having utility (seriousness) bey- ond just entertainment [114]. These have been shown the potential to improve patient adherence to rehabilitation $[7,30]$, and have been evaluated through randomised clinical trials (RCTs) [86], and validated for improved clinical outcome measures [524]. Serious games have been well received by the clinicians, especially when co-designed with them [31, 54, 72, 459, 884, 887].

Various exergames, i.e. games with a fitness aspect, have been introduced as games for the general population $[88,222,292,426,453,476,567$, $648,768,859$. The use of gamification and VR have garnered interest also from elite-level sports [10, 198, 420, 578], but in contrast to physiotherapy, the goal in elite-level sports is often not to improve adherence to training, but to provide complimentary training to real life exercises [787], facilitate sports psychology counselling [80], or to provide preventive mental health care $[11,238$, 534, 848]. Ijaz et al. [352] for example showed that VR exergame players can be roughly classified into those that are entertainment-focused (consumers, clinical populations), and those who are exercise- 
focused (e.g. elite athletes), in practice suggesting that the design of games for such too distinct populations might require very different approaches.

In strength training (e.g. in powerlifting training), gamification of heavy squats in virtual reality is not necessarily desired, so for what could VR be used in strength training? Based on practical experience, a fraction of strength athletes do not like to do sufficiently activities supporting recovery from training such as stretching and mobility exercises. Some athletes find these activities boring and have poor adherence to these activities, and could benefit from making them more engaging and fun to do routinely. One could try to gamify such activities with short term goals, for example with visualising the progress in hip and ankle mobility, and the resulting improved squat 1-RM within a causal model. This game could be further designed to include human expert oversight to avoid injuries [871].

\section{B. Real-time feedback methods}

As with visualisation, it is important for the user experience (UX), how the movement quality and the related corrections are communicated in realtime, or offline to the athlete [400] or to the rehabilitation patient [95] after the exercise. The optimal feedback depends on the exercise type, the required mental focus, experience level of the athlete, and the granularity of needed feedback. For example, post-surgery physical rehabilitation patients or beginner yoga practitioners, might be happy with a simple "lower your arms'-type of verbal feedback [602, 661], while others might find it strenuous and needlessly ambiguous [282]. Correctly executed exercise could be rewarded with a simpler auditory feedback such as a click [215], or by a movement sonification such as experimented in physical rehabiliation [579,711], or with music feedback that was shown recently to improve deadlift technique [499]. In sEMG studies, it was shown tha tfocused verbal feedback had a positive impact on activating triceps brachii more than without feedback [249, 604], highlighting the need of some human oversight and encouragement [565, 852].

Haptic feedback is relatively easy to implement integrated to athletic gear for example as smart insoles [118, 212, 368], embedded in smart compressive garment [235, 598] (see figure 7B), in generic clothing [418, 928], or attached around limbs [827, 929]. In gym environments, providing easily digestible visual feedback becomes challenging as the use of large displays cannot be used for better readability, and the UX with a small smartphone or tablet display, as used by the Perch VBT camera system (https://perch.fit/), can be poor during exercise. Visual and haptic feedback in theory allow body part specific feedback in contrast to binary correct vs. incorrect audio click, in which the degree of correctness is hard to communicate also.

Winchester et al. demonstrated how bar path visualisation and verbal feedback was able to improve kinematic and kinetic performance in Olympic weightlifting, both in clean and jerk [879] and in snatch[880]. Elvitigala et al. [212] showed how it is possible to effectively communicate athlete's centre of pressure changes during squat and deadlifts via haptic insoles and visual aids. Turmo Vidal et al. [827] followed a Research through Design approach [259], and evaluated several feedback methods for gym exercises. The authors found athlete-mounted laser light projection to be useful in monitoring planking posture [828], haptic hip feedback useful for monitoring hip tilt in squat, and arm-worn LED light, similar to the earlier 'wearable displays' [260], to be useful for tracking execution speed of barbell curls. They also highlighted the problems of visual feedback for example with squats, where one does not want athletes to make any undesired head movements and gaze changes, that would increase their risk of injury.

The visual feedback has been shown to work well with less intense exercise such as performing Tai Chi exercises at home [806]. Displaying the video of the user next to the instructor with user skeleton overlaid on the user video, was found to be the most effective feedback for skills learning. With the emergence of so-called "smart mirror systems' $[57,74,335,551,611]$, it might be possible to overlay all the relevant feedback on the large-scale mirrors typically found in gym and dance studio environments, that most of the athletes probably will find the most intuitive way of receiving visual feedback as they are accustomed already monitoring their execution from mirrors.

\section{CONCLUSION}

We have outlined the emerging framework of data-driven precision strength training, adopting the data-driven precision from precision medicine, and building on the top of evidence-based personalised strength training to model the intra- and inter-individual variabilities in response to training (see section §II). In practice, despite the recent advances in athlete monitoring technology, long history of data use in sports analytics [531, 536, 550, 714,882 ], and the rise of deep learning, no training system yet exists meeting the expectations of precision medicine as translated to strength training [873]. Some companies are riding the "AI hype train' claiming to have be built AI systems on "world's largest datasets" without any external validation for their claims. This is however not a problem unique for "sports AI' though, with many 
A

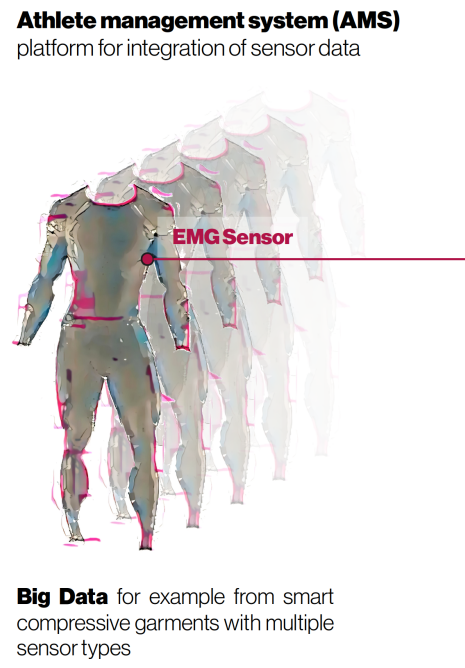

B

Real-time NMS model personalised for the athlete, from the sensors

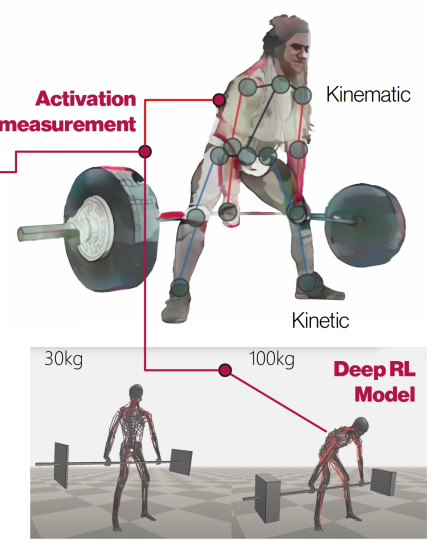

C

\section{Grand Challenges and Benchmark Datasets} Strength training specific datasets needed

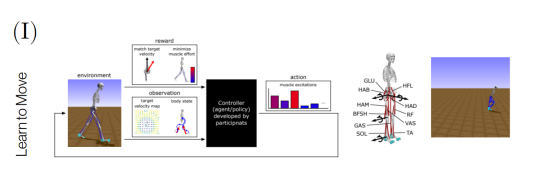

(II)

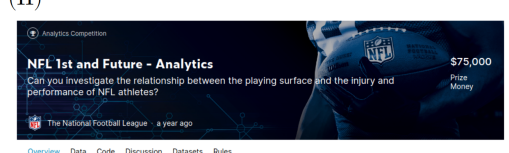

(III)

(IV)

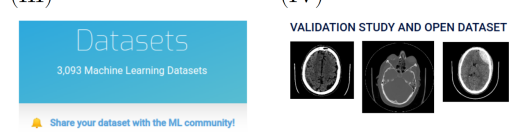

Figure 8. (A) A ubiquitous measurement technology (multimodal sensing) embedded on a smart compressive garment [628]. The new hardware products themselves are not sufficient for precision strength training as exemplified by many failed wearable sensor suits such as Enflux ${ }^{\mathrm{TM}}$ and OMSignal ${ }^{\mathrm{TM}}$ [155]. The hardware require data ingestion pipeline that is easily integrated to larger systems such as athlete management systems (AMS) via APIs. (B) The data from compressive garment would then be used to drive the personalised neuromuscular (NMS) model based on deep reinforcement learning (RL) (see figure 7). That could be used to analyse single training sessions and track the progress of the athlete combining all the measurements of the athlete, e.g. the electrical medical records (EMR) and other self-tracked information. (C) Motivating examples on how to opensource proprietary S\&C datasets for modelling purposes. (I) Learn to Move challenge is targeted for the deep reinforcement learning community, as a shared benchmark to evaluate different modelling ideas, (II) Kaggle competitions (https://www.kaggle.com/) often come with prize money from sponsors, such as in the NFL ${ }^{\mathrm{TM}} 1 \mathrm{st}^{\mathrm{s}}$ and Future to predict injuries (https://www.kaggle.com/c/nfl-playing-surface-analytics), (III) Papers With Code | Machine Learning Datasets provide a centralised database for existing datasets and the published models and their performance on those datasets (https://www.paperswithcode.com/datasets), (IV) Qure.ai (https://qure.ai/) is a radiology startup that open-sourced a small subset of their proprietary data essentially as a marketing for their commercial main product [140].

"disruptive" AI-driven companies taking similar marketing-heavy paths [218, 283, 545, 730].

Main challenge facing the development of precision strength training is the lack of suitable open-source and proprietary datasets (see figure $8 \mathrm{C}$ ). Strength training is waiting for its "fitbitisation", i.e. easy to use measurement device with large-scale adoption, for capturing data at elite, prosumer and recreational levels, at a data quality level pertinent for strength training individualisation. The sheer amount of data tends to be more valuable in medical deep learning, instead of an over-engineered and overfitting model with the fanciest method from the recent trending papers [143, 560, 580, 586]. In practice, the large-scale datasets could be achieved by a massive uptake of smart compressive garments capturing multiple modalities at once (section §III). Examples of simpler smart compressive garments (see figure 8A) are developed by such companies as formsense ${ }^{\mathrm{TM}}$, Athos $^{\mathrm{TM}}$, Myant ${ }^{\mathrm{TM}}$, and asensei ${ }^{\mathrm{TM}}$. Large-scale datasets from similar types of data modalities, such as ergonomics tracking for Amazon ${ }^{\mathrm{TM}}$ warehouse workers to reduce musculoskeletal disorders [777], could be used in self-supervised learning setting to improve the features learned also for strength training modalities.

Additionally, strength training is missing its "grand challenge" / NeurIPS-level benchmark dataset, for which AI and sports science labs would base their models for and compete against each other. Strength training research could draw inspiration from the "Learn to Move' competition, that is aimed at developing new deep reinforcement learning musculoskeletal models for human locomotion [758]. The converge of strength training modeling with musculoskeletal modeling field (see figure 8B) seems likely in near future as the data and model outputs are essentially the same [465, 466, 693].

Often researchers and data-generating institutions are also hesitant in sharing integral part of their intellectual property, and innovative ways to encourage data sharing is needed. One possible avenue for getting some open-source data is to share a subset of the proprietary dataset essentially as a "marketing tool" or as business development ef- 
fort, as done for example by Matterport ${ }^{\mathrm{TM} 7}$ for $3 \mathrm{D}$ indoor data [921], and Qure.ai ${ }^{\mathrm{TM}}$ for intracranial hemorrhage (ICH) CT data [140]. Additionally data-only journals have emerged [176, 719] allowing data-only publications count as published articles in bureaucratic institutional key performance indicators (KPIs), and helping to advance the career of the scientists.

The emerging deep reinforcement learning (DRL) models are most likely being applied first to clinical challenges [673, 707, 758, 890], but with collaborative interest from sports scientists, the models developed e.g. with OpenSIM-RL [404] could be easily retrained for athlete populations. In practice, this could be done through fine-tuning pre-trained robotic and/or clinical locomotion models with self-supervised learning for smaller athletic datasets (IV B). DRL has been studied in medical prescriptive modeling [501, 620], and allows the modeling of intra- and inter-individual responses to exercise (ambiguous ground truths) [855]; and inclusion of the preferences/constraints of the athlete by learning a set of DRL policies [523]. In other words, the model could be constrained by human decisions, such as how the clinical outcome changes if the patient prefers surgery over a prolonged therapy, and how for example athlete's squat could improve given the athlete's preference for rep ranges [458] and exercise selec- tion [90, 94].

In summary, precision strength training is currently lacking large-scale open-source datasets and the measurement technology with good user experience (UX) to facilitate creation of those datasets. While waiting for the pervasive wearable sensor platforms (e.g. smart compressive garments), initial proof-of-concept datasets with laboratory quality technology could be released as 'Grand Challenges', Kaggle competitions or similar benchmarking dataset, to accelerate the strength training research towards the long-term goal of precision strength training. Larger sports training centres and organisations with existing proprietary datasets, who are able to adopt data-centric business strategy, are probably the ones succeeding over the ones simply hiring individual data scientists [56, 126, 547, 658, 732, 741]. Additionally, the most concepts reviewed here are not unique to strength training, and can be adopted in clinical physiotherapy such as in stroke rehabilitation [5], physiotherapy for pain management [430], sports injury rehabilitation [39], osteoarthritis rehabilitation [125], and orthopaedic surgery rehabilitation [79, 241, 474, 799].

\section{FUNDING}

This research did not receive any specific grant from funding agencies in the public, commercial, or not-for-profit sectors.
[1] Abe T, Buckner SL, Mattocks KT, Jessee MB, Dankel SJ, Mouser JG, Bell ZW, Loenneke JP (2018) Skeletal Muscle Mass and Architecture of the World's Strongest Raw Powerlifter: A Case Study. Asian Journal of Sports Medicine 9(2):e61763, https:// dx.doi.org/10.5812/asjsm.61763

[2] Abiad F (2021) Science vs. Bro Science. https:// youtu.be/Hp I3fiK07GA

[3] Abramson J, Ahuja A, Brussee A, Carnevale F, Cassin M, Clark S, Dudzik A, Georgiev P, Guy A, Harley T, Hill F, Hung A, Kenton Z, Landon J, Lillicrap T, Mathewson K, Muldal A, Santoro A, Savinov N, Varma V, Wayne G, Wong N, Yan C, Zhu R (2020) Imitating Interactive Intelligence. arXiv:201205672 [cs] http: //arxiv.org/abs/2012.05672

[4] Abtahi P, Ding V, Yang AC, Bruzzese T, Romanos AB, Murnane EL, Follmer S, Landay JA (2020) Understanding Physical Practices and the Role of Technology in Manual Self-Tracking. Proceedings of the ACM on Interactive, Mobile, Wearable and Ubiquitous Technologies 4(4):115:1-115:24, https: //doi.org/10.1145/3432236

[5] Adans-Dester C, Hankov N, O'Brien A, Vergara-Diaz G, Black-Schaffer R, Zafonte R, Dy J, Lee SI, Bonato $\mathrm{P}$ (2020) Enabling precision rehabilitation interventions using wearable sensors and machine learning to

7 https://matterport.com/blog/announcingmatterport3d-research-dataset track motor recovery. npj Digital Medicine 3(1):1-10, http: //doi.org/10.1038/s41746-020-00328-w

[6] Aeles J, Horst F, Lapuschkin S, Lacourpaille L, Hug F (2020) Revealing the unique features of each individual's muscle activation signatures. bioRxiv p 2020.07.23.217034, https:// doi.org/10.1101/2020.07.23.217034

[7] Afyouni I, Murad A, Einea A (2020) Adaptive Rehabilitation Bots in Serious Games. Sensors 20(24):7037, https://doi.org/10.3390/s20247037

[8] Aguilar M (2021) Apple Watch can help track Parkinson's disease symptoms, study shows. https://ww.statnews.com/2021/02/03/applewatch-parkins ons-disease-tremor /

[9] Ahmed Z (2020) Practicing precision medicine with intelligently integrative clinical and multi-omics data analysis. Human Genomics 14(1):35, https:// doi.org/10.1186/s40246-020-00287-z

[10] Akbaş A, Marszałek W, Kamieniarz A, Polechoński J, Słomka KJ, Juras G (2019) Application of Virtual Reality in Competitive Athletes - A Review. Journal of Human Kinetics 69(1):5-16, http:// doi.org/10.2478/hukin-2019-0023

[11] Åkesdotter C, Kenttä G, Eloranta S, Franck J (2020) The prevalence of mental health problems in elite athletes. Journal of Science and Medicine in Sport 23(4):329-335, http://doi.org/10.1016/ j.jsams.2019.10.022

[12] Alabbasi H, Gradinaru A, Moldoveanu F, Moldoveanu A (2015) Human motion tracking evaluation using Kinect V2 sensor. In: 2015 E-Health and Bioengineering Conference (EHB), pp 1-4, https: 
//doi.org/10.1109/EHB.2015.7391465

[13] Alam MM, Rahman MM, Asiri AM, Fazal MA (2021) A reliable electrochemical approach for detection of testosterone with $\mathrm{CuO}$-doped $\mathrm{CeO} 2$ nanocompositescoated glassy carbon electrode. Journal of Materials Science: Materials in Electronics https://doi.org/ $10.1007 / \mathrm{s} 10854-021-05257-2$

[14] Albert JA, Owolabi V, Gebel A, Brahms CM, Granacher U, Arnrich B (2020) Evaluation of the Pose Tracking Performance of the Azure Kinect and Kinect v2 for Gait Analysis in Comparison with a Gold Standard: A Pilot Study. Sensors 20(18):5104, https://doi.org/10.3390/s20185104

[15] Aliferis C, Adam T (2020) The Landscape of PPM Informatics and the Future of Medicine. In: Adam $\mathrm{T}$, Aliferis C (eds) Personalized and Precision Medicine Informatics: A Workflow-Based View, Health Informatics, Springer International Publishing, Cham, pp 333-343, https://doi.org/10.1007/978-3-03018626-5_21

[16] Álvarez JAE, García JPF, Conceição FAD, JiménezReyes P (2019) Individualized Training Based on Force-Velocity Profiling During Jumping in Ballet Dancers. International Journal of Sports Physiology and Performance 15(6):788-794, https://doi.org/ 10.1123/ijspp.2019-0492

[17] Alves RC, Prestes J, Enes A, de Moraes WMA, Trindade TB, de Salles BF, Aragon AA, SouzaJunior TP (2020) Training Programs Designed for Muscle Hypertrophy in Bodybuilders: A Narrative Review. Sports 8(11):149, https://doi.org/10.3390/ sports 8110149

[18] Andersen TO, Langstrup H, Lomborg S (2020) Experiences With Wearable Activity Data During SelfCare by Chronic Heart Patients: Qualitative Study. Journal of Medical Internet Research 22(7):e15873, http://doi.org/10.2196/15873

[19] Andersen V, Pedersen H, Fimland MS, Shaw MP Solstad TEJ, Stien N, Cumming KT, Saeterbakken AH (2020) Acute Effects of Elastic Bands as Resistance or Assistance on EMG, Kinetics, and Kinematics During Deadlift in Resistance-Trained Men. Frontiers in Sports and Active Living 2, https://dx.doi.org/ 10.3389 /fspor.2020.598284

[20] Andrade R, Wik EH, Rebelo-Marques A, Blanch P, Whiteley R, Espregueira-Mendes J, Gabbett TJ (2020) Is the Acute: Chronic Workload Ratio (ACWR) Associated with Risk of Time-Loss Injury in Professional Team Sports? A Systematic Review of Methodology, Variables and Injury Risk in Practical Situations. Sports Medicine 50(9):1613-1635, https: //doi.org/10.1007/s40279-020-01308-6

[21] Andrade RJ, Freitas SR, Hug F, Le Sant G, Lacourpaille L, Gross R, Quillard JB, McNair PJ, Nordez A (2020) Chronic effects of muscle and nervedirected stretching on tissue mechanics. Journal of Applied Physiology 129(5):1011-1023, https:// doi.org/10.1152/japplphys io1.00239.2019

[22] Androulakis-Korakakis P, Gentil P, Fisher JP, Steele J (2021) Comparison of Isolated Lumbar Extension Strength in Competitive and Noncompetitive Powerlifters, and Recreationally Trained Men. The Journal of Strength \& Conditioning Research 35(3):652-658, http://doi.org/10.1519/JSC.0000000000002722

[23] Angleri V, Oliveira Rd, Biazon T, Damas F, Borghi A, Barroso R, Libardi C (2020) Effects of Drop-Set and Pyramidal Resistance Training Systems on Microvascular Oxygenation: A Near-Infrared Spectroscopy Approach. International Journal of Exercise Science 13(2):1549-1562, https: //digitalcommons.wku.edu/ijes/vol13/iss 2/28

[24] Antunes J, Bernardino A, Smailagic A, Siewiorek D (2018) AHA-3D: A Labelled Dataset for Senior Fit- ness Exercise Recognition and Segmentation from 3D Skeletal Data. In: BMVC

[25] Ar I, Akgul YS (2014) A Computerized Recognition System for the Home-Based Physiotherapy Exercises Using an RGBD Camera. IEEE Transactions on Neural Systems and Rehabilitation Engineering 22(6):1160-1171, https://doi.org/10.1109/ TNSRE.2014.2326254

[26] Aragon AA, Schoenfeld BJ, Wildman R, Kleiner S, VanDusseldorp T, Taylor L, Earnest CP, Arciero PJ, Wilborn C, Kalman DS, Stout JR, Willoughby DS, Campbell B, Arent SM, Bannock L, Smith-Ryan AE, Antonio J (2017) International society of sports nutrition position stand: diets and body composition. Journal of the International Society of Sports Nutrition 14(1):16, https://doi.org/10.1186/s12970$017-0174-y$

[27] Aranguiz D (2015) RecoLift: An Android Wear Fitness Tracker for Strength Training. PhD thesis, University of Illinois at Urbana-Champaign, https:// Ww. ideals.illinois.edu/handle/2142/78982

[28] Araújo D, Couceiro MS, Seifert L, Sarmento H, Davids K (2021) Artificial Intelligence in Sport Performance Analysis. Routledge

[29] Araújo FMAd, Filho PRFV, Filho JAA, Ferreira NMF, Valente A, Soares SFSP (2019) A new approach of developing games for motor rehabilitation using Microsoft Kinect. In: 2019 IEEE 7th International Conference on Serious Games and Applications for Health (SeGAH), pp 1-6, http://doi.org/ 10.1109/SeGAH.2019.8882457

[30] Argent R, Daly A, Caulfield B (2018) Patient Involvement With Home-Based Exercise Programs: Can Connected Health Interventions Influence Adherence? JMIR mHealth and uHealth 6(3):e47, https: //mhealth.jmir.org/2018/3/e47/

[31] Argent R, Slevin P, Bevilacqua A, Neligan M, Daly A, Caulfield B (2019) Wearable Sensor-Based Exercise Biofeedback for Orthopaedic Rehabilitation: A Mixed Methods User Evaluation of a Prototype System. Sensors 19(2):432, https://doi.org/10.3390/ s 19020432

[32] Armes C, Standish-Hunt H, Androulakis-Korakakis P, Michalopoulos N, Georgieva T, Hammond A, Fisher JP, Gentil P, Giessing J, Steele J (2020) "Just One More Rep!" - Ability to Predict Proximity to Task Failure in Resistance Trained Persons. Frontiers in Psychology 11, https://doi.org/10.3389/ fpsyg.2020.565416

[33] Arnold RD, Wade JP (2015) A Definition of Systems Thinking: A Systems Approach. Procedia Computer Science 44:669-678, https://doi.org/10.1016/ j.procs.2015.03.050

[34] Aschoff J (1983) Circadian control of body temperature. Journal of Thermal Biology 8(1):143-147, https: //doi.org/10.1016/0306-4565(83)90094-3

[35] Assad Uz Zaman M, Islam MR, Rahman MH, Schultz K, McGonigle E, Wang I (2020) Robot sensor system for supervised rehabilitation with real-time feedback. Multimedia Tools and Applications 79(35):26643-26660, https://doi.org/10.1007/ s $11042-020-09266-x$

[36] Atkinson G, Batterham AM (2015) True and false interindividual differences in the physiological response to an intervention. Experimental Physiology 100(6):577-588, https://doi.org/10.1113/ep085070

[37] Aung MSH, Kaltwang S, Romera-Paredes B, Martinez B, Singh A, Cella M, Valstar M, Meng H, Kemp A, Shafizadeh M, Elkins AC, Kanakam N, Rothschild Ad, Tyler N, Watson PJ, Williams ACdC, Pantic M, Bianchi-Berthouze N (2016) The Automatic Detection of Chronic Pain-Related Expression: Requirements, Challenges and the Multimodal 
EmoPain Dataset. IEEE Transactions on Affective Computing 7(4):435-451, https://doi.org/10.1109/ TAFFC.2015.2462830

[38] Azizi S, Mustafa B, Ryan F, Beaver Z, Freyberg J, Deaton J, Loh A, Karthikesalingam A, Kornblith S, Chen T, Natarajan V, Norouzi M (2021) Big SelfSupervised Models Advance Medical Image Classification. arXiv:210105224 [cs, eess] http://arxiv.org/ abs / 2101.05224

[39] Ba H (2020) Medical Sports Rehabilitation Deep Learning System of Sports Injury Based on MRI Image Analysis. Journal of Medical Imaging and Health Informatics 10(5):1091-1097, https:// doi.org/10.1166/jmihi.2020.2892

[40] Bailey C (2019) Longitudinal Monitoring of Athletes: Statistical Issues and Best Practices. Journal of Science in Sport and Exercise 1(3):217-227, https: //doi.org/10.1007/s42978-019-00042-4

[41] Baker LB, Model JB, Barnes KA, Anderson ML, Lee SP, Lee KA, Brown SD, Reimel AJ, Roberts TJ, Nuccio RP, Bonsignore JL, Ungaro CT, Carter JM, Li W, Seib MS, Reeder JT, Aranyosi AJ, Rogers JA, Ghaffari R (2020) Skin-interfaced microfluidic system with personalized sweating rate and sweat chloride analytics for sports science applications. Science Advances 6(50):eabe3929, http://doi.org/10.1126/ sciadv.abe3929

[42] Bakker L, Aarts J, Uyl-de Groot C, Redekop W (2020) Economic evaluations of big data analytics for clinical decision-making: a scoping review. Journal of the American Medical Informatics Association 27(9):1466-1475, https://doi.org/10.1093/ jamia/ocaa 102

[43] Bala PC, Eisenreich BR, Yoo SBM, Hayden BY, Park HS, Zimmermann J (2020) Automated markerless pose estimation in freely moving macaques with OpenMonkeyStudio. Nature Communications 11(1):4560, https://doi.org/10.1038/s41467-020$18441-5$

[44] Balagué N, Hristovski R, Almarcha MdC, GarciaRetortillo S, Ivanov PC (2020) Network Physiology of Exercise: Vision and Perspectives. Frontiers in Physiology 11, https://doi.org/10.3389/ fphys.2020.611550

[45] Balasubramanyam A, Patil AK, Chakravarthi B, Ryu JY, Chai YH (2020) Motion-Sphere: Visual Representation of the Subtle Motion of Human Joints. Applied Sciences 10(18):6462, https://doi.org/ 10.3390/app 10186462

[46] Balsalobre-Fernández C, Marchante D, Muñoz-López M, Jiménez SL (2018) Validity and reliability of a novel iPhone app for the measurement of barbell velocity and 1RM on the bench-press exercise. Journal of Sports Sciences 36(1):64-70, https://doi.org/ 10.1080/02640414.2017.1280610

[47] Balsalobre-Fernández C, Geiser G, Krzyszkowski J, Kipp K (2020) Validity and reliability of a computer-vision based smartphone app for measuring barbell trajectory during the snatch. Journal of Sports Sciences 38, http://doi.org/10.1080/ 02640414.2020 .1729453

[48] Balsalobre-Fernández C, Muñoz-López M, Marchante D, García-Ramos A (2021) Repetitions in Reserve and Rate of Perceived Exertion Increase the Prediction Capabilities of the Load-Velocity Relationship. The Journal of Strength \& Conditioning Research 35(3):724-730, http://doi.org/10.1519/ JSC. 0000000000002818

[49] Bansal G, Nushi B, Kamar E, Horvitz E, Weld DS (2020) Optimizing AI for Teamwork. arXiv:200413102 [cs] http://arxiv.org/abs/ 2004.13102

[50] Banyard HG, Nosaka K, Haff GG (2017) Reliabil- ity and Validity of the Load-Velocity Relationship to Predict the 1RM Back Squat. The Journal of Strength \& Conditioning Research 31(7):1897-1904, http://doi.org/10.1519/JSC.0000000000001657

[51] Baptista R, Ghorbel E, Shabayek AER, Moissenet F, Aouada D, Douchet A, André M, Pager J, Bouilland S (2019) Home self-training: Visual feedback for assisting physical activity for stroke survivors. Computer Methods and Programs in Biomedicine 176:111-120, https://doi.org/10.1016/j.cmpb.2019.04.019

[52] Baretta D, Bondaronek P, Direito A, Steca P (2019) Implementation of the goal-setting components in popular physical activity apps: Review and content analysis:. DIGITAL HEALTH https://doi.org/ 10.1177/2055207619862706

[53] Basti A, Yalçin M, Herms D, Hesse J, Aboumanify O, Li Y, Aretz Z, Garmshausen J, El-Athman R, Hastermann M, Blottner D, Relógio A (2021) Diurnal variations in the expression of core-clock genes correlate with resting muscle properties and predict fluctuations in exercise performance across the day. BMJ Open Sport \& Exercise Medicine 7(1):e000876, http://dx.doi.org/10.1136/bmj sem-2020-000876

[54] Baumann AP, O'Neill C, Owens MC, Weber SC, Sivan S, D'Amico R, Carmody S, Bini S, Sawyer AJ, Lotz JC, Goel V, Dmitriev AE (2021) FDA public workshop: Orthopaedic sensing, measuring, and advanced reporting technology (SMART) devices. Journal of Orthopaedic Research 39(1):22-29, https: //doi.org/10.1002/jor.24833

[55] Baz-Valle E, Fontes-Villalba M, Santos-Concejero J (2021) Total Number of Sets as a Training Volume Quantification Method for Muscle Hypertrophy: A Systematic Review. The Journal of Strength \& Conditioning Research 35(3):870-878, http://doi.org/ $10.1519 / \mathrm{JSC} .0000000000002776$

[56] Bean R (2021) Why Is It So Hard to Become a Data-Driven Company? Harvard Business Review https://hbr.org/2021/02/why-is-itso-hard-to-become-a-data-driven-company

[57] Beck M, Crié D (2018) I virtually try it ... I want it ! Virtual Fitting Room: A tool to increase on-line and off-line exploratory behavior, patronage and purchase intentions. Journal of Retailing and Consumer Services 40:279-286, https:// doi.org/10.1016/j.jretconser.2016.08.006

[58] Bekhtaoui W, Sa R, Teixeira B, Singh V, Kirchberg K, Chang Yj, Kapoor A (2020) View Invariant Human Body Detection and Pose Estimation from Multiple Depth Sensors. arXiv:200504258 [cs, eess] http: //arxiv.org/abs/2005.04258

[59] Belbasis A, Fuss FK (2018) Muscle Performance Investigated With a Novel Smart Compression Garment Based on Pressure Sensor Force Myography and Its Validation Against EMG. Frontiers in Physiology 9, https://doi.org/10.3389/fphys.2018.00408

[60] Belcher DJ, Sousa CA, Carzoli JP, Johnson TK, Helms ER, Visavadiya NP, Zoeller RF, Whitehurst M, Zourdos MC (2019) Time course of recovery is similar for the back squat, bench press, and deadlift in well-trained males. Applied Physiology, Nutrition, and Metabolism https://doi.org/10.1139/ apnm-2019-0004

[61] Bell L, Ruddock A, Maden-Wilkinson T, Rogerson D (2020) Overreaching and overtraining in strength sports and resistance training: A scoping review. Journal of Sports Sciences 38(16):1897-1912, https: //doi.org/10.1080/02640414.2020.1763077

[62] Bennie JA, Cocker KD, Smith JJ, Wiesner GH (2020) The epidemiology of muscle-strengthening exercise in Europe: A 28-country comparison including 280,605 adults. PLOS ONE 15(11):e0242220, https: //doi.org/10.1371/journal.pone.0242220 
[63] Bennie JA, Shakespear-Druery J, De Cocker K (2020) Muscle-strengthening Exercise Epidemiology: a New Frontier in Chronic Disease Prevention. Sports Medicine - Open 6(1):40, https://doi.org/10.1186/ s40798-020-00271-w

[64] Bensberg M, Joyce A, Wilson E (2021) Building a Prevention System: Infrastructure to Strengthen Health Promotion Outcomes. International Journal of Environmental Research and Public Health 18(4):1618, https://doi.org/10.3390/ijerph18041618

[65] Benton G, Finzi M, Izmailov P, Wilson AG (2020) Learning Invariances in Neural Networks. arXiv:201011882 [cs, stat] http://arxiv.org/abs/ 2010.11882

[66] Berge AHZ, Kellmann M, Kallweit U, Mir S, Gieselmann A, Meyer T, Ferrauti A, Pfeiffer M, Kölling S (2020) Portable PSG for sleep stage monitoring in sports: Assessment of SOMNOwatch plus EEG. European Journal of Sport Science 20(6):713-721, https://doi.org/10.1080/17461391.2019.1659421

[67] Bergh U, Ekblom B (1979) Influence of muscle temperature on maximal muscle strength and power output in human skeletal muscles. Acta Physiologica Scandinavica 107(1):33-37, https://doi.org/ 10.1111/j.1748-1716.1979.tb06439.x

[68] Berglund M (2020) Hardware is Hard: An exploration of why developers fail to deliver crowdfunded products on time. Master's thesis, Chalmers University of Technology, Gothenburg, Sweden, https: //odr.chalmers.se/handle/20.500.12380/301564

[69] Bernard J, Vögele A, Klein R, Fellner D (2021) Approaches and Challenges in the Visual-interactive Comparison of Human Motion Data. In: Proceedings of the 12th International Joint Conference on Computer Vision, Imaging and Computer Graphics Theory and Applications, pp 217-224, https://doi.org/ $10.5220 / 0006127502170224$

[70] Berndsen J, Smyth B, Lawlor A (2020) Fit to Run: Personalised Recommendations for Marathon Training. In: Fourteenth ACM Conference on Recommender Systems, Association for Computing Machinery, New York, NY, USA, RecSys '20, pp 480485, https://doi.org/10.1145/3383313.3412228

[71] Berner K, Cockcroft J, Morris LD, Louw Q (2020) Concurrent validity and within-session reliability of gait kinematics measured using an inertial motion capture system with repeated calibration. Journal of Bodywork and Movement Therapies 24(4):251-260, https://doi.org/10.1016/j.jbmt.2020.06.008

[72] Bernold G, Matkovic K, Gröller E, Raidou RG (2019) preha: Establishing Precision Rehabilitation with Visual Analytics. In: Kozlíková B, Linsen L, Vázquez PP, Lawonn K, Raidou RG (eds) Eurographics Workshop on Visual Computing for Biology and Medicine, The Eurographics Association, https://doi.org/10.2312/vcbm.20191234

[73] Besomi M, Hodges PW, Clancy EA, Van Dieën J, Hug F, Lowery M, Merletti R, Søgaard K, Wrigley T, Besier T, Carson RG, Disselhorst-Klug C, Enoka RM, Falla D, Farina D, Gandevia S, Holobar A, Kiernan MC, McGill K, Perreault E, Rothwell JC, Tucker K (2020) Consensus for experimental design in electromyography (CEDE) project: Amplitude normalization matrix. Journal of Electromyography and Kinesiology 53:102438, https:// doi.org/10.1016/j.jelekin.2020.102438

[74] Besserer D, Bäurle J, Nikic A, Honold F, Schüssel F, Weber M (2016) Fitmirror: a smart mirror for positive affect in everyday user morning routines. In: Proceedings of the Workshop on Multimodal Analyses enabling Artificial Agents in Human-Machine Interaction, Association for Computing Machinery, New
York, NY, USA, MA3HMI '16, pp 48-55, https: //doi.org/10.1145/3011263.3011265

[75] Bevilacqua A, Brennan L, Argent R, Caulfield B, Kechadi T (2019) Rehabilitation Exercise Segmentation for Autonomous Biofeedback Systems with ConvFSM. In: 41st Annual International Conference of the IEEE Engineering in Medicine and Biology Society (EMBC), IEEE, https://doi.org/10.1109/ EMBC.2019.8856428

[76] Bhasin S, Storer TW, Berman N, Callegari C, Clevenger B, Phillips J, Bunnell TJ, Tricker R, Shirazi A, Casaburi R (1996) The Effects of Supraphysiologic Doses of Testosterone on Muscle Size and Strength in Normal Men. New England Journal of Medicine 335(1):1-7, https://doi.org/10.1056/ NEJM199607043350101

[77] Bhatt U, Antorán J, Zhang Y, Liao QV, Sattigeri P, Fogliato R, Melançon GG, Krishnan R, Stanley J, Tickoo O, Nachman L, Chunara R, Srikumar M, Weller A, Xiang A (2021) Uncertainty as a Form of Transparency: Measuring, Communicating, and Using Uncertainty. arXiv:201107586 [cs] http://arxiv.org/abs/2011.07586

[78] Bica I, Alaa AM, Lambert C, Schaar Mvd (2021) From Real-World Patient Data to Individualized Treatment Effects Using Machine Learning: Current and Future Methods to Address Underlying Challenges. Clinical Pharmacology \& Therapeutics 109(1):87-100, https://doi.org/10.1002/cpt.1907

[79] Bini SA, Schilling PL, Patel SP, Kalore NV, Ast MP, Maratt JD, Schuett DJ, Lawrie CM, Chung CC, Steele GD (2020) Digital Orthopaedics: A Glimpse Into the Future in the Midst of a Pandemic. The Journal of Arthroplasty 35(7):S68-S73, https://doi.org/10.1016/j.arth.2020.04.048

[80] Bird JM (2020) The use of virtual reality headmounted displays within applied sport psychology. Journal of Sport Psychology in Action 11(2):115-128, https://doi.org/10.1080/21520704.2018.1563573

[81] Bjørndal CT, Ronglan LT (2021) Engaging with uncertainty in athlete development - orchestrating talent development through incremental leadership. Sport, Education and Society 26(1):104-116, https: //doi.org/10.1080/13573322.2019.1695198

[82] Blache Y, Michaud B, Rogowski I, Monteil K, Begon M (2019) Sensitivity of Shoulder Musculoskeletal Model Predictions to Muscle-Tendon Properties. IEEE Transactions on Biomedical Engineering 66(5):1309-1317, https://doi.org/10.1109/ TBME.2018.2872833

[83] Black JM, Bradshaw JK, Cokas CA, Ham KH, McNair ED, Rooney BT, Swartz JM (2020) ESPN VR Batting Cage. In: ACM SIGGRAPH 2020 Immersive Pavilion, Association for Computing Machinery, New York, NY, USA, SIGGRAPH '20, pp 1-2, https://doi.org/10.1145/3388536.3407883

[84] Blank P, Groh BH, Eskofier BM (2017) Ball speed and spin estimation in table tennis using a racketmounted inertial sensor. In: Proceedings of the 2017 ACM International Symposium on Wearable Computers, Association for Computing Machinery, New York, NY, USA, ISWC '17, pp 2-9, https: //doi.org/ 10.1145/3123021.3123040

[85] Bonilla DA, Pérez-Idárraga A, Odriozola-Martínez A, Kreider RB (2021) The 4R's Framework of Nutritional Strategies for Post-Exercise Recovery: A Review with Emphasis on New Generation of Carbohydrates. International Journal of Environmental Research and Public Health 18(1):103, https:// doi.org/10.3390/ijerph18010103

[86] Bonnechère B (2018) The Technology. In: Bonnechère B (ed) Serious Games in Physical Rehabilitation: From Theory to Practice, Springer International Pub- 
lishing, Cham, pp 1-9, https://doi.org/10.1007/ 978-3-319-66122-3_1

[87] Borg G (1970) Perceived exertion as an indicator of somatic stress. Scandinavian Journal of Rehabilitation Medicine 2(2):92-98, https:// pubmed.ncbi.nlm.nih.gov/5523831/

[88] Born F, Masuch M, Hahn A (2020) Ghost Sweeper: Using a Heavy Passive Haptic Controller to Enhance a Room-Scale VR Exergame. In: 2020 IEEE Conference on Games (CoG), pp 221-228, https://doi.org/ $10.1109 /$ CoG47356.2020.9231867

[89] Bosse G, Breuer JP, Spies C (2006) The resistance to changing guidelines - what are the challenges and how to meet them. Best Practice \& Research Clinical Anaesthesiology 20(3):379-395, https:// doi.org/10.1016/j.bpa.2006.02.005

[90] Bourne MN, Williams MD, Opar DA, Najjar AA, Kerr GK, Shield AJ (2017) Impact of exercise selection on hamstring muscle activation. British Journal of Sports Medicine 51(13):1021-1028, http: //dx.doi.org/10.1136/bjsports-2015-095739

[91] Boyle A, Ross GB, Graham RB (2020) Machine Learning and Deep Neural Network Architectures for 3D Motion Capture Datasets. In: 2020 42nd Annual International Conference of the IEEE Engineering in Medicine Biology Society (EMBC), pp 4827-4830, https://doi.org/10.1109/EMBC44109.2020.9176426

[92] Brajkovic D, Ducharme MB, Webb P, Reardon FD, Kenny GP (2006) Insulation disks on the skin to estimate muscle temperature. European Journal of Applied Physiology 97(6):761-765, https://doi.org/ $10.1007 / \mathrm{s} 00421-005-0113-5$

[93] Brancaccio P, Maffulli N, Limongelli FM (2007) Creatine kinase monitoring in sport medicine. British Medical Bulletin 81-82:209-230, https://doi.org/ $10.1093 / \mathrm{bmb} / \mathrm{ldm} 014$

[94] Brazil A (2018) A biomechanical framework of the training principles to inform exercise selection within strength and conditioning for sprinting. PhD Thesis, Cardiff Metropolitan University, Cardiff, Wales, https://repository.cardiffmet.ac.uk/handle/ $10369 / 10935$

[95] Brennan L, Dorronzoro Zubiete E, Caulfield B (2020) Feedback Design in Targeted Exercise Digital Biofeedback Systems for Home Rehabilitation: A Scoping Review. Sensors 20(1):181, https: //doi.org/10.3390/s20010181

[96] Brock A, De S, Smith SL, Simonyan K (2021) HighPerformance Large-Scale Image Recognition Without Normalization. arXiv:210206171 [cs, stat] http:// arxiv.org/abs/2102.06171

[97] Buchheit M (2017) Houston, We Still Have a Problem. International Journal of Sports Physiology and Performance 12(8):1111-1114, https://doi.org/ 10.1123/ijspp.2017-0422

[98] Budd S, Robinson EC, Kainz B (2019) A Survey on Active Learning and Human-in-the-Loop Deep Learning for Medical Image Analysis. arXiv:191002923 [cs, eess] http: //arxiv.org/abs/1910.02923

[99] Buford TW, Roberts MD, Church TS (2013) Toward Exercise as Personalized Medicine. Sports Medicine 43(3):157-165, https://doi.org/10.1007/ s40279-013-0018-0

[100] Buongiorno D, Cascarano GD, Brunetti A, De Feudis I, Bevilacqua V (2019) A Survey on Deep Learning in Electromyographic Signal Analysis. In: Huang DS, Huang ZK, Hussain A (eds) Intelligent Computing Methodologies, Springer International Publishing, Cham, Lecture Notes in Computer Science, pp 751-761, https://doi.org/10.1007/978-3-03026766-7_68

[101] Burekhovich SA, Newman JM, Shah NV, Onuoha KO, Le C, Persaud CS, Naziri Q, Sodhi N,
Chaudhri M, Joseph NS, Basu NN, Urban WP, Zikria BA (2018) Epidemiology and Trends of Weightlifting-Related Sprains and Strains that Presented to Emergency Departments in the United States. Journal of Long-Term Effects of Medical Implants 28(2), https://doi.org/10.1615/ JLongTermEf fMed Imp lants . 2018026168

[102] Burle AdQ, Lafayette TBdG, Fonseca JR, Teichrieb V, Gama AEFD (2020) Real-time approach for gait analysis using the Kinect v2 sensor for clinical assessment purpose. In: 2020 22nd Symposium on Virtual and Augmented Reality (SVR), pp 144-153, https://doi.org/10.1109/SVR51698.2020.00034

[103] Burns DM, Leung N, Hardisty M, Whyne CM, Henry $\mathrm{P}$, McLachlin S (2018) Shoulder physiotherapy exercise recognition: machine learning the inertial signals from a smartwatch. Physiological Measurement 39(7):075007, https://doi.org/10.1088/1361-6579/ aacf $d 9$

[104] Busso T, Thomas L (2006) Using Mathematical Modeling in Training Planning. International Journal of Sports Physiology and Performance 1(4):400-405, https://doi.org/10.1123/ijspp.1.4.400

[105] Calhoon G, Fry AC (1999) Injury Rates and Profiles of Elite Competitive Weightlifters. Journal of Athletic Training 34(3):232-238, https: / /ww ncbinlm.nih.gov/pubmed/16558570

[106] Calvert TW, Banister EW, Savage MV, Bach T (1976) A Systems Model of the Effects of Training on Physical Performance. IEEE Transactions on Systems, Man, and Cybernetics SMC-6(2):94-102, https://doi.org/10.1109/TSMC.1976.5409179

[107] Campbell PG, Stewart IB, Sirotic AC, Minett GM (2020) Does exercise intensity affect wellness scores in a dose-like fashion? European Journal of Sport Science 20(10):1395-1404, https: //doi.org/10.1080/ 17461391.2019 .1710264

[108] Candito J (2015) Does The IPF Make Lifters Squat Deeper Than Necessary? https://youtu.be/ zY13tf0ulg

[109] Cao Z, Hidalgo G, Simon T, Wei SE, Sheikh Y (2021) OpenPose: Realtime Multi-Person 2D Pose Estimation Using Part Affinity Fields. IEEE Transactions on Pattern Analysis and Machine Intelligence 43(1):172186, https: //doi.org/10.1109/TPAMI.2019.2929257

[110] Cao ZT, Botelho JC, Rej R, Vesper H (2017) Accuracy-based proficiency testing for testosterone measurements with immunoassays and liquid chromatography-mass spectrometry. Clinica Chimica Acta 469:31-36, https://doi.org/10.1016/ j.cca.2017.03.010

[111] Capecci M, Ceravolo MG, Ferracuti F, Iarlori S, Monteriù A, Romeo L, Verdini F (2019) The KIMORE Dataset: KInematic Assessment of MOvement and Clinical Scores for Remote Monitoring of Physical REhabilitation. IEEE Transactions on Neural Systems and Rehabilitation Engineering 27(7):1436-1448, https://doi.org/10.1109/ TNSRE. 2019.2923060

[112] Capling L, Gifford JA, Beck KL, Flood VM, Halar F, Slater GJ, O'Connor HT (2020) Relative validity and reliability of a novel diet quality assessment tool for athletes: the Athlete Diet Index. British Journal of Nutrition pp 1-13, https://doi.org/ 10.1017/S000711452000416X

[113] Capobianco E (2020) Imprecise Data and Their Impact on Translational Research in Medicine. Frontiers in Medicine 7, https://doi.org/10.3389/ fmed.2020.00082

[114] Caserman P, Hoffmann K, Müller P, Schaub M, Straßburg K, Wiemeyer J, Bruder R, Göbel S (2020) Quality Criteria for Serious Games: Serious Part, Game Part, and Balance. JMIR Serious Games 
8(3):e19037, https://doi.org/10.2196/19037

[115] Cavanagh PR (1974) Electromyography: its use and misuse in physical education. Journal of Health, Physical Education, Recreation 45(5):61-64, https: //doi.org/10.1080/00221473.1974.10614161

[116] Caven EJG, Bryan TJE, Dingley AF, Drury B, Garcia-Ramos A, Perez-Castilla A, Arede J, Fernandes JFT (2020) Group versus Individualised Minimum Velocity Thresholds in the Prediction of Maximal Strength in Trained Female Athletes. International Journal of Environmental Research and Public Health 17(21):7811, https://doi.org/10.3390/ ijerph17217811

[117] Çeliktutan O, Akgul CB, Wolf C, Sankur B (2013) Graph-based analysis of physical exercise actions. In: Proceedings of the 1st ACM international workshop on Multimedia indexing and information retrieval for healthcare, pp 23-32, https://doi.org/10.1145/ 2505323.2505330

[118] Cesini I, Spigler G, Prasanna S, D'Abbraccio J, De Luca D, Dell'Agnello F, Crea S, Vitiello N, Mazzoni A, Oddo CM (2020) Assessment of Intuitiveness and Comfort of Wearable Haptic Feedback Strategies for Assisting Level and Stair Walking. Electronics 9(10):1676, https://doi.org/10.3390/ electronics 9101676

[119] Chalchat E, Gennisson JL, Peñailillo L, Oger M, Malgoyre A, Charlot K, Bourrilhon C, Siracusa J, Garcia-Vicencio S (2020) Changes in the Viscoelastic Properties of the Vastus Lateralis Muscle With Fatigue. Frontiers in Physiology 11, https://doi.org/ 10.3389/fphys. 2020.00307

[120] Challis JH (2021) Motion Analysis. In: Challis $\mathrm{JH}$ (ed) Experimental Methods in Biomechanics, Springer International Publishing, Cham, pp 81-102, https://doi.org/10.1007/978-3-030-52256-8_6

[121] Chan GYY, Nonato LG, Chu A, Raghavan P, Aluru V, Silva CT (2019) Motion Browser: Visualizing and Understanding Complex Upper Limb Movement Under Obstetrical Brachial Plexus Injuries. arXiv:190709146 [csGR] http://arxiv.org/abs/ 1907.09146

[122] Chan YC, Li M, Oymak S (2020) On the Marginal Benefit of Active Learning: Does Self-Supervision Eat Its Cake? arXiv:201108121 [cs] http://arxiv.org/ abs/2011.08121

[123] Chapman M, Larumbe-Zabala E, Triplett NT, Naclerio F (2021) Velocity Change Estimation by Subjective Measures Over a Wide-Load Spectrum in Squat and Bench Press. The Journal of Strength \& Conditioning Research 35:S51, http://doi.org/ 10.1519/JSC. 0000000000003969

[124] Charalambous CC, Bharath AA (2016) A data augmentation methodology for training machine/deep learning gait recognition algorithms. arXiv: 161007570 [cs] http://arxiv.org/abs/1610.07570

[125] Charlton JM, Eng JJ, Li LC, Hunt MA (2021) Learning Gait Modifications for Musculoskeletal Rehabilitation: Applying Motor Learning Principles to Improve Research and Clinical Implementation. Physical Therapy 101(pzaa207), https://doi.org/ 10.1093/ptj/pzaa207

[126] Chase C (2020) The Data Revolution: Cloud Computing, Artificial Intelligence, and Machine Learning in the Future of Sports. In: Schmidt SL (ed) 21st Century Sports: How Technologies Will Change Sports in the Digital Age, Future of Business and Finance, Springer International Publishing, Cham, pp 175-189, https://doi.org/10.1007/978-3-030$50801-210$

[127] Chatfield SC, Volpicelli FM, Adler NM, Kim KL, Jones SA, Francois F, Shah PC, Press RA, Horwitz LI (2019) Bending the cost curve: time series analysis of a value transformation programme at an academic medical centre. BMJ Quality \& Safety 28(6):449-458, http://dx.doi.org/10.1136/bmjqs-2018-009068

[128] Chatzitofis A, Zarpalas D, Kollias S, Daras P (2019) DeepMoCap: Deep Optical Motion Capture Using Multiple Depth Sensors and Retro-Reflectors. Sensors 19(2):282, https://doi.org/10.3390/s19020282

[129] Chen JL, Yeh DP, Lee JP, Chen CY, Huang CY, Lee SD, Chen CC, Kuo TB, Kao CL, Kuo CH (2011) Parasympathetic Nervous Activity Mirrors Recovery Status in Weightlifting Performance After Training. The Journal of Strength \& Conditioning Research 25(6):1546-1552, http://doi.org/10.1519/ JSC.0b013e3181da7858

[130] Chen M, Wang Z, Li K, Wang X, Wei L (2021) Elastic and Stretchable Functional Fibers: A Review of Materials, Fabrication Methods, and Applications. Advanced Fiber Materials https://doi.org/10.1007/ s42765-020-00057-5

[131] Chen R, Snyder M (2013) Promise of personalized omics to precision medicine. WIREs Systems Biology and Medicine 5(1):73-82, https://doi.org/10.1002/ wsbm.1198

[132] Chen RTQ, Rubanova Y, Bettencourt J, Duvenaud D (2019) Neural Ordinary Differential Equations. arXiv:180607366 [cs, stat] http://arxiv.org/ abs/1806.07366

[133] Chen S, Yang RR (2020) Pose Trainer: Correcting Exercise Posture using Pose Estimation. arXiv:200611718 [csCV] https: //arxiv.org/abs/2006.11718v1

[134] Chen T, Kornblith S, Swersky K, Norouzi M, Hinton G (2020) Big Self-Supervised Models are Strong SemiSupervised Learners. arXiv:200610029 [cs, stat] http: //arxiv.org/abs/2006.10029

[135] Cheng AJ, Jude B, Lanner JT (2020) Intramuscular mechanisms of overtraining. Redox Biology 35:101480, https://doi.org/10.1016/ j.redox.2020.101480

[136] Chennaoui M, Arnal PJ, Sauvet F, Léger D (2015) Sleep and exercise: A reciprocal issue? Sleep Medicine Reviews 20:59-72, https://doi.org/10.1016/ j.smrv.2014.06.008

[137] Cheplygina V (2019) Cats or CAT scans: Transfer learning from natural or medical image source data sets? Current Opinion in Biomedical Engineering 9:21-27, https://doi.org/10.1016/j.cobme.2018.12.005

[138] Chéry C, Ruf L (2019) Reliability of the LoadVelocity Relationship and Validity of the PUSH to Measure Velocity in the Deadlift. The Journal of Strength \& Conditioning Research 33(9):2370-2380, http://doi.org/10.1519/JSC.0000000000002663

[139] Chihara T, Sakamoto J (2020) Exerted force estimation using a wearable sensor during manual material handling. Human Factors and Ergonomics in Manufacturing \& Service Industries n/a(n/a), https: //doi.org/10.1002/hfm.20881

[140] Chilamkurthy S, Ghosh R, Tanamala S, Biviji M, Campeau NG, Venugopal VK, Mahajan V, Rao P, Warier P (2018) Deep learning algorithms for detection of critical findings in head CT scans: a retrospective study. The Lancet 392(10162):2388-2396, https://doi.org/10.1016/S0140-6736(18)31645-3

[141] Cho H, Holloway ST, Kosorok MR (2020) Multistage optimal dynamic treatment regimes for survival outcomes with dependent censoring. arXiv:201203294 [statME] https: //arxiv.org/abs/2012.03294v1

[142] Cholewa JM, Atalag O, Zinchenko A, Johnson K, Henselmans M (2019) Anthropometrical Determinants of Deadlift Variant Performance. Journal of Sports Science \& Medicine 18(3):448-453, https: //www.ncbi.nlm.nih.gov/pubmed/31427866 
[143] Christodoulou E, Ma J, Collins GS, Steyerberg EW, Verbakel JY, Calster BV (2019) A systematic review shows no performance benefit of machine learning over logistic regression for clinical prediction models. Journal of Clinical Epidemiology 110:12-22, https: //doi.org/10.1016/j.jclinepi.2019.02.004

[144] Chrzanowski-Smith OJ, Piatrikova E, Betts JA, Williams S, Gonzalez JT (2020) Variability in exercise physiology: Can capturing intra-individual variation help better understand true inter-individual responses? European Journal of Sport Science 20(4):452-460, https://doi.org/10.1080/17461391.2019.1655100

[145] Chu WC, Shih C, Chou W, Ahamed SI, Hsiung $P$ (2019) Artificial Intelligence of Things in Sports Science: Weight Training as an Example. Computer 52(11):52-61, https://doi.org/10.1109/ MC. 2019.2933772

[146] Chung S, Lim J, Noh KJ, Kim G, Jeong H (2019) Sensor Data Acquisition and Multimodal Sensor Fusion for Human Activity Recognition Using Deep Learning. Sensors 19(7):1716, https:// doi.org/10.3390/s 19071716

[147] Clanton TL (2007) Force-calcium relationships in intact skeletal muscle: effects of temperature. The FASEB Journal 21(6):A943-A943, https://doi.org/ 10.1096/fasebj .21.6.A943-a

[148] Clark RA, Mentiplay BF, Hough E, Pua YH (2019) Three-dimensional cameras and skeleton pose tracking for physical function assessment: A review of uses, validity, current developments and Kinect alternatives. Gait \& Posture 68:193-200, https://doi.org/ $10.1016 / j$.gaitpost.2018.11.029

[149] Clouthier AL, Ross GB, Graham RB (2020) Sensor Data Required for Automatic Recognition of Athletic Tasks Using Deep Neural Networks. Frontiers in Bioengineering and Biotechnology 7, https: //doi.org/10.3389/fbioe.2019.00473

[150] Clouthier AL, Ross GB, Mavor MP, Coll I, Boyle A, Graham RB (2021) Development and Validation of a Deep Learning Algorithm and Open-Source Platform for the Automatic Labelling of Motion Capture Markers. IEEE Access pp 1-1, https://doi.org/10.1109/ ACCESS. 2021.3062748

[151] Cobb AD, Roberts SJ, Gal Y (2018) Loss-Calibrated Approximate Inference in Bayesian Neural Networks. arXiv:180503901 [cs, stat] http://arxiv.org/abs/ 1805.03901

[152] Collins FS, Varmus H (2015) A New Initiative on Precision Medicine. New England Journal of Medicine 372(9):793-795, https://doi.org/10.1056/ NEJMp1500523

[153] Colombel J, Daney D, Bonnet V, Charpillet F (2021) Markerless 3D Human Pose Tracking in the Wild with Fusion of Multiple Depth Cameras: Comparative Experimental Study with Kinect 2 and 3. In: Ahad MAR, Inoue S, Roggen D, Fujinami K (eds) Activity and Behavior Computing, Smart Innovation, Systems and Technologies, Springer, Singapore, pp 119-134, https://doi.org/10.1007/978-981-15-8944-7_8

[154] Comfort P, McMahon JJ, Suchomel TJ (2018) Optimizing Squat Technique-Revisited. Strength \& Conditioning Journal 40(6):68-74, http://doi.org/ $10.1519 / \mathrm{SSC} .0000000000000398$

[155] Comstock J (2020) Early smart clothing firm OMSignal is out of business. https: //www.mobihealthnews.com/news/early-smartclothing-firm-omsignal-out-business

[156] Conconi M, Pompili A, Sancisi N, Parenti-Castelli V (2021) Quantification of the errors associated with marker occlusion in stereophotogrammetric systems and implications on gait analysis. Journal of Biomechanics 114:110162, https://doi.org/10.1016/ j.jbiomech.2020.110162

[157] Conklin SE, Knezevic CE (2020) Advancements in the gold standard: Measuring steroid sex hormones by mass spectrometry. Clinical Biochemistry 82:21-32, https://doi.org/10.1016/ j.clinbiochem.2020.03.008

[158] Contreras B, Cordoza G (2019) Glute Lab: The Art and Science of Strength and Physique Training. Victory Belt Publishing, Place of publication not identified

[159] Contreras B, Vigotsky AD (2014) What's All the Fuss About EMG? https://bretcontreras.com/whats fuss-emg/

[160] Cooper JJ, Johnson M, Radcliffe J, Fisher J (2021) Optimal Emotional Profiles for Peak Performance in Strength and Conditioning. The Journal of Strength \& Conditioning Research 35(3):833-840, http:// doi.org/10.1519/JSC.0000000000002832

[161] Correia FD, Nogueira A, Magalhães I, Guimarães J, Moreira M, Barradas I, Teixeira L, Tulha J, Seabra R, Lains J, Bento V (2018) Home-based Rehabilitation With A Novel Digital Biofeedback System versus Conventional In-person Rehabilitation after Total Knee Replacement: a feasibility study. Scientific Reports 8(1):11299, https://doi.org/10.1038/s41598018-29668-0

[162] Côrte AC, Pedrinelli A, Marttos A, Souza IFG, Grava J, Hernandez AJ (2019) Infrared thermography study as a complementary method of screening and prevention of muscle injuries: pilot study. BMJ Open Sport \& Exercise Medicine 5(1):e000431, http:// $\mathrm{dx} . d$ oi.org/10.1136/bmj sem-2018-000431

[163] Couture J (2021) Reflections from the 'Stravasphere': Kudos, community, and (self-)surveillance on a social network for athletes. Qualitative Research in Sport, Exercise and Health 13(1):184-200, https: //doi.org/10.1080/2159676X.2020.1836514

[164] Covert I, Lundberg S, Lee SI (2020) Explaining by Removing: A Unified Framework for Model Explanation. arXiv:201114878 [cs, stat] http://arxiv.org/ abs/2011.14878

[165] Crawford ED, Barqawi AB, O'Donnell C, Morgentaler A (2007) The association of time of day and serum testosterone concentration in a large screening population. BJU International 100(3):509-513, https://doi.org/10.1111/j.1464-410X.2007.07022.x

[166] Creze M, Nordez A, Soubeyrand M, Rocher L, Maître X, Bellin MF (2018) Shear wave sonoelastography of skeletal muscle: basic principles, biomechanical concepts, clinical applications, and future perspectives. Skeletal Radiology 47(4):457-471, https://doi.org/ $10.1007 / \mathrm{s} 00256-017-2843-y$

[167] Crnko S, Schutte H, Doevendans PA, Sluijter JPG, van Laake LW (2020) Minimally Invasive Ways of Determining Circadian Rhythms in Humans. Physiology 36(1):7-20, https://doi.org/10.1152/ physiol.00018.2020

[168] Cruz JA, Wishart DS (2006) Applications of Machine Learning in Cancer Prediction and Prognosis. Cancer Informatics 2:117693510600200030, https:// doi.org/10.1177/117693510600200030

[169] Csabai A (2020) Optimal volume, fatigue accumulation and more ft. Eric Helms \& Mike Israetel (Part II). https://youtu.be/qac2mxPCaoo

[170] Cuellar MP, Ros M, Martin-Bautista MJ, Le Borgne Y, Bontempi G (2015) An Approach for the Evaluation of Human Activities in Physical Therapy Scenarios. In: Agüero R, Zinner T, Goleva R, Timm-Giel A, Tran-Gia P (eds) Mobile Networks and Management, Springer International Publishing, Cham, Lecture Notes of the Institute for Computer Sciences, Social Informatics and Telecommunications Engineering, pp 401-414, https://doi.org/10.1007/978- 
3-319-16292-8_29

[171] Cunanan AJ, Hornsby WG, South MA, Ushakova KP, Mizuguchi S, Sato K, Pierce KC, Stone MH (2020) Survey of Barbell Trajectory and Kinematics of the Snatch Lift from the 2015 World and 2017 Pan-American Weightlifting Championships. Sports 8(9):118, https://doi.org/10.3390/sports 8090118

[172] Czech MD, Psaltos D, Zhang H, Adamusiak T, Calicchio M, Kelekar A, Messere A, Van Dijk KRA, Ramos V, Demanuele C, Cai X, Santamaria M, Patel S, Karahanoglu FI (2020) Age and environmentrelated differences in gait in healthy adults using wearables. npj Digital Medicine 3(1):1-9, https:// doi.org/10.1038/s41746-020-00334-y

[173] Dajime PF, Smith H, Zhang Y (2020) Automated classification of movement quality using the Microsoft Kinect V2 sensor. Computers in Biology and Medicine 125:104021, https://doi.org/10.1016/ j.compbiomed.2020.104021

[174] Dankel SJ, Loenneke JP (2020) A Method to Stop Analyzing Random Error and Start Analyzing Differential Responders to Exercise. Sports Medicine 50(2):231-238, https://doi.org/10.1007/ s40279-019-01147-0

[175] Danter S (2020) Command and Control: The Quantified Self and Biomedical Transhumanism. In: Reichardt U, Schober R (eds) Laboring Bodies and the Quantified Self, transcript, pp 15-40, https: //doi.org/10.14361/9783839449219-002

[176] Data S (2019) Five years of Scientific Data. Scientific Data 6(1):72, https://doi.org/10.1038/ s41597-019-0065-y

[177] Daunhawer I, Sutter TM, Marcinkevi?cs R, Vogt JE (2020) Self-supervised Disentanglement of Modalityspecific and Shared Factors Improves Multimodal Generative Models. In: GCPR 2020, p 15

[178] Davenport T, Malone K (2021) Deployment as a Critical Business Data Science Discipline. Harvard Data Science Review https://doi.org/10.1162/ $99608 f 92.90814$ c32

[179] Davies A, Allman-Farinelli M, Owen K, Signal L, Hosking C, Wang L, Bauman A (2020) Feasibility Study Comparing Physical Activity Classifications from Accelerometers with Wearable Camera Data. International Journal of Environmental Research and Public Health 17(24):9323, https:// doi.org/10.3390/ijerph17249323

[180] Davies D (2021) How the Royal Ballet is Using Sports Science to Make Dancers More Durable, Stronger and Fitter. Men's Health https://www.menshealth.com/uk/fitness/ a35223268/sports-science-at-the-royal-ballet/

[181] Davis JD, Kumbale CM, Zhang Q, Voit EO (2019) Dynamical systems approaches to personalized medicine. Current Opinion in Biotechnology 58:168-174, https://www.sciencedirect.com/ science/article/pii/S0958166918301915

[182] Dawar N, Ostadabbas S, Kehtarnavaz N (2019) Data Augmentation in Deep Learning-Based Fusion of Depth and Inertial Sensing for Action Recognition. IEEE Sensors Letters 3(1):1-4, https://doi.org/ 10.1109/LSENS.2018.2878572

[183] Deng D, Wu J, Wang J, Wu Y, Xie X, Zhou Z, Zhang H, Zhang X, Wu Y (2021) EventAnchor: Reducing Human Interactions in Event Annotation of Racket Sports Videos. arXiv:210104954 [cs] http: //arxiv.org/abs/2101.04954

[184] Deng H, Bui M, Navab N, Guibas L, Ilic S, Birdal T (2020) Deep Bingham Networks: Dealing with Uncertainty and Ambiguity in Pose Estimation. arXiv:201211002 [cs] https://arxiv.org/abs/ 2012.11002v1

[185] Deng J, Dong W, Socher R, Li L, Kai Li, Li Fei-
Fei (2009) ImageNet: A large-scale hierarchical image database. In: 2009 IEEE Conference on Computer Vision and Pattern Recognition, pp 248-255, https: //doi.org/10.1109/CVPR.2009.5206848

[186] Derungs A, Amft O (2020) Estimating wearable motion sensor performance from personal biomechanical models and sensor data synthesis. Scientific Reports 10(1):11450, https://doi.org/10.1038/s41598-020 68225-6

[187] Deshmukh AS, Steenberg DE, Hostrup M, Birk JB, Larsen JK, Santos A, Kjøbsted R, Hingst JR, Schéele CC, Murgia M, Kiens B, Richter EA, Mann M, Wojtaszewski JFP (2021) Deep muscle-proteomic analysis of freeze-dried human muscle biopsies reveals fiber type-specific adaptations to exercise training. Nature Communications 12(1):304, http://doi.org/ $10.1038 / \mathrm{s} 41467-020-20556-8$

[188] Di Giminiani R, Lancia S, Ferrari M, Quaresima V, Tilma Vistisen H, Kliltgaard A, Arbjerg Heick R, Oestergard K, Yndgaard Soerensen K, Cardinale M (2018) A wearable integrated textile EMG and muscle oximetry system for monitoring exercise-induced effects: a feasibility study. In: 2018 IEEE International Symposium on Medical Measurements and Applications (MeMeA), pp 1-5, https://doi.org/10.1109/ MeMeA.2018.8438785

[189] Di Giminiani R, Cardinale M, Ferrari M, Quaresima V (2020) Validation of Fabric-Based Thigh-Wearable EMG Sensors and Oximetry for Monitoring Quadriceps Activity during Strength and Endurance Exercises. Sensors 20(17):4664, https://doi.org/10.3390/ s20174664

[190] Dijk DJ, Duffy JF (2020) Novel Approaches for Assessing Circadian Rhythmicity in Humans: A Review. Journal of Biological Rhythms 35(5):421-438, https://doi.org/10.1177/0748730420940483

[191] Dong S, Lu KQ, Sun JQ, Rudolph K (2006) Adaptive Force Regulation of Muscle Strengthening Rehabilitation Device With Magnetorheological Fluids. IEEE transactions on neural systems and rehabilitation engineering : a publication of the IEEE Engineering in Medicine and Biology Society 14(1):55-63, https://dx.doi.org/10.1109/TNSRE.2005.863839

[192] Doolittle JD, Downey RJ, Imperatore JP, Dowdle LT, Lench DH, McLeod J, McCalley DM, Gregory CM, Hanlon CA (2021) Evaluating a novel MRcompatible foot pedal device for unipedal and bipedal motion: Test-retest reliability of evoked brain activity. Human Brain Mapping 42(1):128-138, https: //doi.org/10.1002/hbm.25209

[193] Dorsey A, Scherer E, Eckhoff R, Furberg RD (2020) Measurement of Human Stress: A Multidimensional Approach. PsyArXiv https://psyarxiv.com/es2p9/

[194] Douglas CM, Hesketh SJ, Esser KA (2020) Time of Day and Muscle Strength: A Circadian Output? Physiology 36(1):44-51, https://doi.org/10.1152/ physiol.00030.2020

[195] Drăgulinescu A, Drăgulinescu AM, Zincă G, Bucur D, Feie? V, Neagu DM (2020) Smart Socks and In-Shoe Systems: State-of-the-Art for Two Popular Technologies for Foot Motion Analysis, Sports, and Medical Applications. Sensors 20(15):4316, https: //doi.org/10.3390/s20154316

[196] Drust B, Waterhouse J, Atkinson G, Edwards B, Reilly $\mathrm{T}$ (2005) Circadian Rhythms in Sports Performance-an Update. Chronobiology International 22(1):21-44, https://doi.org/10.1081/CBI200041039

[197] Duchateau J, Stragier S, Baudry S, Carpentier A (2021) Strength Training: In Search of Optimal Strategies to Maximize Neuromuscular Performance. Exercise and Sport Sciences Reviews 49(1):2-14, http://doi.org/10.1249/JES.0000000000000234 
[198] Düking P, Holmberg HC, Sperlich B (2018) The Potential Usefulness of Virtual Reality Systems for Athletes: A Short SWOT Analysis. Frontiers in Physiology 9, https://doi.org/10.3389/ fphys.2018.00128

[199] Dunning J, Butts R, Henry N, Mourad F, Brannon A Rodriguez H, Young I, Arias-Buría JL, Fernández-delas Peñas C (2018) Electrical dry needling as an adjunct to exercise, manual therapy and ultrasound for plantar fasciitis: A multi-center randomized clinical trial. PLOS ONE 13(10):e0205405, https://doi.org/ 10.1371/journal.pone.0205405

[200] Dupre R, Fajtl J, Argyriou V, Remagnino P (2020) Improving Dataset Volumes and Model Accuracy With Semi-Supervised Iterative Self-Learning. IEEE Transactions on Image Processing 29:4337-4348, https://doi.org/10.1109/TIP.2019.2913986

[201] Dziugaite GK, Drouin A, Neal B, Rajkumar N, Caballero E, Wang L, Mitliagkas I, Roy DM (2020) In Search of Robust Measures of Generalization. arXiv:201011924 [cs, stat] http://arxiv.org/abs/ 2010.11924

[202] Ebben WP, Jensen RL (2002) Electromyographic and kinetic analysis of traditional, chain, and elastic band squats. Journal of Strength and Conditioning Research 16(4):547-550

[203] Ebert A, Marouane C, Ungnadner C, Klein A (2017) An Open, Labeled Dataset for Analysis and Assessment of Human Motion. In: Wireless Mobile Communication and Healthcare, Springer, Cham, pp 99-106, https://doi.org/10.1007/978-3-319-98551-0_12

[204] Edington C (2017) Lumbar spine kinematics and kinetics during heavy barbell squat and deadlift variations. PhD Thesis, University of Saskatchewan Saskatoon, http://hdl.handle.net/10388/8539

[205] Edwards BJ, Reilly T, Waterhouse J (2009) Zeitgeber-effects of exercise on human circadian rhythms: what are alternative approaches to investigating the existence of a phase-response curve to exercise? Biological Rhythm Research 40(1):53-69, https://doi.org/10.1080/09291010802067072

[206] Edwards BJ, Pullinger SA, Kerry JW, Robinson WR, Reilly TP, Robertson CM, Waterhouse JM (2013) Does Raising Morning Rectal Temperature to Evening Levels Offset the Diurnal Variation in Muscle Force Production? Chronobiology International 30(4):486-501, https://doi.org/10.3109/ 07420528.2012 .741174

[207] Egede JO, Song S, Olugbade TA, Wang C, Williams A, Meng H, Aung M, Lane ND, Valstar M, Bianchi-Berthouze N (2020) EMOPAIN Challenge 2020: Multimodal Pain Evaluation from Facial and Bodily Expressions. arXiv:200107739 [cs, eess] http: //arxiv.org/abs/2001.07739

[208] Eggenberger P, MacRae BA, Kemp S, Bürgisser M, Rossi RM, Annaheim S (2018) Prediction of Core Body Temperature Based on Skin Temperature, Heat Flux, and Heart Rate Under Different Exercise and Clothing Conditions in the Heat in Young Adult Males. Frontiers in Physiology 9, https://doi.org/ 10.3389/f phys. 2018.01780

[209] Elkholy A, Hussein ME, Gomaa W, Damen D, Saba E (2020) Efficient and Robust Skeleton-Based Quality Assessment and Abnormality Detection in $\mathrm{Hu}-$ man Action Performance. IEEE Journal of Biomedical and Health Informatics 24(1):280-291, https: //doi.org/10.1109/JBHI.2019.2904321

[210] Elliott M, Ballantyne J, Rouse T, Leidersdorf E (2015) Generation of personalized training regimens from motion capture data. https:// patents.google.com/patent/US9161708B2/en

[211] Elmore JG, Barton MB, Moceri VM, Polk S, Arena PJ, Fletcher SW (1998) Ten-Year Risk of
False Positive Screening Mammograms and Clinical Breast Examinations. New England Journal of Medicine 338(16):1089-1096, https://doi.org/10.1056/ NEJM199804163381601

[212] Elvitigala DS, Matthies DJ, David L, Weerasinghe C, Nanayakkara S (2019) GymSoles: Improving Squats and Dead-Lifts by Visualizing the User's Center of Pressure. In: Proceedings of the 2019 CHI Conference on Human Factors in Computing Systems, Association for Computing Machinery, New York, NY, USA, CHI '19, pp 1-12, https://doi.org/10.1145/ 3290605.3300404

[213] Emanuel A, Smukas IR, Halperin I (2020) How One Feels During Resistance Exercises: A Repetitionby-Repetition Analysis Across Exercises and Loads. International Journal of Sports Physiology and Performance -1(aop):1-10, http://doi.org/10.1123/ ijspp. 2019-0733

[214] van Engelen JE, Hoos HH (2020) A survey on semisupervised learning. Machine Learning 109(2):373440, https://doi.org/10.1007/s10994-019-05855-6

[215] Ennett TM, Zonneveld KLM, Thomson KM, Vause T, Ditor D (2020) Comparison of two TAGteach error-correction procedures to teach beginner yoga poses to adults. Journal of Applied Behavior Analysis 53(1):222-236, https://doi.org/10.1002/jaba.550

[216] Enoka RM, Duchateau J (2015) Inappropriate interpretation of surface EMG signals and muscle fiber characteristics impedes understanding of the control of neuromuscular function. Journal of Applied Physiology 119(12):1516-1518, https:// doi.org/10.1152/japplphysio1.00280.2015

[217] Escamilla R, Lowry T, Osbahr D, Speer K (2001) Biomechanical analysis of the deadlift during the 1999 special olympics world games. ISBS - Conference Proceedings Archive https://ojs.ub.uni-konstanz.de/ cpa/article/view/3819

[218] Esmaeilzadeh P (2020) Use of AI-based tools for healthcare purposes: a survey study from consumers' perspectives. BMC Medical Informatics and Decision Making 20(1):170, https://doi.org/10.1186/ s 12911-020-01191-1

[219] Esmonde K (2020) 'There's only so much data you can handle in your life': accommodating and resisting self-surveillance in women's running and fitness tracking practices. Qualitative Research in Sport, Exercise and Health 12(1):76-90, https://doi.org/10.1080/ 2159676X.2019.1617188

[220] Faltaous S, Neuwirth J, Gruenefeld U, Schneegass S (2020) SaVR: Increasing Safety in Virtual Reality Environments via Electrical Muscle Stimulation. In: 19th International Conference on Mobile and Ubiquitous Multimedia, Association for Computing Machinery, New York, NY, USA, pp 254-258, https: //doi.org/10.1145/3428361.3428389

[221] Falzer PR (2020) Evidence-based medicine's curious path: From clinical epidemiology to patient-centered care through decision analysis. Journal of Evaluation in Clinical Practice n/a(n/a), https://doi.org/ $10.1111 / \mathrm{jep} .13466$

[222] Farič N, Potts HWW, Hon A, Smith L, Newby K, Steptoe A, Fisher A (2019) What Players of Virtual Reality Exercise Games Want: Thematic Analysis of Web-Based Reviews. Journal of Medical Internet Research 21(9):e13833, https://doi.org/10.2196/13833

[223] Farquhar S, Gal Y, Rainforth T (2021) On Statistical Bias In Active Learning: How and When To Fix It. arXiv:210111665 [cs, stat] http://arxiv.org/abs/ 2101.11665

[224] Feigin M, Zwecker M, Freedman D, Anthony BW (2020) Detecting muscle activation using ultrasound speed of sound inversion with deep learning. In: 2020 42nd Annual International Conference of 
the IEEE Engineering in Medicine Biology Society (EMBC), pp 2092-2095, https://doi.org/10.1109/ EMBC44109.2020.9175237

[225] Ferland PM, Comtois AS (2019) Classic Powerlifting Performance: A Systematic Review. Journal of Strength and Conditioning Research 33 Suppl 1:S194-S201, https://doi.org/10.1519/jsc.0000000000003099

[226] Ferland PM, Laurier A, Comtois AS (2020) Relationships Between Anthropometry and Maximal Strength in Male Classic Powerlifters. International Journal of Exercise Science 13(4):1512-1531, https: //digitalcommons.wku.edu/ijes/vol13/iss4/32

[227] Fernandez J, Dickinson A, Hunter P (2020) Population based approaches to computational musculoskeletal modelling. Biomechanics and Modeling in Mechanobiology 19(4):1165-1168, https://doi.org/ $10.1007 / s 10237-020-01364-x$

[228] Fernandez JA, Fusté A, Richer R, Maes P (2019) Deep reality: an underwater VR experience to promote relaxation by unconscious HR, EDA, and brain activity biofeedback. In: ACM SIGGRAPH 2019 Virtual, Augmented, and Mixed Reality, Association for Computing Machinery, New York, NY, USA, SIGGRAPH '19, p 1, https://doi.org/10.1145/ 3306449.3328818

[229] Filipovic A, Kleinöder H, Dörmann U, Mester J (2012) Electromyostimulation-A Systematic Review of the Effects of Different Electromyostimulation Methods on Selected Strength Parameters in Trained and Elite Athletes. The Journal of Strength \& Conditioning Research 26(9):2600-2614, http://doi.org/ 10.1519/JSC.0b013e31823f2cd1

[230] Fiorentino NM, Atkins PR, Kutschke MJ, Bo Foreman K, Anderson AE (2020) Soft tissue artifact causes underestimation of hip joint kinematics and kinetics in a rigid-body musculoskeletal model. Journal of Biomechanics 108:109890, https: //doi.org/10.1016/j.jbiomech.2020.109890

[231] Fister I, Ljubič K, Suganthan PN, Perc M, Fister I (2015) Computational intelligence in sports: Challenges and opportunities within a new research domain. Applied Mathematics and Computation 262:178-186, https://doi.org/10.1016/ j.amc. 2015.04.004

[232] Flanagan SD, Dunn-Lewis C, Comstock BA, Maresh CM, Volek JS, Denegar CR, Kraemer WJ (2012) Cortical Activity during a Highly-Trained Resistance Exercise Movement Emphasizing Force, Power or Volume. Brain Sciences 2(4):649-666, https:// doi.org/10.3390/brainsci2040649

[233] Flatt AA, Globensky L, Bass E, Sapp BL, Riemann BL (2019) Heart Rate Variability, Neuromuscular and Perceptual Recovery Following Resistance Training. Sports 7(10):225, https://doi.org/10.3390/ sports 7100225

[234] Flouris AD, Dinas PC, Tsitoglou K, Patramani I, Koutedakis Y, Kenny GP (2015) Non-invasive measurement of tibialis anterior muscle temperature during rest, cycling exercise and post-exercise recovery. Physiological Measurement 36(7):N103-N113, https : //doi.org/10.1088/0967-3334/36/7/n103

[235] Foo EW, Lee JW, Compton C, Ozbek S, Holschuh B (2019) User experiences of garment-based dynamic compression for novel haptic applications. In: Proceedings of the 23rd International Symposium on Wearable Computers, Association for Computing Machinery, New York, NY, USA, ISWC '19, pp 5459, https://doi.org/10.1145/3341163.3347732

[236] Forner-Cordero A, Mateu-Arce M, Forner-Cordero I, Alcántara E, Moreno JC, Pons JL (2008) Study of the motion artefacts of skin-mounted inertial sensors under different attachment conditions. Physiolo- gical Measurement 29(4):N21-31, https://doi.org/ $10.1088 / 0967-3334 / 29 / 4 / \mathrm{n} 01$

[237] Fouré A, Gondin J (2021) Skeletal Muscle Damage Produced by Electrically Evoked Muscle Contractions. Exercise and Sport Sciences Reviews 49(1):5965, http://doi.org/10.1249/JES.0000000000000239

[238] Freeman D, Reeve S, Robinson A, Ehlers A, Clark D, Spanlang B, Slater M (2017) Virtual reality in the assessment, understanding, and treatment of mental health disorders. Psychological Medicine 47(14):2393-2400, https://doi.org/10.1017/ S003329171700040X

[239] Fregly BJ, Besier TF, Lloyd DG, Delp SL, Banks SA, Pandy MG, D'Lima DD (2012) Grand challenge competition to predict in vivo knee loads. Journal of Orthopaedic Research 30(4):503-513, https://doi.org/ $10.1002 /$ jor. 22023

[240] Freitas SR, Mendes B, Sant GL, Andrade RJ, Nordez A, Milanovic Z (2018) Can chronic stretching change the muscle-tendon mechanical properties? A review. Scandinavian Journal of Medicine \& Science in Sports 28(3):794-806, https://doi.org/10.1111/sms.12957

[241] Freiwald J, Hoppe MW, Javanmardi S, Hotfiel T, Engelhardt M, Grim C, Baumgart C (2020) Strength training-future directions and misconceptions in rehabilitation after knee injuries-Part 1. Sports Orthopaedics and Traumatology https:// doi.org/10.1016/j.orthtr.2020.07.008

[242] Frevel N, Schmidt SL, Beiderbeck D, Penkert B, Subirana B (2020) Taxonomy of Sportstech. In: Schmidt SL (ed) 21st Century Sports: How Technologies Will Change Sports in the Digital Age, Future of Business and Finance, Springer International Publishing, Cham, pp 15-37, https://doi.org/10.1007/ $978-3-030-50801-2 \_2$

[243] di Fronso S, Tamburro G, Robazza C, Bortoli L, Comani S, Bertollo M (2018) Focusing Attention on Muscle Exertion Increases EEG Coherence in an Endurance Cycling Task. Frontiers in Psychology 9, https://doi.org/10.3389/fpsyg.2018.01249

[244] di Fronso S, Fiedler P, Tamburro G, Haueisen J, Bertollo M, Comani S (2019) Dry EEG in Sports Sciences: A Fast and Reliable Tool to Assess Individual Alpha Peak Frequency Changes Induced by Physical Effort. Frontiers in Neuroscience 13, https: //doi.org/10.3389/fnins.2019.00982

[245] Fry AC, Herda TJ, Sterczala AJ, Cooper MA, Andre MJ (2016) Validation of a motion capture system for deriving accurate ground reaction forces without a force plate. Big Data Analytics 1(1):11, https: //doi.org/10.1186/s41044-016-0008-y

[246] Fuglsang EI, Telling AS, Sørensen H (2017) Effect of Ankle Mobility and Segment Ratios on Trunk Lean in the Barbell Back Squat. Journal of Strength and Conditioning Research 31(11):3024-3033, https: //doi.org/10.1519/jsc.0000000000001872

[247] Fujii K, Takeishi N, Kibushi B, Kouzaki M, Kawahara Y (2019) Data-driven spectral analysis for coordinative structures in periodic human locomotion. Scientific Reports 9(1):16755, https:// doi.org/10.1038/s41598-019-53187-1

[248] Fujii K, Takeishi N, Hojo M, Inaba Y, Kawahara Y (2020) Physically-interpretable classification of biological network dynamics for complex collective motions. arXiv:190504859 [cs, math, stat] http: //arxiv.org/abs/1905.04859

[249] Fujita RA, Villalba MM, Silva NRS, Pacheco MM, Gomes MM (2020) Mind-Muscle Connection: Verbal Instructions Alter Electromyographic Activity for Elbow Flexors and Extensors During Co-Contraction Training. Perceptual and Motor Skills p 0031512520949089, https://doi.org/ $10.1177 / 0031512520949089$ 
[250] Funk C, Nagendra S, Scott J, Ravichandran B, Challis JH, Collins RT, Liu Y (2019) Learning Dynamics from Kinematics: Estimating 2D Foot Pressure Maps from Video Frames. arXiv:181112607 [cs] http: //arxiv.org/abs/1811.12607

[251] Fursin G (2020) The Collective Knowledge project: making ML models more portable and reproducible with open APIs, reusable best practices and MLOps. arXiv:200607161 [csLG] https: //arxiv.org/ $\mathrm{abs} / 2006.07161 \mathrm{v} 2$

[252] Galbusera F, Cina A, Panico M, Albano D, Messina C (2020) Image-based biomechanical models of the musculoskeletal system. European Radiology Experimental 4(1):49, https://doi.org/10.1186/s41747$020-00172-3$

[253] Gambhir SS, Ge TJ, Vermesh O, Spitler R (2018) Toward achieving precision health. Science Translational Medicine 10(430), http://doi.org/10.1126/ scitranslmed.aao3612

[254] Gámez Díaz R, Yu Q, Ding Y, Laamarti F, El Saddik A (2020) Digital Twin Coaching for Physical Activities: A Survey. Sensors 20(20):5936, https: //doi.org/10.3390/s20205936

[255] Gao W, Emaminejad S, Nyein HYY, Challa S, Chen K, Peck A, Fahad HM, Ota H, Shiraki H, Kiriya D, Lien DH, Brooks GA, Davis RW, Javey A (2016) Fully integrated wearable sensor arrays for multiplexed in situ perspiration analysis. Nature 529(7587):509-514, https://doi.org/ 10.1038 /nature 16521

[256] García-Pinillos F, Jaén-Carrillo D, Hermoso VS, Román PL, Delgado P, Martinez C, Carton A, Seruendo LR (2020) Agreement Between Spatiotemporal Gait Parameters Measured by a Markerless Motion Capture System and Two Reference Systems-a Treadmill-Based Photoelectric Cell and High-Speed Video Analyses: Comparative Study. JMIR mHealth and uHealth 8(10):e19498, https: //doi.org/10.2196/19498

[257] Garcia-Retortillo S, Rizzo R, Wang JWJL, Sitges C, Ivanov PC (2020) Universal spectral profile and dynamic evolution of muscle activation: a hallmark of muscle type and physiological state. Journal of Applied Physiology 129(3):419-441, https://doi.org/ 10.1152/japplphysiol.00385.2020

[258] Gaube S, Suresh H, Raue M, Merritt A, Berkowitz SJ, Lermer E, Coughlin JF, Guttag JV, Colak E, Ghassemi M (2021) Do as AI say: susceptibility in deployment of clinical decision-aids. npj Digital Medicine 4(1):1-8, https://doi.org/10.1038/ s41746-021-00385-9

[259] Gaver W (2012) What should we expect from research through design? In: Proceedings of the SIGCHI Conference on Human Factors in Computing Systems, Association for Computing Machinery, New York, NY, USA, CHI '12, pp 937-946, https://doi.org/ $10.1145 / 2207676.2208538$

[260] Genç Ç, Erkaya M, Balcı F, Özcan O (2018) Exploring Dynamic Expressions on Soft Wearables for Physical Exercises. In: Proceedings of the 2018 ACM Conference Companion Publication on Designing Interactive Systems, Association for Computing Machinery, New York, NY, USA, DIS '18 Companion, pp 147-152, https://doi.org/10.1145/3197391.3205427

[261] Gene-Morales J, Flandez J, Juesas A, Gargallo P, Miñana I, Colado JC (2020) A systematic review on the muscular activation on the lower limbs with five different variations of the squat exercise. Journal of Human Sport and Exercise 15(Proc4):S1277-S1299, http://doi.org/10.14198/jhse.2020.15.Proc4.28

[262] Geng W, Cuthbert TJ, Menon C (2021) Conductive Thermoplastic Elastomer Composite Capacitive Strain Sensors and Their Application in a Wear- able Device for Quantitative Joint Angle Prediction. ACS Applied Polymer Materials 3(1):122-129, https://doi.org/10.1021/acsapm.0c00708

[263] Gerry L, Ens B, Drogemuller A, Thomas B, Billinghurst M (2018) Levity: A Virtual Reality System that Responds to Cognitive Load. In: Extended Abstracts of the 2018 CHI Conference on Human Factors in Computing Systems, Association for Computing Machinery, New York, NY, USA, CHI EA '18, pp 1-6, https://doi.org/10.1145/3170427.3188479

[264] Ghadiyaram D, Feiszli M, Tran D, Yan X, Wang H, Mahajan D (2019) Large-scale weakly-supervised pretraining for video action recognition. arXiv:190500561 [cs] http://arxiv.org/abs/1905.00561

[265] Ghaffari R, Rogers JA, Ray TR (2021) Recent progress, challenges, and opportunities for wearable biochemical sensors for sweat analysis. Sensors and Actuators B: Chemical 332:129447, https://doi.org/ $10.1016 / j . s n b .2021 .129447$

[266] Ghandeharioun A, Eoff B, Jou B, Picard RW (2019) Characterizing Sources of Uncertainty to Proxy Calibration and Disambiguate Annotator and Data Bias. arXiv:190909285 [cs, stat] http://arxiv.org/abs/ 1909.09285

[267] Ghorbani S, Mahdaviani K, Thaler A, Kording K, Cook DJ, Blohm G, Troje NF (2020) MoVi: A Large Multipurpose Motion and Video Dataset. arXiv:200301888 [cs, eess] http://arxiv.org/abs/ 2003.01888

[268] Glaeser C (2017) A Buyer's Guide for Athlete Management System Software. https: //simplifaster.com/articles/buyers - guideathlete-management-system-software/

[269] Glazier PS (2017) Towards a Grand Unified Theory of sports performance. Human Movement Science 56:139-156, https://doi.org/10.1016/ j.humov. 2015.08.001

[270] Gomes J, Neto T, Vaz JR, Schoenfeld BJ, Freitas SR (2020) Is there a relationship between back squat depth, ankle flexibility, and Achilles tendon stiffness? Sports Biomechanics 0(0):1-14, http:// doi.org/10.1080/14763141.2019.1690569

[271] Gómez-Carmona P, Fernández-Cuevas I, SilleroQuintana M, Arnaiz-Lastras J, Navandar A (2020) Infrared Thermography Protocol on Reducing the Incidence of Soccer Injuries. Journal of Sport Rehabilitation 29(8):1222-1227, https://doi.org/10.1123/ jsr.2019-0056

[272] Gonzalez AM, Mangine GT, Spitz RW, Ghigiarelli JJ, Sell KM (2019) Agreement between the Open Barbell and Tendo Linear Position Transducers for Monitoring Barbell Velocity during Resistance Exercise. Sports 7(5):125, https://doi.org/10.3390/ sports 7050125

[273] González-Badillo JJ, Sánchez-Medina L (2010) Movement velocity as a measure of loading intensity in resistance training. International Journal of Sports Medicine 31(5):347-352

[274] González-Sales M, Barrière O, Tremblay PO, Nekka F, Desrochers J, Tanguay M (2016) Modeling Testosterone Circadian Rhythm in Hypogonadal Males: Effect of Age and Circannual Variations. The AAPS Journal 18(1):217-227, https://doi.org/ $10.1208 / \mathrm{s} 12248-015-9841-6$

[275] Greenhalgh T (1999) Narrative based medicine in an evidence based world. BMJ 318(7179):323-325, https://doi.org/10.1136/bmj.318.7179.323

[276] Greig L, Stephens Hemingway BH, Aspe RR, Cooper K, Comfort P, Swinton PA (2020) Autoregulation in Resistance Training: Addressing the Inconsistencies. Sports Medicine https://doi.org/10.1007/s40279020-01330-8

[277] Grgic J, Lazinica B, Garofolini A, Schoenfeld BJ, 
Saner NJ, Mikulic P (2019) The effects of time of day-specific resistance training on adaptations in skeletal muscle hypertrophy and muscle strength: A systematic review and meta-analysis. Chronobiology International 36(4):449-460, https://doi.org/ 10.1080/07420528.2019.1567524

[278] Grill JB, Strub F, Altché F, Tallec C, Richemond PH, Buchatskaya E, Doersch C, Pires BA, Guo ZD, Azar MG, Piot B, Kavukcuoglu K, Munos R, Valko M (2020) Bootstrap your own latent: A new approach to self-supervised Learning. arXiv:200607733 [cs, stat] http://arxiv.org/abs/2006.07733

[279] Grønsund T, Aanestad M (2020) Augmenting the algorithm: Emerging human-in-the-loop work configurations. The Journal of Strategic Information Systems 29(2):101614, https://doi.org/10.1016/ j.jsis.2020.101614

[280] Gronwald T, Törpel A, Herold F, Budde H (2020) Perspective of Dose and Response for Individualized Physical Exercise and Training Prescription. Journal of Functional Morphology and Kinesiology 5(3):48, https://doi.org/10.3390/jfmk5030048

[281] Grooms DR, Diekfuss JA, Ellis JD, Yuan W, Dudley J, Foss KDB, Thomas S, Altaye M, Haas L, Williams B, Lanier JM, Bridgewater K, Myer GD (2019) A Novel Approach to Evaluate Brain Activation for Lower Extremity Motor Control. Journal of Neuroimaging 29(5):580-588, https://doi.org/ $10.1111 / j$ on. 12645

[282] Grudinschi M, Norland K, Lee SW, Lim S (2020) Task Analysis on Yoga Poses Toward a Wearable Sensorbased Learning System for Users with Visual Impairment. Tech. rep., Smart Life In Motion (SLIM) Lab | University of Arizona

[283] Guo C, Ashrafian H, Ghafur S, Fontana G, Gardner C, Prime M (2020) Challenges for the evaluation of digital health solutions-A call for innovative evidence generation approaches. npj Digital Medicine 3(1):114, https://doi.org/10.1038/s41746-020-00314-2

[284] Guo J, Zhou B, Yang C, Dai Q, Kong L (2019) Stretchable and Temperature-Sensitive Polymer Optical Fibers for Wearable Health Monitoring. Advanced Functional Materials 29(33):1902898, https: //doi.org/10.1002/adfm.201902898

[285] Guo JY, Zheng YP, Xie HB, Chen X (2010) Continuous monitoring of electromyography (EMG), mechanomyography (MMG), sonomyography (SMG) and torque output during ramp and step isometric contractions. Medical Engineering \& Physics 32(9):1032-1042, https: //doi.org/10.1016/j.medengphy.2010.07.004

[286] Guo L, Sandsjö L, Ortiz-Catalan M, Skrifvars M (2020) Systematic review of textilebased electrodes for long-term and continuous surface electromyography recording. Textile Research Journal 90(2):227-244, https://doi.org/10.1177/0040517519858768

[287] Gupta L, Morgan K, North C, Gilchrist S (2020) Napping in high-performance athletes: Sleepiness or sleepability? European Journal of Sport Science 0(0):1-10, https://doi.org/10.1080/ 17461391.2020 .1743765

[288] Gupta SK, Lindemulder EA, Sathyan G (2000) Modeling of Circadian Testosterone in Healthy Men and Hypogonadal Men. The Journal of Clinical Pharmacology 40(7):731-738, https://doi.org/10.1177/ 00912700022009486

[289] Gwak D, Sim G, Poli M, Massaroli S, Choo J, Choi E (2020) Neural Ordinary Differential Equations for Intervention Modeling. arXiv:201008304 [cs] http:// arxiv.org/abs/2010.08304

[290] Habiba M, Pearlmutter BA (2020) Neural ODEs for Informative Missingness in Multivariate Time
Series. arXiv:200510693 [cs, stat] http://arxiv.org/ abs/2005.10693

[291] Hadsell R, Rao D, Rusu AA, Pascanu R (2020) Embracing Change: Continual Learning in Deep Neural Networks. Trends in Cognitive Sciences 24(12):10281040, https://doi.org/10.1016/j.tics.2020.09.004

[292] Haghighi Osgouei R, Soulsby D, Bello F (2019) Rehabilitation exergames: use of motion sensing and machine learning to quantify exercise performance in healthy volunteers. JMIR Rehabil Assist Technol 7(2):e17289, https://doi.org/10.2196/17289

[293] Hakim H, Puel F, Bertucci W (2020) Injury assessment in circus student-artists population; preliminary study. Science \& Sports 35(3):154-160, https://www.sciencedirect.com/science/article/ pii/S0765159719301601

[294] Hakim T, Shimshoni I (2020) A-MAL: Automatic Movement Assessment Learning from Properly Performed Movements in 3D Skeleton Videos. arXiv:190710004 [cs] http: //arxiv.org/abs/1907.10004

[295] Halperin I, Emanuel A (2020) Rating of Perceived Effort: Methodological Concerns and Future Directions. Sports Medicine 50(4):679-687, https:// doi.org/10.1007/s40279-019-01229-z

[296] Halperin I, Vigotsky AD, Foster C, Pyne DB (2017) Strengthening the Practice of Exercise and SportScience Research. International Journal of Sports Physiology and Performance 13(2):127-134, https: //doi.org/10.1123/ijspp.2017-0322

[297] Halson SL (2014) Monitoring Training Load to Understand Fatigue in Athletes. Sports Medicine 44(2):139-147, https://doi.org/10.1007/s40279$014-0253-z$

[298] Han F, Reily B, Hoff W, Zhang H (2017) Spacetime representation of people based on $3 \mathrm{D}$ skeletal data: A review. Computer Vision and Image Understanding 158:85-105, https://doi.org/10.1016/ j.cviu.2017.01.011

[299] Han PKJ, Strout TD, Gutheil C, Germann C, King B, Ofstad E, Gulbrandsen P, Trowbridge R (2021) How Physicians Manage Medical Uncertainty: A Qualitative Study and Conceptual Taxonomy. Medical Decision Making p 0272989X21992340, https: //doi.org/10.1177/0272989x21992340

[300] Han S, Liu B, Wang R, Ye Y, Twigg CD, Kin K (2018) Online optical marker-based hand tracking with deep labels. ACM Transactions on Graphics 37(4):1-10, https://doi.org/10.1145/3197517.3201399

[301] Hannay KM, Moreno JP (2020) Integrating wearable data into circadian models. Current Opinion in Systems Biology 22:32-38, https://doi.org/10.1016/ j.coisb.2020.08.001

[302] Harper SE, Roembke RA, Zunker JD, Thelen DG, Adamczyk PG (2020) Wearable Tendon Kinetics. Sensors 20(17):4805, https://doi.org/10.3390/ s20174805

[303] Harty PS, Sieglinger B, Heymsfield SB, Shepherd JA, Bruner D, Stratton MT, Tinsley GM (2020) Novel body fat estimation using machine learning and 3dimensional optical imaging. European Journal of Clinical Nutrition 74(5):842-845, https://doi.org/ $10.1038 / \mathrm{s} 41430-020-0603-\mathrm{x}$

[304] Harvey H, Karpati E, Khara G, Korkinof D, Ng A, Austin C, Rijken T, Kecskemethy P (2019) The Role of Deep Learning in Breast Screening. Current Breast Cancer Reports 11(1):17-22, https:// doi.org/10.1007/s12609-019-0301-7

[305] Hasselberg MJ, McMahon J, Parker K (2013) The validity, reliability, and utility of the iButton $\mathbb{R}$ for measurement of body temperature circadian rhythms in sleep/wake research. Sleep Medicine 14(1):5-11, https://doi.org/10.1016/j.sleep.2010.12.011 
[306] Hastings MH, Maywood ES, Brancaccio M (2018) Generation of circadian rhythms in the suprachiasmatic nucleus. Nature Reviews Neuroscience 19(8):453-469, https: //doi.org/10.1038/s41583-018-0026-z

[307] Haugen T, Buchheit M (2016) Sprint Running Performance Monitoring: Methodological and Practical Considerations. Sports Medicine (Auckland, NZ) 46(5):641-656, https://doi.org/10.1007/ s40279-015-0446-0

[308] Haun CT, Vann CG, Roberts BM, Vigotsky AD, Schoenfeld BJ, Roberts MD (2019) A Critical Evaluation of the Biological Construct Skeletal Muscle Hypertrophy: Size Matters but So Does the Measurement. Frontiers in Physiology 10, https://doi.org/ 10.3389/f phys. 2019.00247

[309] Hayes LD, Bickerstaff GF, Baker JS (2010) Interactions of Cortisol, Testosterone, and Resistance Training: Influence of Circadian Rhythms. Chronobiology International 27(4):675-705, https://doi.org/ 10.3109/07420521003778773

[310] He X, Chen H, An B (2020) Learning Behaviors with Uncertain Human Feedback. arXiv:200604201 [cs] http://arxiv.org/abs/2006.04201

[311] Hedayatpour N, Falla D (2015) Physiological and Neural Adaptations to Eccentric Exercise: Mechanisms and Considerations for Training. https:// doi.org/10.1155/2015/193741

[312] Heil N (2020) Silicon Valley Thinks Artificial Intelligence Can Upgrade Your Workouts. Men's Health https://www.menshealth.com/fitness/a33957101/ artificial-intelligence-workout /

[313] Hekler E, Tiro JA, Hunter CM, Nebeker C (2020) Precision Health: The Role of the Social and Behavioral Sciences in Advancing the Vision. Annals of Behavioral Medicine 54(11):805-826, https://doi.org/ $10.1093 / \mathrm{abm} / \mathrm{kaaa0} 18$

[314] Helms ER, Isuf O (2019) How Technology Can Make Lifting Easier. https://ironculture.libsyn.com/ep18-how-technology-can-make-lifting-eas ier

[315] Helms ER, Isuf O (2019) Pain Science, Injury \& Rehab. https://ironculture.libsyn.com/ep-33-painscience-injury-rehab

[316] Helms ER, Isuf O (2020) Getting Stronger By Understanding Your Biomechanics (ft. Megan Bryanton-Jones \& Andrew Vigotsky). https://ironculture.libsyn.com/ep-67-gettingstronger-by - unders tanding-your-biomechanicsft-megan-bryant on - jones-andrew-vigotsky

[317] Helms ER, Isuf O (2020) Strength \& Conditioning (for Mixed Martial Arts). https: //ironculture.libsyn.com/ep-76-strengthconditioning-for-mixed-martial-arts

[318] Helms ER, Isuf O (2020) Training Differences In Men \& Women. https://ironculture.libsyn.com/ep-52training-differences-in-men-women

[319] Helms ER, Isuf O (2021) What Hardcore Lifting Gets Wrong. https://ironculture.libsyn.com/ep106 -what-hardcore-lifters-get-wrong

[320] Helms ER, Storey A, Cross MR, Brown SR, Lenetsky S, Ramsay H, Dillen C, Zourdos MC (2017) RPE and Velocity Relationships for the Back Squat, Bench Press, and Deadlift in Powerlifters. Journal of Strength and Conditioning Research 31(2):292-297, https://doi.org/10.1519/jsc.0000000000001517

[321] Helms ER, Byrnes RK, Cooke DM, Haischer MH, Carzoli JP, Johnson TK, Cross MR, Cronin JB, Storey AG, Zourdos MC (2018) RPE vs. Percentage 1RM Loading in Periodized Programs Matched for Sets and Repetitions. Frontiers in Physiology 9, https://doi.org/10.3389/fphys.2018.00247

[322] Helms ER, Kwan K, Sousa CA, Cronin JB, Storey AG, Zourdos MC (2020) Methods for Regulating and Monitoring Resistance Training. Journal of $\mathrm{Hu}-$ man Kinetics 74(1):23-42, https://doi.org/10.2478/ hukin-2020-0011

[323] Hendry D, Leadbetter R, McKee K, Hopper L, Wild C, O'Sullivan P, Straker L, Campbell A (2020) An Exploration of Machine-Learning Estimation of Ground Reaction Force from Wearable Sensor Data. Sensors 20(3):740, https://doi.org/10.3390/s20030740

[324] Herold F, Müller P, Gronwald T, Müller NG (2019) Dose-Response Matters! - A Perspective on the Exercise Prescription in Exercise-Cognition Research. Frontiers in Psychology 10, https://dx.doi.org/ 10.3389/fpsyg. 2019.02338

[325] Herold F, Gronwald T, Scholkmann F, Zohdi H, Wyser D, Müller NG, Hamacher D (2020) New Directions in Exercise Prescription: Is There a Role for Brain-Derived Parameters Obtained by Functional Near-Infrared Spectroscopy? Brain Sciences 10(6):342, https://doi.org/10.3390/ brainsci10060342

[326] Herold F, Hamacher D, Törpel A, Goldschmidt L, Müller NG, Schega L (2020) Does squatting need attention?-A dual-task study on cognitive resources in resistance exercise. PLOS ONE 15(1):e0226431, http://doi.org/10.1371/journal.pone.0226431

[327] Herold F, Törpel A, Hamacher D, Budde H, Gronwald T (2020) A Discussion on Different Approaches for Prescribing Physical Interventions - Four Roads Lead to Rome, but Which One Should We Choose? Journal of Personalized Medicine 10(3):55, https: //doi.org/10.3390/jpm10030055

[328] Herold JL (2021) Mathematical modeling, simulation, and optimization of loading schemes for isometric resistance training. PhD Thesis, University of Heidelberg, Heidelberg, Germany, https://doi.org/ 10.11588/heidok.00029542

[329] Herold JL, Sommer A (2020) A mathematical model-based approach to optimize loading schemes of isometric resistance training sessions. bioRxiv p 2020.04.16.044578, https://doi.org/ 10.1101/2020.04.16.044578

[330] Hesse J, Malhan D, Yal?in M, Aboumanify O, Basti A, Relógio A (2020) An Optimal Time for Treatment-Predicting Circadian Time by Machine Learning and Mathematical Modelling. Cancers 12(11):3103, https://doi.org/10.3390/ cancers 12113103

[331] Heydari MJ, Shiry Ghidary S (2019) Cross-modal motion regeneration using Multimodal Deep Belief Network. Journal of Visual Communication and Image Representation 58:245-260, https://doi.org/ 10.1016/j.jvcir.2018.11.042

[332] Heyen NB (2020) From self-tracking to selfexpertise: The production of self-related knowledge by doing personal science. Public Understanding of Science 29(2):124-138, https://doi.org/10.1177/ 0963662519888757

[333] Hiasa Y, Otake Y, Takao M, Ogawa T, Sugano N, Sato Y (2019) Automated Muscle Segmentation from Clinical CT using Bayesian U-Net for Personalized Musculoskeletal Modeling. arXiv:190708915 [cs, eess] http://arxiv.org/abs/1907.08915

[334] Hindle BR, Lorimer A, Winwood P, Keogh JWL (2020) A systematic review of the biomechanical research methods used in strongman studies. Sports Biomechanics 19(1):90-119, https://doi.org/ $10.1080 / 14763141.2019 .1598480$

[335] Hippocrate AAE, Luhanga ET, Masashi T, Watanabe K, Yasumoto K (2017) Smart gyms need smart mirrors: design of a smart gym concept through contextual inquiry. In: Proceedings of the 2017 ACM International Joint Conference on Pervasive and Ubiquitous Computing and Proceedings 
of the 2017 ACM International Symposium on Wearable Computers, Association for Computing Machinery, New York, NY, USA, UbiComp '17, pp 658-661, https://doi.org/10.1145/3123024.3124427

[336] Hooker S, Erhan D, Kindermans PJ, Kim B (2019) A Benchmark for Interpretability Methods in Deep Neural Networks. In: Wallach H, Larochelle H, Beygelzimer A, Alché-Buc Fd, Fox E, Garnett R (eds) Advances in Neural Information Processing Systems 32, Curran Associates, Inc., pp 9737-9748, https: //arxiv.org/abs/1806.10758

[337] Hooper DR, Kraemer WJ, Focht BC, Volek JS, DuPont WH, Caldwell LK, Maresh CM (2017) Endocrinological Roles for Testosterone in Resistance Exercise Responses and Adaptations. Sports Medicine 47(9):1709-1720, https://doi.org/10.1007/s40279$017-0698-y$

[338] Horschig DA, Sonthana DK, Neff $T$ (2017) The Squat Bible: The Ultimate Guide to Mastering the Squat and Finding Your True Strength, 1st edn. CreateSpace Independent Publishing Platform

[339] Horst F, Lapuschkin S, Samek W, Müller KR, Schöllhorn WI (2019) Explaining the unique nature of individual gait patterns with deep learning. Scientific Reports 9(1):2391, https://doi.org/10.1038/s41598019-38748-8

[340] Horst F, Janssen D, Beckmann H, Schöllhorn WI (2020) Can Individual Movement Characteristics Across Different Throwing Disciplines Be Identified in High-Performance Decathletes? Frontiers in Psychology 11, https://dx.doi.org/10.3389/ fpsyg.2020.02262

[341] Hou Y, Liu L, Chen X, Li Q, Li J (2020) Association between circadian disruption and diseases: A narrative review. Life Sciences 262:118512, https: //doi.org/10.1016/j.Ifs.2020.118512

[342] Houcke JV, Galibarov PE, Acker GV, Fauconnier S, Allaert E, Hoof TV, Almeida DF, Steenackers G, Pattyn C, Audenaert EA (2020) Personalized hip joint kinetics during deep squatting in young, athletic adults. Computer Methods in Biomechanics and Biomedical Engineering 23(1):23-32, https://doi.org/ $10.1080 / 10255842.2019 .1699539$

[343] Houy N (2020) Optimizing training programs for athletic performance: a Monte-Carlo Tree Search variant method. medRxiv p 2020.10.31.20223768, https: //doi.org/10.1101/2020.10.31.20223768

[344] Hsia J (2020) Prevalence and Localization of Injuries and Pain in Swedish Bodybuliding and Fitness Athletes. PhD thesis, Umea University, http://urn.kb.se/resolve?urn=urn:nbn: se: umu: diva-177102

[345] Huang F, Zeng A, Liu M, Lai Q, Xu Q (2020) DeepFuse: An IMU-Aware Network for Real-Time 3D Human Pose Estimation from Multi-View Image. In: Proceedings of the IEEE/CVF Winter Conference on Applications of Computer Vision, pp 429-438, https: //openaccess.thecvf.com/content_WACV_2020/html/ Huang_DeepFuse_An_IMU-Aware_Network_for_Real Time_3D_Human_Pose_Estimation_WACV_2020_paper.h

[346] Huang X, Rezaei B, Ostadabbas S (2019) AH-CoLT: an AI-Human Co-Labeling Toolbox to Augment Efficient Groundtruth Generation. In: 2019 IEEE 29th International Workshop on Machine Learning for Signal Processing (MLSP), pp 1-6, https://doi.org/ 10.1109/MLSP. 2019.8918924

[347] Hughes RL (2020) A Review of the Role of the Gut Microbiome in Personalized Sports Nutrition. Frontiers in Nutrition 6, https://doi.org/10.3389/ fnut.2019.00191

[348] Hughes T, Riley RD, Callaghan MJ, Sergeant JC (2020) The Value of Preseason Screening for Injury Prediction: The Development and Internal Valid- ation of a Multivariable Prognostic Model to Predict Indirect Muscle Injury Risk in Elite Football (Soccer) Players. Sports Medicine - Open 6(1):22, https://doi.org/10.1186/s40798-020-00249-8

[349] Hülsmann F, Göpfert JP, Hammer B, Kopp S, Botsch M (2018) Classification of motor errors to provide real-time feedback for sports coaching in virtual reality - A case study in squats and Tai Chi pushes. Computers \& Graphics 76:47-59, https://doi.org/ 10.1016/j.cag.2018.08.003

[350] Hwang DH, Aso K, Yuan Y, Kitani K, Koike H (2020) MonoEye: Multimodal Human Motion Capture System Using A Single Ultra-Wide Fisheye Camera. In: Proceedings of the 33rd Annual ACM Symposium on User Interface Software and Technology, Association for Computing Machinery, New York, NY, USA, UIST '20, pp 98-111, https://doi.org/ $10.1145 / 3379337.3415856$

[351] Iellamo F, Lucini D, Volterrani M, Casasco M, Salvati A, Gianfelici A, Gianfrancesco AD, Urso A, Manzi V (2019) Autonomic nervous system responses to strength training in top-level weight lifters. Physiological Reports 7(20):e14233, https:// doi.org/10.14814/phy2.14233

[352] Ijaz K, Ahmadpour N, Wang Y, Calvo RA (2020) Player Experience of Needs Satisfaction (PENS) in an Immersive Virtual Reality Exercise Platform Describes Motivation and Enjoyment. International Journal of Human-Computer Interaction 36(13):1195-1204, https://doi.org/10.1080/ 10447318.2020.1726107

[353] Imani S, Bandodkar AJ, Mohan AMV, Kumar R, Yu S, Wang J, Mercier PP (2016) A wearable chemical-electrophysiological hybrid biosensing system for real-time health and fitness monitoring. Nature Communications 7(1):11650, https://doi.org/10.1038/ ncomms 11650

[354] Imani Nejad Z, Khalili K, Hosseini Nasab SH, Schütz P, Damm P, Trepczynski A, Taylor WR, Smith CR (2020) The Capacity of Generic Musculoskeletal Simulations to Predict Knee Joint Loading Using the CAMS-Knee Datasets. Annals of Biomedical Engineering 48(4):1430-1440, https://doi.org/10.1007/ s10439-020-02465-5

[355] Impellizzeri FM, Marcora SM, Coutts AJ (2019) Internal and External Training Load: 15 Years On. International Journal of Sports Physiology and Performance 14(2):270-273, https://doi.org/10.1123/ ijspp.2018-0935

[356] Impellizzeri FM, McCall A, Ward P, Bornn L, Coutts AJ (2020) Training Load and Its Role in Injury Prevention, Part 2: Conceptual and Methodologic Pitfalls. Journal of Athletic Training 55(9):893-901, https://doi.org/10.4085/1062-6050-501-19

[357] Impellizzeri FM, Menaspà P, Coutts AJ, Kalkhoven J, Menaspà MJ (2020) Training Load and Its Role in Injury Prevention, Part I: Back to the Future. Journal of Athletic Training 55(9):885-892, https://doi.org/ 10.4085/1062-6050-500-19

[358] Impellizzeri FM, Tenan MS, Kempton T, Novak A, Coutts AJ (2020) Acute:Chronic Workload Ratio: Conceptual Issues and Fundamental Pitfalls. International Journal of Sports Physiology and Performance 15(6):907-913, https://doi.org/10.1123/ ijspp.2019-0864

[359] Institute W (2020) Ever Active: Strength Tracking Sensor for Sustainable Personalized Training. https://wyss.harvard.edu/technology/ everactive-strength-tracking-sensor-forsustainable-personalized-training/

[360] Ishii S, Nkurikiyeyezu K, Yokokubo A, Lopez G (2020) ExerSense: Real-Tme Physical Exercise Segmentation, Classification, and Counting Algorithm 
Using an IMU Sensor. arXiv:200410026 [cs] http: //arxiv.org/abs/2004.10026

[361] Islam H, Gurd BJ (2020) Exercise response variability: Random error or true differences in exercise response? Experimental Physiology 105(12):20222024, https://doi.org/10.1113/ep089015

[362] Islam H, Bonafiglia JT, Del Giudice M, Pathmarajan R, Simpson CA, Quadrilatero J, Gurd BJ (2020) Repeatability of training-induced skeletal muscle adaptations in active young males. Journal of Science and Medicine in Sport https://doi.org/10.1016/ j.j. jams.2020.10.016

[363] Israetel M (2020) Should You Test Your 1 Rep Max For Hypertrophy? https://youtu.be/4luBPhK-rIE

[364] Israetel M, Davis M, Hoffman J, Feather J (2020) Scientific Principles of Hypertrophy Training, 1st edn. Renaissance Periodization, LLC, https://renaissanceperiodization.com/thescientific-principles - of -hypertrophy - training

[365] Izzicupo P, Di Baldassarre A, Ghinassi B, Reichert FF, Kokubun E, Nakamura FY (2019) Can OffTraining Physical Behaviors Influence Recovery in Athletes? A Scoping Review. Frontiers in Physiology 10, https://doi.org/10.3389/fphys.2019.00448

[366] Jain M, Lahlou S, Nekoei H, Butoi V, Bertin P, Rector-Brooks J, Korablyov M, Bengio Y (2021) DEUP: Direct Epistemic Uncertainty Prediction. arXiv:210208501 [cs, stat] http://arxiv.org/abs/ 2102.08501

[367] James LP, Haff GG, Kelly VG, Beckman EM (2016) Towards a Determination of the Physiological Characteristics Distinguishing Successful Mixed Martial Arts Athletes: A Systematic Review of Combat Sport Literature. Sports Medicine 46(10):1525-1551, https://doi.org/10.1007/s40279-016-0493-1

[368] Javitt NP, Raphael SY (2020) Insole to aid in gait stability. https://patents.google.com/patent/ US10595749B1/en

[369] Jeffries AC, Wallace L, Coutts AJ, McLaren SJ, McCall A, Impellizzeri FM (2020) Athlete-Reported Outcome Measures for Monitoring Training Responses: A Systematic Review of Risk of Bias and Measurement Property Quality According to the COSMIN Guidelines. International Journal of Sports Physiology and Performance 15(9):1203-1215, https : //doi.org/10.1123/ijspp.2020-0386

[370] Jenni S, Favaro P (2020) Self-Supervised Multi-View Synchronization Learning for 3D Pose Estimation. In: Proceedings of the Asian Conference on Computer Vision, https://arxiv.org/abs/2010.06218

[371] Jennings N, Jain S (2019) MirrorMatch: Real-Time Detection of Repetitive Movements using Smartphone Camera. In: 2019 IEEE/ACM International Conference on Connected Health: Applications, Systems and Engineering Technologies (CHASE), pp 34, https://doi.org/10.1109/CHASE48038.2019.00007

[372] Jiang W, Xue H, Miao C, Wang S, Lin S, Tian C, Murali S, Hu H, Sun Z, Su L (2020) Towards 3D human pose construction using wifi. In: Proceedings of the 26th Annual International Conference on Mobile Computing and Networking, Association for Computing Machinery, New York, NY, USA, MobiCom '20, pp 1-14, https://doi.org/10.1145/3372224.3380900

[373] Jimenez-Olmedo JM, Penichet-Tomás A, VillalónGasch L, Pueo B (2020) Validity and reliability of smartphone high-speed camera and Kinovea for velocity-based training measurement. Journal of $\mathrm{Hu}-$ man Sport and Exercise https://doi.org/10.14198/ jhse.2021.164.11

[374] Joffe SA, Tallent J (2020) Neuromuscular predictors of competition performance in advanced international female weightlifters: a cross-sectional and longitudinal ana- lysis. Journal of Sports Sciences 38(9):985-993, https://doi.org/10.1080/02640414.2020.1737396

[375] Johnson WR, Mian A, Robinson MA, Verheul J, Lloyd DG, Alderson J (2020) Multidimensional ground reaction forces and moments from wearable sensor accelerations via deep learning. IEEE Transactions on Biomedical Engineering pp 1-1, https: //doi.org/10.1109/TBME.2020.3006158

[376] Johnson WR, Mian A, Robinson MA, Verheul J, Lloyd DG, Alderson JA (2020) Multidimensional ground reaction forces and moments from wearable sensor accelerations via deep learning. arXiv:190307221 [cs] http://arxiv.org/abs/ 1903.07221

[377] Jones A, Jones G, Greig N, Bower P, Brown J, Hind K, Francis P (2019) Epidemiology of injury in English Professional Football players: A cohort study. Physical Therapy in Sport 35:18-22, https://doi.org/ 10.1016/j.ptsp.2018.10.011

[378] Jönsson M, Munkhammar T, Norrbrand L, Berg HE (2019) Foot centre of pressure and ground reaction force during quadriceps resistance exercises; a comparison between force plates and a pressure insole system. Journal of Biomechanics 87:206-210, https: //doi.org/10.1016/j.jbiomech.2019.03.004

[379] Jorgenson KW, Phillips SM, Hornberger TA (2020) Identifying the Structural Adaptations that Drive the Mechanical Load-Induced Growth of Skeletal Muscle: A Scoping Review. Cells 9(7):1658, https://doi.org/ $10.3390 /$ cells 9071658

[380] Josephson CB, Wiebe S (2020) Precision Medicine: Academic dreaming or clinical reality? Epilepsia n/a(n/a), https://doi.org/10.1111/epi.16739

[381] Joy JM, Lowery RP, Oliveira de Souza E, Wilson JM (2016) Elastic Bands as a Component of Periodized Resistance Training. Journal of Strength and Conditioning Research 30(8):2100-2106, https://doi.org/ $10.1519 /$ jsc.0b013e3182986bef

[382] Joyce DW, Geddes J (2020) When Deploying Predictive Algorithms, Are Summary Performance Measures Sufficient? JAMA Psychiatry 77(5):447, http: //doi.org/10.1001/jamapsychiatry.2019.4484

[383] Jung YH, Kim JH, Rogers JA (2020) SkinIntegrated Vibrohaptic Interfaces for Virtual and Augmented Reality. Advanced Functional Materials n/a(n/a):2008805, https://doi.org/10.1002/ adfm. 202008805

[384] Kaichi T, Maruyama T, Tada M, Saito H (2020) Resolving Position Ambiguity of IMU-Based Human Pose with a Single RGB Camera. Sensors 20(19):5453, https://doi.org/10.3390/s20195453

[385] Kakavas G, Malliaropoulos N, Pruna R, Maffulli N (2020) Artificial intelligence: A tool for sports trauma prediction. Injury 51:S63-S65, https:// doi.org/10.1016/j.injury.2019.08.033

[386] Kalkhoven JT, Watsford ML, Coutts AJ, Edwards WB, Impellizzeri FM (2021) Training Load and Injury: Causal Pathways and Future Directions. Sports Medicine https://doi.org/10.1007/s40279020-01413-6

[387] Karamanidis K, Epro G (2020) Monitoring muscletendon adaptation over several years of athletic training and competition in elite track and field jumpers. Frontiers in Physioliology 11: 607544, https: //doi.org/10.3389/fphys.2020.607544

[388] Karargyris A, Kashyap S, Lourentzou I, Wu J, Sharma A, Tong M, Abedin S, Beymer D, Mukherjee V, Krupinski EA, Moradi M (2020) Creation and Validation of a Chest X-Ray Dataset with Eye-tracking and Report Dictation for AI Development. arXiv:200907386 [csCV] https://arxiv.org/ abs/2009.07386v3

[389] Karemaker JM (2020) Interpretation of Heart Rate 
Variability: The Art of Looking Through a Keyhole. Frontiers in Neuroscience 14, https://doi.org/ 10.3389 /fnins 2020.609570

[390] Kasovic J, Martin B, Fahs CA (2019) Kinematic Differences Between the Front and Back Squat and Conventional and Sumo Deadlift. The Journal of Strength \& Conditioning Research 33(12):3213-3219, http://doi.org/10.1519/JSC.0000000000003377

[391] Kasovic J, Martin B, Zourdos M, Carzoli J, Fahs C (2020) Agreement Between the Iron Path App and a Linear Position Transducer for Measuring Average Concentric Velocity and Range of Motion of Barbell Exercises. The Journal of Strength and Conditioning Research Publish Ahead of Print, http://doi.org/ 10.1519/JSC.0000000000003574

[392] Kataoka R, Vasenina E, Loenneke J, Buckner SL (2021) Periodization: Variation in the Definition and Discrepancies in Study Design. Sports Medicine (Auckland, NZ) https://doi.org/10.1007/s40279020-01414-5

[393] Kay W, Carreira J, Simonyan K, Zhang B, Hillier C, Vijayanarasimhan S, Viola F, Green T, Back T, Natsev P, Suleyman M, Zisserman A (2017) The Kinetics Human Action Video Dataset. arXiv:170506950 [cs] http://arxiv.org/abs/1705.06950

[394] Kellmann M, Beckmann J (eds) (2017) Sport, Recovery, and Performance: Interdisciplinary Insights. Routledge, Abingdon, Oxon ; New York, NY

[395] Kenville R, Maudrich T, Carius D, Ragert P (2017) Hemodynamic Response Alterations in Sensorimotor Areas as a Function of Barbell Load Levels during Squatting: An fNIRS Study. Frontiers in Human Neuroscience 11, https://doi.org/10.3389/ fnhum.2017.00241

[396] Kenville R, Maudrich T, Maudrich D, Villringer A, Ragert P (2020) Cerebellar Transcranial Direct Current Stimulation Improves Maximum Isometric Force Production during Isometric Barbell Squats. Brain Sciences 10(4):235, https://doi.org/10.3390/ brains ci10040235

[397] Keogh JWL, Winwood PW (2017) The Epidemiology of Injuries Across the Weight-Training Sports. Sports Medicine 47(3):479-501, https://doi.org/10.1007/ s40279-016-0575-0

[398] Kern F, Winter C, Gall D, Käthner I, Pauli P, Latoschik ME (2019) Immersive Virtual Reality and Gamification Within Procedurally Generated Environments to Increase Motivation During Gait Rehabilitation. In: 2019 IEEE Conference on Virtual Reality and 3D User Interfaces (VR), pp 500-509

[399] Kern H, Carraro U (2020) Home-Based Functional Electrical Stimulation of Human Permanent Denervated Muscles: A Narrative Review on Diagnostics, Managements, Results and Byproducts Revisited 2020. Diagnostics 10(8):529, https:// doi.org/10.3390/diagnostics 10080529

[400] Kettunen E, Critchley W, Kari T (2019) Can Digital Coaching Boost Your Performance? - A Qualitative Study among Physically Active People. In: HICSS 2019, http://doi.org/10.24251/HICSS.2019.163

[401] Khan UA, Khan IA, Din A, Jadoon W, Jadoon RN, Khan MA, Khan FG, Khan AN (2020) Towards a Complete Set of Gym Exercises Detection Using Smartphone Sensors. Scientific Programming https: //doi.org/10.1155/2020/6471438

[402] Khot RA, Hjorth L, Mueller F (2020) Shelfie: A Framework for Designing Material Representations of Physical Activity Data. ACM Transactions on Computer-Human Interaction 27(3):14:1-14:52, https://doi.org/10.1145/3379539

[403] Khurana R, Ahuja K, Yu Z, Mankoff J, Harrison C, Goel M (2018) GymCam: Detecting, Recognizing and Tracking Simultaneous Exercises in Uncon- strained Scenes. Proceedings of the ACM on Interactive, Mobile, Wearable and Ubiquitous Technologies 2(4):185:1-185:17, https://doi.org/10.1145/ 3287063

[404] Kidziński Ł, Mohanty SP, Ong C, Hicks JL, Carroll SF, Levine S, Salathé M, Delp SL (2018) Learning to Run challenge: Synthesizing physiologically accurate motion using deep reinforcement learning. arXiv:180400198 [cs] http://arxiv.org/ abs/1804.00198

[405] Kidziński Ł, Yang B, Hicks JL, Rajagopal A, Delp SL, Schwartz MH (2020) Deep neural networks enable quantitative movement analysis using single-camera videos. Nature Communications 11(1):4054, https: //doi.org/10.1038/s41467-020-17807-z

[406] Kiely J (2012) Periodization paradigms in the 21st century: evidence-led or tradition-driven? International Journal of Sports Physiology and Performance $7(3): 242-250$, https://doi.org/10.1123/ ijspp.7.3.242

[407] Killen BA, Brito da Luz S, Lloyd DG, Carleton AD, Zhang J, Besier TF, Saxby DJ (2020) Automated creation and tuning of personalised muscle paths for OpenSim musculoskeletal models of the knee joint. Biomechanics and Modeling in Mechanobiology https://doi.org/10.1007/s10237-020-01398-1

[408] Kim J, Lee J (2020) Plasma MMP-9, TIMP1 , and TGF- $\beta 1$ Responses to Exercise-Induced Muscle Injury. International Journal of Environmental Research and Public Health 17(2):566, https: //doi.org/10.3390/ijerph17020566

[409] Kim J, Park K, Lee J (2020) Myostatin A55T Genotype is Associated with Strength Recovery Following Exercise-Induced Muscle Damage. International Journal of Environmental Research and Public Health 17(13):4900, https://doi.org/10.3390/ ijerph17134900

[410] Kim K, Hong D (2020) Robotic Biceps Exercise Machine: Hardware Using Series Elastic Actuator and Control With Disturbance Observer. IEEE Access 8:12758-12767, https://doi.org/10.1109/ ACCESS.2019.2963101

[411] Kim S, Lee S, Jeong W (2020) EMG Measurement with Textile-Based Electrodes in Different Electrode Sizes and Clothing Pressures for Smart Clothing Design Optimization. Polymers 12(10):2406, https: //doi.org/10.3390/polym12102406

[412] Kinney AL, Besier TF, D'Lima DD, Fregly BJ (2013) Update on Grand Challenge Competition to Predict in Vivo Knee Loads. Journal of Biomechanical Engineering 135(021012), https://doi.org/10.1115/ 1.4023255

[413] Kipp K, Kim H, Wolf WI (2020) Muscle Forces During the Squat, Split Squat, and Step-Up Across a Range of External Loads in College-Aged Men. The Journal of Strength \& Conditioning Research Publish Ahead of Print, https://doi.org/10.1519/ jsc. 0000000000003688

[414] Kirchner F (2020) Robotics and AI: How Technology May Change the Way We Shape Our Bodies and What This Does to the Mind. In: Schmidt SL (ed) 21st Century Sports: How Technologies Will Change Sports in the Digital Age, Future of Business and Finance, Springer International Publishing, Cham, pp 73-89, https://doi.org/10.1007/978-3-030-50801$2 \_5$

[415] Kirk BJC, Trajano GS, Pulverenti TS, Rowe G, Blazevich AJ (2019) Neuromuscular Factors Contributing to Reductions in Muscle Force After Repeated, High-Intensity Muscular Efforts. Frontiers in Physiology 10, https://doi.org/10.3389/ fphys.2019.00783

[416] Kirk C, Clark DR, Langan-Evans C, Morton JP 
(2020) The physical demands of mixed martial arts: A narrative review using the ARMSS model to provide a hierarchy of evidence. Journal of Sports Sciences 38(24):2819-2841, https://doi.org/10.1080/ 02640414.2020 .1802093

[417] Kirsch A (2021) PowerEvaluationBALD: Efficient Evaluation-Oriented Deep (Bayesian) Active Learning with Stochastic Acquisition Functions. arXiv:210103552 [cs, math] http://arxiv.org/abs/2101.03552

[418] Kishishita Y, Das S, Ramirez AV, Thakur C, Tadayon R, Kurita Y (2019) Muscleblazer: Force-Feedback Suit for Immersive Experience. In: 2019 IEEE Conference on Virtual Reality and 3D User Interfaces (VR), pp 1813-1818, https://doi.org/10.1109/ VR.2019.8797962

[419] Kisilewicz A, Madeleine P, Ignasiak Z, Ciszek B, Kawczynski A, Larsen RG (2020) Eccentric Exercise Reduces Upper Trapezius Muscle Stiffness Assessed by Shear Wave Elastography and Myotonometry. Frontiers in Bioengineering and Biotechnology 8, https://dx.doi.org/10.3389/fbioe.2020.00928

[420] Kittel A, Larkin P, Cunningham I, Spittle M (2020) $360^{\circ}$ Virtual Reality: A SWOT Analysis in Comparison to Virtual Reality. Frontiers in Psychology 11, https://dx.doi.org/10.3389/fpsyg.2020.563474

[421] Klein D, Woods D, Klein G, Perry S (2018) EBM: Rationalist Fever Dreams. Journal of Cognitive Engineering and Decision Making 12(3):227-230, https: //doi.org/10.1177/1555343418779677

[422] Knab AM, Nieman DC, Zingaretti LM, Groen AJ, Pugachev A (2020) Proteomic Profiling and Monitoring of Training Distress and Illness in University Swimmers During a 25-Week Competitive Season. Frontiers in Physiology 11, https://dx.doi.org/ 10.3389/fphys. 2020.00373

[423] Kobak D, Linderman GC (2021) Initialization is critical for preserving global data structure in both $t$ -SNE and UMAP. Nature Biotechnology pp 1-2, https://doi.org/10.1038/s41587-020-00809-z

[424] Kocabas M, Karagoz S, Akbas E (2019) SelfSupervised Learning of 3D Human Pose Using MultiView Geometry. In: Proceedings of the IEEE/CVF Conference on Computer Vision and Pattern Recognition, pp 1077-1086, https://arxiv.org/abs/ 1903.02330

[425] Koch AJ, Pereira R, Machado M (2014) The creatine kinase response to resistance exercise. Journal of Musculoskeletal \& Neuronal Interactions 14(1):68-77, https://pubmed.ncbi.nlm.nih.gov/24583542/

[426] Kocur M, Kloss M, Schwind V, Wolff C, Henze N (2020) Flexing Muscles in Virtual Reality: Effects of Avatars' Muscular Appearance on Physical Performance. In: Proceedings of the Annual Symposium on Computer-Human Interaction in Play, Association for Computing Machinery, New York, NY, USA, CHI PLAY '20, pp 193-205, https://doi.org/10.1145/ 3410404.3414261

[427] Kolodyazhniy V, Späti J, Frey S, Götz T, WirzJustice A, Kräuchi K, Cajochen C, Wilhelm FH (2012) An Improved Method for Estimating Human Circadian Phase Derived From Multichannel Ambulatory Monitoring and Artificial Neural Networks. Chronobiology International 29(8):1078-1097, https : //doi.org/10.3109/07420528.2012.700669

[428] Kompa B, Snoek J, Beam AL (2021) Second opinion needed: communicating uncertainty in medical machine learning. npj Digital Medicine 4(1):1-6, https: //doi.org/10.1038/s41746-020-00367-3

[429] Kompf J, Arandjelović O (2016) Understanding and Overcoming the Sticking Point in Resistance Exercise. Sports Medicine 46(6):751-762, https:// doi.org/10.1007/s40279-015-0460-2
[430] Korhonen O, Väyrynen K, Krautwald T, Bilby G, Broers HAT, Giunti G, Isomursu M (2020) DataDriven Personalization of a Physiotherapy Care Pathway: Case Study of Posture Scanning. JMIR Rehabilitation and Assistive Technologies 7(2):e18508, https://doi.org/10.2196/18508

[431] Koskimäki H, Siirtola P, Röning J (2017) MyoGym: introducing an open gym data set for activity recognition collected using myo armband. In: Proceedings of the 2017 ACM International Joint Conference on Pervasive and Ubiquitous Computing and Proceedings of the 2017 ACM International Symposium on Wearable Computers, Association for Computing Machinery, New York, NY, USA, UbiComp '17, pp 537-546, https://doi.org/10.1145/3123024.3124400

[432] Kosorok MR, Laber EB (2019) Precision Medicine. Annual Review of Statistics and Its Application 6(1):263-286, https://doi.org/10.1146/ annurev-statistics-030718-105251

[433] Kostikiadis IN, Methenitis S, Tsoukos A, Veligekas P, Terzis G, Bogdanis GC (2018) The Effect of Short-Term Sport-Specific Strength and Conditioning Training on Physical Fitness of WellTrained Mixed Martial Arts Athletes. Journal of Sports Science \& Medicine 17(3):348-358, https: //www.ncbi.nlm.nih.gov/pubmed/30116107

[434] Koz A (2021) Embracing Adaptation through Pain Science, When to use RPE, Self-efficacy in Coaching, and How to Craft an Effective Warm-up. https://anchor.fm/circsci/episodes/Tendontraining-and-adaptation-in-circus-and-hypermobility-for-performance-and-recovery-ft--Dr-Keith-Baar--Molecular-Biologist-epsmlj

[435] Koz A (2021) Tendon training and adaptation (in circus and hyper mobility) for performance and recover. https://anchor.fm/circsci/episodes/Tendontraining-and-adaptation-in-circus-and-hypermobility-for-performance-and-recovery-ft--Dr-Keith-Baar--Molecular-Biologist-epsmlj

[436] Kozlová S (2018) The Use of Near-Infrared Spectroscopy in the Sport-Scientific Context. Journal of Neurology and Neurological Disorders 4(2)

[437] Kraemer WJ, Ratamess NA, Hymer WC, Nindl BC, Fragala MS (2020) Growth Hormone(s), Testosterone, Insulin-Like Growth Factors, and Cortisol: Roles and Integration for Cellular Development and Growth With Exercise. Frontiers in Endocrinology 11, https: //doi.org/10.3389/fendo.2020.00033

[438] Kräuchi K (2007) The thermophysiological cascade leading to sleep initiation in relation to phase of entrainment. Sleep Medicine Reviews 11(6):439-451, https://doi.org/10.1016/j.smrv.2007.07.001

[439] Kräuchi K, Wirz-Justice A (1994) Circadian rhythm of heat production, heart rate, and skin and core temperature under unmasking conditions in men. American Journal of Physiology-Regulatory, Integrative and Comparative Physiology 267(3):R819-R829, https://doi.org/10.1152/ajpregu.1994.267.3.R819

[440] Kräuchi K, Konieczka K, Roescheisen-Weich C, Gompper B, Hauenstein D, Schoetzau A, Fraenkl S, Flammer J (2014) Diurnal and menstrual cycles in body temperature are regulated differently: A 28-day ambulatory study in healthy women with thermal discomfort of cold extremities and controls. Chronobiology International 31(1):102-113, https://doi.org/ 10.3109/07420528.2013.829482

[441] Kraus K, Schütz E, Taylor WR, Doyscher R (2014) Efficacy of the Functional Movement Screen: A Review. The Journal of Strength \& Conditioning Research 28(12):3571-3584, http://doi.org/10.1519/ JSC. 0000000000000556

[442] Kravitz RL, Schmid CH, Marois M, Wilsey B, Ward D, Hays RD, Duan N, Wang Y, MacDonald S, Jerant 
A, Servadio JL, Haddad D, Sim I (2018) Effect of Mobile Device-Supported Single-Patient Multi-crossover Trials on Treatment of Chronic Musculoskeletal Pain: A Randomized Clinical Trial. JAMA internal medicine 178(10):1368-1377, https://doi.org/10.1001/ jamainternmed.2018.3981

[443] Krawczyk B (2021) How to Use RPE in Your Powerlifting Program. https: //youtu.be/fr_5J_gB3Xk

[444] Kruk Evd, Reijne MM (2018) Accuracy of human motion capture systems for sport applications; state-of-the-art review. European Journal of Sport Science 18(6):806-819, https://doi.org/ $10.1080 / 17461391.2018 .1463397$

[445] Kruse CS, Krowski N, Rodriguez B, Tran L, Vela J, Brooks M (2017) Telehealth and patient satisfaction: a systematic review and narrative analysis. BMJ Open 7(8):e016242, http://dx.doi.org/ 10.1136/bmj open-2017-016242

[446] Ku JP, Hicks JL, Hastie T, Leskovec J, Ré C, Delp SL (2015) The mobilize center: an NIH big data to knowledge center to advance human movement research and improve mobility. Journal of the American Medical Informatics Association 22(6):1120-1125, https : //doi.org/10.1093/jamia/ocv071

[447] Kubo T, Hirayama K, Nakamura N, Higuchi M (2018) Effect of Accommodating Elastic Bands on Mechanical Power Output during Back Squats. Sports 6(4):151, https://doi.org/10.3390/sports6040151

[448] Kubota A, Iqbal T, Shah JA, Riek LD (2019) Activity recognition in manufacturing: The roles of motion capture and sEMG+inertial wearables in detecting fine vs. gross motion. In: 2019 International Conference on Robotics and Automation (ICRA), pp 65336539, https://doi.org/10.1109/ICRA.2019.8793954

[449] Kundu JN, V RM, Patravali J, Radhakrishnan VB (2020) Unsupervised Cross-Dataset Adaptation via Probabilistic Amodal 3D Human Pose Completion. In: Proceedings of the IEEE/CVF Winter Conference on Applications of Computer Vision, pp 469-478, https://doi.org/10.1109/WACV45572.2020.9093577

[450] Kuniszyk-Jóźkowiak W, Jaszczuk J, Czaplicki A, Szyszka P (2019) Variability of shoulder girdle temperature in the initial phase of the snatch in weightlifting. Acta of Bioengineering and Biomechanics Vol. 21(nr 3), http://dx.doi.org/10.5277/ABB01423-2019-02

[451] Kwon H, Tong C, Haresamudram H, Gao Y, Abowd GD, Lane ND, Ploetz T (2020) IMUTube: Automatic Extraction of Virtual on-body Accelerometry from Video for Human Activity Recognition. arXiv:200605675 [cs, eess] http://arxiv.org/ abs $/ 2006.05675$

[452] Labata-Lezaun N, López-de Celis C, LlurdaAlmuzara L, González-Rueda V, Cadellans-Arróniz A, Pérez-Bellmunt A (2020) Correlation between maximal radial muscle displacement and stiffness in gastrocnemius muscle. Physiological Measurement 41(12):125013, https://doi.org/10.1088/ $1361-6579 / \mathrm{abcdf} 4$

[453] Lai SP, Hsieh CA, Lin YH, Harutaipree T, Lin SC, Peng YH, Cheng LP, Chen MY (2020) StrengthGaming: Enabling Dynamic Repetition Tempo in Strength Training-based Exergame Design. In: 22nd International Conference on Human-Computer Interaction with Mobile Devices and Services, Association for Computing Machinery, New York, NY, USA, MobileHCI '20, pp 1-8, https://doi.org/10.1145/ 3379503.3403529

[454] Lambert H (2006) Accounting for EBM: Notions of evidence in medicine. Social Science \& Medicine 62(11):2633-2645, https://doi.org/10.1016/ j.socscimed.2005.11.023

[455] Landi I, Glicksberg BS, Lee HC, Cherng S, Landi
G, Danieletto M, Dudley JT, Furlanello C, Miotto $R$ (2020) Deep representation learning of electronic health records to unlock patient stratification at scale. npj Digital Medicine 3(1):1-11, https://doi.org/ $10.1038 / \mathrm{s} 41746-020-0301-\mathrm{z}$

[456] Langner T, Gustafsson FK, Avelin B, Strand R, Ahlström H, Kullberg J (2021) Uncertainty-Aware Body Composition Analysis with Deep Regression Ensembles on UK Biobank MRI. arXiv:210106963 [cs, eess] http://arxiv.org/abs/2101.06963

[457] Larsen S, Kristiansen E, Tillaar Rvd (2021) Effects of subjective and objective autoregulation methods for intensity and volume on enhancing maximal strength during resistance-training interventions: a systematic review. PeerJ 9:e10663, http://doi.org/ $10.7717 /$ peerj.10663

[458] Lasevicius T, Ugrinowitsch C, Schoenfeld BJ, Roschel $\mathrm{H}$, Tavares LD, Souza EOD, Laurentino G, Tricoli V (2018) Effects of different intensities of resistance training with equated volume load on muscle strength and hypertrophy. European Journal of Sport Science 18(6):772-780, https://doi.org/ 10.1080/17461391.2018.1450898

[459] Lawford BJ, Delany C, Bennell KL, Hinman RS (2019) "I Was Really Pleasantly Surprised": Firsthand Experience and Shifts in Physical Therapist Perceptions of Telephone-Delivered Exercise Therapy for Knee Osteoarthritis-A Qualitative Study. Arthritis Care \& Research 71(4):545-557, https:// doi.org/10.1002/acr.23618

[460] Lebleu J, Gosseye T, Detrembleur C, Mahaudens P, Cartiaux O, Penta M (2020) Lower Limb Kinematics Using Inertial Sensors during Locomotion: Accuracy and Reproducibility of Joint Angle Calculations with Different Sensor-to-Segment Calibrations. Sensors 20(3):715, https://doi.org/10.3390/ s 20030715

[461] Ledesma A, Bidargaddi N, Strobel J, Schrader G, Nieminen H, Korhonen I, Ermes M (2019) Health timeline: an insight-based study of a timeline visualization of clinical data. BMC Medical Informatics and Decision Making 19(1):170, https://doi.org/ 10.1186/s12911-019-0885-x

[462] Lee H, Troschel FM, Tajmir S, Fuchs G, Mario J, Fintelmann FJ, Do S (2017) Pixel-Level Deep Segmentation: Artificial Intelligence Quantifies Muscle on Computed Tomography for Body Morphometric Analysis. Journal of Digital Imaging 30(4):487-498, https://doi.org/10.1007/s10278-017-9988-z

[463] Lee MH, Siewiorek DP, Smailagic A, Bernardino A, Badia SBi (2020) Opportunities of a Machine Learning-based Decision Support System for Stroke Rehabilitation Assessment. arXiv:200212261 [cs] http://arxiv.org/abs/2002.12261

[464] Lee S, Park M, Lee K, Lee J (2019) Scalable muscle-actuated human simulation and control. ACM Transactions on Graphics 38(4):73:1-73:13, https : //doi.org/10.1145/3306346.3322972

[465] Lee S, Cone SM, Kim S (2020) A biomechanical comparison of self-selected and experimentally controlled speeds and grip widths during the bench press exercise. Sports Biomechanics 0(0):1-13, https: //doi.org/10.1080/14763141.2020.1772357

[466] Lee Y, Kim S, Lee J (2010) Data-driven biped control. ACM Transactions on Graphics 29(4):129:1-129:8, https://doi.org/10.1145/1778765.1781155

[467] Lee Y, Kim M, Lee H (2021) The Measurement of Stiffness for Major Muscles with Shear Wave Elastography and Myoton: A Quantitative Analysis Study. Diagnostics 11(3):524, https://doi.org/ 10.3390 /diagnostics 11030524

[468] Lefundes G, Oliveira L (2020) On estimating gaze by self-attention augmented convolutions. 
arXiv:200811055 [cs] http://arxiv.org/abs/ 2008.11055

[469] Leightley D, Yap MH, Coulson J, Barnouin Y, McPhee JS (2015) Benchmarking human motion analysis using kinect one: An open source dataset. In: 2015 Asia-Pacific Signal and Information Processing Association Annual Summit and Conference (APSIPA), pp 1-7, https://doi.org/10.1109/ APS IPA.2015.7415438

[470] Lemire J (2020) Evaluating NBA Talent: 'Everyone Knows These Things Can Be Done Smarter', https://ww.sporttechie.com/evaluating-nbadraft-talent-scouting-technology-data

[471] Lemire J (2021) Fusion Sport, UFC Performance Institute Team Up. https://wWw.sporttechie.com/ fusion-sport-ufc-performance-institute-teamup

[472] Lenetsky S, Harris N (2012) The Mixed Martial Arts Athlete: A Physiological Profile. Strength \& Conditioning Journal 34(1):32-47, http://doi.org/ $10.1519 /$ SSC.0b013e3182389f00

[473] Levy-Fix G, Kuperman GJ, Elhadad N (2019) Machine Learning and Visualization in Clinical Decision Support: Current State and Future Directions. arXiv:190602664 [cs, stat] http://arxiv.org/ abs / 1906.02664

[474] Lewis J, Mc Auliffe S, O'Sullivan K, O'Sullivan P, Whiteley R (2020) Musculoskeletal Physical Therapy After COVID-19: Time for a New "Normal". Journal of Orthopaedic \& Sports Physical Therapy 51(1):5-7, http://doi.org/10.2519/jospt.2021.0102

[475] Lewis P, Korf HW, Kuffer L, Groß JV, Erren TC (2018) Exercise time cues (zeitgebers) for human circadian systems can foster health and improve performance: a systematic review. BMJ Open Sport \& Exercise Medicine 4(1):e000443, http://dx.doi.org/ 10.1136/bmj sem-2018-000443

[476] Li Y, Muñoz J, Mehrabi S, Middleton L, Cao S, Boger J (2020) Multidisciplinary Iterative Design of Exergames (MIDE): A Framework for Supporting the Design, Development, and Evaluation of Exergames for Health. In: Fang X (ed) HCI in Games, Springer International Publishing, Cham, Lecture Notes in Computer Science, pp 128-147, https://doi.org/ 10.1007/978-3-030-50164-8_9

[477] Liao Y, Vakanski A, Xian M, Paul D, Baker R (2020) A review of computational approaches for evaluation of rehabilitation exercises. Computers in Biology and Medicine 119:103687, https://doi.org/ 10.1016/j.compbiomed.2020.103687

[478] Lillie EO, Patay B, Diamant J, Issell B, Topol EJ, Schork NJ (2011) The n-of-1 clinical trial: the ultimate strategy for individualizing medicine? Personalized Medicine 8(2):161-173, https://doi.org/ $10.2217 /$ pme.11.7

[479] Lim CG, Tsai CY, Chen MY (2020) MuscleSense: Exploring Weight Sensing using Wearable Surface Electromyography (sEMG). In: Proceedings of the Fourteenth International Conference on Tangible, Embedded, and Embodied Interaction, Association for Computing Machinery, New York, NY, USA, TEI '20, pp 255-263, https://doi.org/10.1145/3374920.3374943

[480] Lim ST, Kim DY, Kwon HT, Lee E (2021) Sleep quality and athletic performance according to chronotype. BMC Sports Science, Medicine and Rehabilitation 13(1):2, https://doi.org/10.1186/s13102-020$00228-2$

[481] Lindberg $\mathrm{K}$, Solberg $\mathrm{P}$, Bjørnsen $\mathrm{T}$, Helland C, Rønnestad B, Frank MT, Haugen T, Østerås S, Kristoffersen M, Midttun M, Sæland F, Paulsen G (2021) Force-velocity profiling in athletes: Reliability and agreement across methods. PLOS ONE 16(2):e0245791, https://doi.org/10.1371/journal.pone.0245791

[482] Lindsay R, Lenetsky S (2020) The Contribution of Expert Coaches' Experiential Knowledge in Understanding Punching Performance in Boxers. Journal of Emerging Sport Studies 3(1), https:// digitalcommons.library.tru.ca/jess/vol3/iss $1 / 2$

[483] Lindsell CJ, Stead WW, Johnson KB (2020) ActionInformed Artificial Intelligence-Matching the Algorithm to the Problem. JAMA 323(21):2141-2142, https://doi.org/10.1001/jama.2020.5035

[484] Linnamo V, Pakarinen A, Komi PV, Kraemer WJ, Häkkinen K (2005) Acute hormonal responses to submaximal and maximal heavy resistance and explosive exercises in men and women. Journal of Strength and Conditioning Research 19(3):566-571, https:// pubmed.ncbi.nlm.nih.gov/16095404/

[485] Liu J, Liu X, Zhang Q (2020) A new training method for leg explosive power in taekwondo and its data-driven predictive models. Isokinetics and Exercise Science 28(4):351-363, http://doi.org/10.3233/ IES- 202110

[486] Liu J, Shahroudy A, Perez M, Wang G, Duan LY, Kot AC (2020) NTU RGB+D 120: A Large-Scale Benchmark for 3D Human Activity Understanding. IEEE Transactions on Pattern Analysis and Machine Intelligence 42(10):2684-2701, https://doi.org/10.1109/ TPAMI.2019.2916873

[487] Liu L, Long D, Magerko B (2020) MoViz: A Visualization Tool for Comparing Motion Capture Data Clustering Algorithms. In: Proceedings of the 7th International Conference on Movement and Computing, Association for Computing Machinery, New York, NY, USA, MOCO '20, pp 1-8, https://doi.org/10.1145/ 3401956.3404228

[488] Liu LY, Sangani S, Patterson KK, Fung J, Lamontagne A (2020) Real-Time Avatar-Based Feedback to Enhance the Symmetry of Spatiotemporal Parameters After Stroke: Instantaneous Effects of Different Avatar Views. IEEE Transactions on Neural Systems and Rehabilitation Engineering 28(4):878887, https://doi.org/10.1109/TNSRE.2020.2979830

[489] Liu M, Yang D, Zhang Y, Cui Z, Rehg JM, Tang S (2020) 4D Human Body Capture from Egocentric Video via 3D Scene Grounding. arXiv:201113341 [csCV] https : //arxiv.org/abs/2011.13341v1

[490] Liu P, He G, Zhao L (2021) From Model-driven to Data-driven: A Survey on Active Deep Learning. arXiv:210109933 [cs] http://arxiv.org/abs/ 2101.09933

[491] Liu S, Ostadabbas S (2018) A Semi-Supervised Data Augmentation Approach using 3D Graphical Engines. In: ECCV 2018, pp 0-0, https://arxiv.org/ abs/1808.02595

[492] Liu Z, Zhang H, Chen Z, Wang Z, Ouyang W (2020) Disentangling and Unifying Graph Convolutions for Skeleton-Based Action Recognition. In: Proceedings of the IEEE/CVF Conference on Computer Vision and Pattern Recognition, pp 143-152, https: //openaccess.thecvf.com/content_CVPR_2020/html/ Liu_Disentangling_and_Unifying_Graph_Convolutions_for_Skeleton Based_Action_Recognition_CVPR_2020_paper.html

[493] Lo Presti L, La Cascia M (2016) 3D skeletonbased human action classification: A survey. Pattern Recognition 53:130-147, https://doi.org/10.1016/ j.patcog.2015.11.019

[494] Lohr C, Schmidt T, Medina-Porqueres I, Braumann KM, Reer R, Porthun J (2019) Diagnostic accuracy, validity, and reliability of Tensiomyography to assess muscle function and exercise-induced fatigue in healthy participants. A systematic review with meta-analysis. Journal of Electromyography and Kinesiology 47:65-87, https://doi.org/10.1016/ j.jelekin.2019.05.005 
[495] Lok R, Zerbini G, Gordijn MCM, Beersma DGM, Hut RA (2020) Gold, silver or bronze: circadian variation strongly affects performance in Olympic athletes. Scientific Reports 10(1):16088, https:// doi.org/10.1038/s41598-020-72573-8

[496] Longo UG, Risi Ambrogioni L, Berton A, Candela V, Carnevale A, Schena E, Gugliemelli E, Denaro V (2020) Physical therapy and precision rehabilitation in shoulder rotator cuff disease. International Orthopaedics 44(5):893-903, https://doi.org/10.1007/ s00264-020-04511-2

[497] Longoni L, Brunati R, Sale P, Casale R, Ronconi G, Ferriero G (2019) Smartphone applications validated for joint angle measurement: a systematic review. International Journal of Rehabilitation Research 42(1):11-19, http://doi.org/10.1097/ MRR. 0000000000000332

[498] López-Laval I, Sitko S, Muñiz-Pardos B, Cirer-Sastre R, Calleja-González J (2020) Relationship Between Bench Press Strength and Punch Performance in Male Professional Boxers. The Journal of Strength \& Conditioning Research 34(2):308-312, http:// doi.org/10.1519/JSC.0000000000003362

[499] Lorenzoni V, Staley J, Marchant T, Onderdijk KE, Maes PJ, Leman M (2019) The sonic instructor: A music-based biofeedback system for improving weightlifting technique. PLOS ONE 14(8):e0220915, https://doi.org/10.1371/journal.pone.0220915

[500] Loturco I, Contreras B, Kobal R, Fernandes V, Moura $\mathrm{N}$, Siqueira F, Winckler C, Suchomel T, Pereira LA (2018) Vertically and horizontally directed muscle power exercises: Relationships with top-level sprint performance. PLOS ONE 13(7):e0201475, https:// doi.org/10.1371/journal.pone.0201475

[501] Lu M, Shahn Z, Sow D, Doshi-Velez F, Lehman LwH (2020) Is Deep Reinforcement Learning Ready for Practical Applications in Healthcare? A Sensitivity Analysis of Duel-DDQN for Sepsis Treatment. arXiv:200504301 [cs, stat] http://arxiv.org/ abs/2005.04301

[502] Luo Z, Hachiuma R, Yuan Y, Iwase S, Kitani KM (2020) Kinematics-Guided Reinforcement Learning for Object-Aware 3D Ego-Pose Estimation. arXiv:201104837 [csCV] p 9, https://arxiv.org/ $\mathrm{abs} / 2011.04837$

[503] Lusch B, Kutz JN, Brunton SL (2018) Deep learning for universal linear embeddings of nonlinear dynamics. Nature Communications 9(1):4950, https: //www.nature.com/articles/s41467-018-07210-0

[504] Luu BC, Wright AL, Haeberle HS, Karnuta JM, Schickendantz MS, Makhni EC, Nwachukwu BU, Williams RJ, Ramkumar PN (2020) Machine Learning Outperforms Logistic Regression Analysis to Predict Next-Season NHL Player Injury: An Analysis of 2322 Players From 2007 to 2017. Orthopaedic Journal of Sports Medicine 8(9):2325967120953404, https://doi.org/10.1177/2325967120953404

[505] Machek SB, Lorenz KA, Kern M, Galpin AJ, Bagley JR (2019) Skeletal Muscle Fiber Type and Morphology in a Middle-Aged Elite Male Powerlifter Using Anabolic Steroids. Journal of Science in Sport and Exercise https://doi.org/10.1007/s42978-01900039-z

[506] Maddox WJ, Benton G, Wilson AG (2020) Rethinking Parameter Counting in Deep Models: Effective Dimensionality Revisited. arXiv:200302139 [cs, stat] http://arxiv.org/abs/2003.02139

[507] Maestroni L, Read P, Bishop C, Papadopoulos K, Suchomel TJ, Comfort P, Turner A (2020) The Benefits of Strength Training on Musculoskeletal System Health: Practical Applications for Interdisciplinary Care. Sports Medicine 50(8):1431-1450, https: //doi.org/10.1007/s40279-020-01309-5
[508] Mahyari A, Pirolli P (2021) Physical Exercise Recommendation and Success Prediction Using Interconnected Recurrent Neural Networks. arXiv:201000482 [cs, math, stat] http://arxiv.org/abs/2010.00482

[509] Mai-Nguyen AV, Tran VL, Dao M, Zettsu K (2020) Leverage the Predictive Power Score of Lifelog Data's Attributes to Predict the Expected Athlete Performance. Tech. rep., Ho Chi Minh City University of Science, http://doi.org/10.13140/RG.2.2.32867.58403

[510] Maijala A, Kinnunen H, Koskimäki H, Jämsä T, Kangas M (2019) Nocturnal finger skin temperature in menstrual cycle tracking: ambulatory pilot study using a wearable Oura ring. BMC Women's Health 19(1):150, https://doi.org/10.1186/s12905019-0844-9

[511] Marasingha-Arachchige SU, Rubio-Arias JA, Alcaraz PE, Chung LH (2020) Factors that affect heart rate variability following acute resistance exercise: A systematic review and meta-analysis. Journal of Sport and Health Science https://doi.org/10.1016/ j.jshs.2020.11.008

[512] Marcelino P (2018) Transfer learning from pretrained models. https://towardsdatascience.com/ transfer-learning-from-pre-trained-modelsf2393f 124751

[513] Marco G, Alberto B, Taian V (2017) Surface EMG and muscle fatigue: multi-channel approaches to the study of myoelectric manifestations of muscle fatigue. Physiological Measurement 38(5):R27-R60, https: // doi.org/10.1088/1361-6579/aa60b9

[514] Mareš J (2018) Resistance of health personnel to changes in healthcare. Kontakt 20(3):e262-e272, https://doi.org/10.1016/j.kontakt.2018.04.002

[515] Markofski MM, Braun WA (2014) Influence of Menstrual Cycle on Indices of Contraction-Induced Muscle Damage. The Journal of Strength \& Conditioning Research 28(9):2649-2656, http://doi.org/ $10.1519 /$ JSC. 0000000000000429

[516] Marquez DC, Tscharner Vv, Murari K, Nigg BM (2018) Development of a multichannel currentEMG system for coherence modulation with visual biofeedback. PLOS ONE 13(11):e0206871, https:// doi.org/10.1371/journal.pone.0206871

[517] Marshall JD, Aldarondo DE, Dunn TW, Wang WL, Berman GJ, Ölveczky BP (2021) Continuous WholeBody 3D Kinematic Recordings across the Rodent Behavioral Repertoire. Neuron 109(3):420-437.e8, https://doi.org/10.1016/j.neuron.2020.11.016

[518] Martinez C, Tavakoli M (2020) Learning and Reproduction of Therapist's Semi-Periodic Motions during Robotic Rehabilitation. Robotica 38(2):337-349, https://doi.org/10.1017/S0263574719000651

[519] Martínez-Cava A, Hernández-Belmonte A, CourelIbáñez J, Morán-Navarro R, González-Badillo JJ, Pallarés JG (2020) Reliability of technologies to measure the barbell velocity: Implications for monitoring resistance training. PLOS ONE 15(6):e0232465, https://doi.org/10.1371/journal.pone.0232465

[520] Martínez-Gómez R, Valenzuela PL, Alejo LB, GilCabrera J, Montalvo-Pérez A, Talavera E, Lucia A, Moral-González S, Barranco-Gil D (2020) Physiological Predictors of Competition Performance in CrossFit Athletes. International Journal of Environmental Research and Public Health 17(10):3699, https://doi.org/10.3390/ijerph17103699

[521] Martinez-Nicolas A, Ortiz-Tudela E, Rol MA, Madrid JA (2013) Uncovering Different Masking Factors on Wrist Skin Temperature Rhythm in Free-Living Subjects. PLOS ONE 8(4):e61142, https://doi.org/ 10.1371/journal.pone.0061142

[522] Martorelli AS, Lima FDD, Vieira A, Tufano JJ, Ernesto C, Boullosa D, Bottaro M (2021) The interplay between internal and external load 
parameters during different strength training sessions in resistance-trained men. European Journal of Sport Science 21(1):16-25, https://doi.org/ 10.1080/17461391.2020.1725646

[523] Masood MA, Doshi-Velez F (2019) DiversityInducing Policy Gradient: Using Maximum Mean Discrepancy to Find a Set of Diverse Policies. arXiv:190600088 [cs, stat] http://arxiv.org/abs/ 1906.00088

[524] Massetti T, da Silva TD, Crocetta TB, Guarnieri R, de Freitas BL, Bianchi Lopes P, Watson S, Tonks J, de Mello Monteiro CB (2018) The Clinical Utility of Virtual Reality in Neurorehabilitation: A Systematic Review. Journal of Central Nervous System Disease 10:1179573518813541, https://doi.org/ $10.1177 / 1179573518813541$

[525] Mateen BA, Liley J, Denniston AK, Holmes CC, Vollmer SJ (2020) Improving the quality of machine learning in health applications and clinical research. Nature Machine Intelligence 2(10):554-556, https: //doi.org/10.1038/s42256-020-00239-1

[526] Mateus RB, Ferrer-Roca V, João F, Veloso AP (2020) Muscle contributions to maximal single-leg forward braking and backward acceleration in elite athletes. Journal of Biomechanics 112:110047, https: //doi.org/10.1016/j.jbiomech.2020.110047

[527] Mathis A, Schneider S, Lauer J, Mathis MW (2020) A Primer on Motion Capture with Deep Learning: Principles, Pitfalls, and Perspectives. Neuron 108(1):4465, https://doi.org/10.1016/j.neuron.2020.09.017

[528] Matthews A (2021) Blurring boundaries between humans and technology: postdigital, postphenomenology and actor-network theory in qualitative research. Qualitative Research in Sport, Exercise and Health 13(1):26-40, https://doi.org/10.1080/ 2159676x.2020.1836508

[529] McCormack S, Jones B, Scantlebury S, Rotheram D, Till K (2020) "It's Important, but It's Not Everything": Practitioners' Use, Analysis and Perceptions of Fitness Testing in Academy Rugby League. Sports 8(9):130, https://doi.org/10.3390/ sports 8090130

[530] McCunn R, aus der Fünten K, Fullagar HHK, McKeown I, Meyer T (2016) Reliability and Association with Injury of Movement Screens: A Critical Review. Sports Medicine 46(6):763-781, https: //doi.org/10.1007/s40279-015-0453-1

[531] McGuigan H, Hassmén P, Rosic N, Stevens CJ (2020) Training monitoring methods used in the field by coaches and practitioners: A systematic review. International Journal of Sports Science \& Coaching 15(3):439-451, https://doi.org/10.1177/ 1747954120913172

[532] McGuigan MR, Wright GA, Fleck SJ (2012) Strength Training for Athletes: Does It Really Help Sports Performance? International Journal of Sports Physiology and Performance 7(1):2-5, https:// doi.org/10.1123/ijspp.7.1.2

[533] McLachlan S (2020) Health Information Standardisation as a basis for Learning Health Systems. arXiv:200404811 [cs, math] http://arxiv.org/abs/ 2004.04811

[534] McLoughlin E, Fletcher D, Slavich GM, Arnold R, Moore LJ (2021) Cumulative lifetime stress exposure, depression, anxiety, and well-being in elite athletes: A mixed-method study. Psychology of Sport and Exercise 52:101823, https://doi.org/10.1016/ j.psychsport. 2020.101823

[535] McManus CJ, Collison J, Cooper CE (2018) Performance comparison of the MOXY and PortaMon near-infrared spectroscopy muscle oximeters at rest and during exercise. Journal of Biomedical Optics 23(1):015007, https://doi.org/10.1117/
1.JB0.23.1.015007

[536] Mears A (2019) Investigating the effectiveness of digital technology for elite athlete development and support from athlete, coach and support team perspectives: Final report for the IOC Olympic Studies Centre Advanced Olympic Research Grant Programme 2018/2019 Award. report, Loughborough University

[537] Meeusen R, Duclos M, Foster C, Fry A, Gleeson M, Nieman D, Raglin J, Rietjens G, Steinacker J, Urhausen A, European College of Sport Science, American College of Sports Medicine (2013) Prevention, diagnosis, and treatment of the overtraining syndrome: joint consensus statement of the European College of Sport Science and the American College of Sports Medicine. Medicine and Science in Sports and Exercise 45(1):186-205, https: //doi.org/ $10.1249 / \mathrm{mss} .0 \mathrm{~b} 013 \mathrm{e} 318279 \mathrm{a} 10 \mathrm{a}$

[538] Mehl K, Strobel M (2020) Monitoring the performance of physical exercises. https:// patents.google.com/patent/US20200410220A1/en?q= G06K9/00342\&sort=new

[539] Mehta SP (2019) Clinical relevance commentary in response to "The validity and reliability of DrGoniometer, a smartphone application, for measuring forearm supination". Journal of Hand Therapy 32(1):118-120, https://doi.org/10.1016/ j.jht.2018.12.001

[540] Mejia-Trujillo JD, Castaño-Pino YJ, Navarro A, Arango-Paredes JD, Rincón D, Valderrama J, Muñoz B, Orozco JL (2019) Kinect ${ }^{\mathrm{TM}}$ and Intel RealSense ${ }^{\mathrm{TM}} \mathrm{D} 435$ comparison: a preliminary study for motion analysis. In: 2019 IEEE International Conference on E-health Networking, Application \& Services (HealthCom), IEEE, pp 1-4, https://doi.org/ 10.1109/HealthCom46333.2019.9009433

[541] Menaspà MJ, Menaspà P, Clark SA, Fanchini M (2018) Validity of the Online Athlete Management System to Assess Training Load. International Journal of Sports Physiology and Performance 13(6):750-754, https://doi.org/10.1123/ ij spp. 2017-0379

[542] Mendt S, Gunga HC, Felsenberg D, Belavy DL, Steinach M, Stahn AC (2021) Regular exercise counteracts circadian shifts in core body temperature during long-duration bed rest. npj Microgravity 7(1):1-6, https://doi.org/10.1038/s41526-020-00129-1

[543] Mengarelli A, Verdini F, Cardarelli S, Di Nardo F, Burattini L, Fioretti S (2018) Balance assessment during squatting exercise: A comparison between laboratory grade force plate and a commercial, lowcost device. Journal of Biomechanics 71:264-270, https://doi.org/10.1016/j.jbiomech.2018.01.029

[544] Mesin L, Merlo E, Merletti R, Orizio C (2010) Investigation of motor unit recruitment during stimulated contractions of tibialis anterior muscle. Journal of Electromyography and Kinesiology 20(4):580-589, https://doi.org/10.1016/j.jelekin.2009.11.008

[545] Meskó B, Görög M (2020) A short guide for medical professionals in the era of artificial intelligence. npj Digital Medicine 3(1):1-8, https://doi.org/10.1038/ s41746-020-00333-z

[546] Michalopoulos N, Katsini C, Raptis GE (2019) Using Video-based Technology in Powerlifting Sport to Support Referees' Decision Making. In: Extended Abstracts of the 2019 CHI Conference on Human Factors in Computing Systems, Association for Computing Machinery, New York, NY, USA, CHI EA '19, pp 1-6, https://doi.org/10.1145/3290607.3312991

[547] Mikalef P, Gupta M (2021) Artificial Intelligence Capability: Conceptualization, measurement calibration, and empirical study on its impact on organizational creativity and firm performance. Information 
\& Management p 103434, https://doi.org/10.1016/ j.im.2021.103434

[548] Miles A (2017) From evidence-based to evidenceinformed, from patient-focussed to personcentered-The ongoing "energetics" of health and social care discourse as we approach the Third Era of Medicine. Journal of Evaluation in Clinical Practice 23(1):3-4, https://doi.org/10.1111/jep.12733

[549] Militaru C, Militaru MD, Benta KI (2020) Physical Exercise Form Correction Using Neural Networks. In: Companion Publication of the 2020 International Conference on Multimodal Interaction, Association for Computing Machinery, New York, NY, USA, ICMI '20 Companion, pp 240-244, https://doi.org/ $10.1145 / 3395035.3425302$

[550] Mind OT (2020) Will Your Job Exist in the Future? https://podtail.com/podcast/one-trackmind-1/02-will-your-job-exist-in-the-future/

[551] Miotto R, Danieletto M, Scelza JR, Kidd BA, Dudley JT (2018) Reflecting health: smart mirrors for personalized medicine. npj Digital Medicine 1(1):17, https://doi.org/10.1038/s41746-018-0068-7

[552] Mirmomeni M, Fazio T, von Cavallar S, Harrer S (2021) From wearables to THINKables: artificial intelligence-enabled sensors for health monitoring. In: Sazonov E (ed) Wearable Sensors (Second Edition), Academic Press, Oxford, pp 339-356, https: //doi.org/10.1016/B978-0-12-819246-7.00012-7

[553] Moen E, Bannon D, Kudo T, Graf W, Covert M, Van Valen D (2019) Deep learning for cellular image analysis. Nature Methods 16(12):1233-1246, https: //doi.org/10.1038/s41592-019-0403-1

[554] Mohr AE, Jäger R, Carpenter KC, Kerksick CM, Purpura M, Townsend JR, West NP, Black K, Gleeson M, Pyne DB (2020) The athletic gut microbiota. Journal of the International Society of Sports Nutrition 17:133, https://dx.doi.org/10.1186/s12970-020-00353W

[555] Monti E, Toniolo L, Marcucci L, Bondì M, Martellato I, Šimunič B, Toninello P, Franchi MV, Narici MV, Reggiani C (2020) Are muscle fibres of body builders intrinsically weaker? A comparison with single fibres of aged-matched controls. Acta Physiologica n/a(n/a):e13557, https:// doi.org/10.1111/apha.13557

[556] Montoya M, Henao O, Muñoz J (2017) Muscle fatigue detection through wearable sensors: a comparative study using the myo armband. In: Proceedings of the XVIII International Conference on Human Computer Interaction, Association for Computing Machinery, New York, NY, USA, Interacci\&\#xf3;n '17, pp 1-2, https://doi.org/10.1145/3123818.3123855

[557] Moore J, Dorrell H (2020) Guidelines and Resources for Prescribing Load using Velocity Based Training. International Universities Strength and Conditioning Association Journal 1(1), http://doi.org/10.47206/ iuscaj.v1i1.4

[558] Moore JB (2020) From personalised nutrition to precision medicine: the rise of consumer genomics and digital health. Proceedings of the Nutrition Society 79(3):300-310, http://doi.org/10.1017/ S0029665120006977

[559] Moreira DG, Costello JT, Brito CJ, Adamczyk JG, Ammer K, Bach AJE, Costa CMA, Eglin C, Fernandes AA, Fernández-Cuevas I, Ferreira JJA, Formenti D, Fournet D, Havenith G, Howell K, Jung A, Kenny GP, Kolosovas-Machuca ES, Maley MJ, Merla A, Pascoe DD, Priego Quesada JI, Schwartz RG, Seixas ARD, Selfe J, Vainer BG, SilleroQuintana M (2017) Thermographic imaging in sports and exercise medicine: A Delphi study and consensus statement on the measurement of human skin temperature. Journal of Thermal Biology 69:155-162, https://doi.org/10.1016/j.jtherbio.2017.07.006

[560] Morgenstern JD, Rosella LC, Daley MJ, Goel V, Schünemann HJ, Piggott T (2021) "AI's gonna have an impact on everything in society, so it has to have an impact on public health": a fundamental qualitative descriptive study of the implications of artificial intelligence for public health. BMC Public Health 21(1):40, https://doi.org/10.1186/s12889$020-10030-x$

[561] Morton J, Witherden FD, Kochenderfer MJ (2019) Deep Variational Koopman Models: Inferring Koopman Observations for Uncertainty-Aware Dynamics Modeling and Control. arXiv:190209742 [cs, stat] http: //arxiv.org/abs/1902.09742

[562] Morton RW, Colenso-Semple L, Phillips SM (2019) Training for strength and hypertrophy: an evidence-based approach. Current Opinion in Physiology 10:90-95, https://doi.org/10.1016/j.cophys.2019.04.006

[563] Morton RW, Colenso-Semple L, Phillips SM (2019) Training for strength and hypertrophy: an evidence-based approach. Current Opinion in Physiology 10:90-95, https://doi.org/10.1016/j.cophys.2019.04.006

[564] Morville T, Sahl RE, Moritz T, Helge JW, Clemmensen C (2020) Plasma Metabolome Profiling of Resistance Exercise and Endurance Exercise in Humans. Cell Reports 33(13), https://doi.org/ 10.1016/j.celrep.2020.108554

[565] Mueller CM, Dweck CS (1998) Praise for intelligence can undermine children's motivation and performance. Journal of Personality and Social Psychology $75(1): 33-52$, https://doi.org/10.1037//00223514.75.1.33

[566] Müller AR, Brands MM, Ven PMvd, Roes KC, Cornel MC, Karnebeek CDv, Wijburg FA, Daams JG, Boot E, Eeghen AMv (2021) The Power of 1: Systematic Review of N-of-1 Studies in Rare Genetic Neurodevelopmental Disorders. Neurology https:// doi.org/10.1212/WNL.0000000000011597

[567] Muñoz JE, Cao S, Boger J (2019) Kinematically Adaptive Exergames: Personalizing Exercise Therapy Through Closed-Loop Systems. In: 2019 IEEE International Conference on Artificial Intelligence and Virtual Reality (AIVR), pp 118-1187, https://doi.org/ 10.1109/A IVR46125.2019.00026

[568] Muoio D (2020) HHS' final interoperability rules standardize APIs for patient health data access through apps. https://www.mobihealthnews.com/ news/hhs - final - inter operability-rules -

standardize-apis-patient-health-data-accessthrough-apps

[569] Murphy P, Claxton L, Hodgson R, Glynn D, Beresford L, Walton M, Llewellyn A, Palmer S, Dias S (2021) Exploring Heterogeneity in Histology-Independent Technologies and the Implications for Cost-Effectiveness. Medical Decision Making 41(2):165-178, https://doi.org/10.1177/ 0272989x20980327

[570] Mustafa B, Loh A, Freyberg J, MacWilliams P, Wilson M, McKinney SM, Sieniek M, Winkens J, Liu Y, Bui P, Prabhakara S, Telang U, Karthikesalingam A, Houlsby N, Natarajan V (2021) Supervised Transfer Learning at Scale for Medical Imaging. arXiv:210105913 [cs] http://arxiv.org/ abs/2101.05913

[571] Mustalampi S, Häkkinen A, Kautiainen H, Weir A, Ylinen J (2013) Responsiveness of Muscle Tone Characteristics to Progressive Force Production. The Journal of Strength \& Conditioning Research 27(1):159-165, http://doi.org/10.1519/ JSC.0b013e3182518023

[572] Myllyaho MM, Ihalainen JK, Hackney AC, Valtonen 
M, Nummela A, Vaara E, Häkkinen K, Kyröläinen H, Taipale RS (2021) Hormonal Contraceptive Use Does Not Affect Strength, Endurance, or Body Composition Adaptations to Combined Strength and Endurance Training in Women. The Journal of Strength \& Conditioning Research 35(2):449-457, http:// doi.org/10.1519/JSC.0000000000002713

[573] Naeemabadi M, Dinesen B, Andersen OK, Hansen J (2019) Influence of a Marker-Based Motion Capture System on the Performance of Microsoft Kinect v2 Skeleton Algorithm. IEEE Sensors Journal 19(1):171179

[574] Nag N, Pandey V, Jain RC (2018) Endogenous and Exogenous Multi-Modal Layers in Context Aware Recommendation Systems for Health. arXiv: 180806468 [csCY] https://arxiv.org/abs/1808.06468v1

[575] Nakano N, Sakura T, Ueda K, Omura L, Kimura A, Iino Y, Fukashiro S, Yoshioka S (2020) Evaluation of 3D Markerless Motion Capture Accuracy Using OpenPose With Multiple Video Cameras. Frontiers in Sports and Active Living 2, https://dx.doi.org/ 10.3389 /fspor 2020.00050

[576] Nanayakkara T, Clermont G, Langmead CJ, Swigon D (2021) Unifying Cardiovascular Modelling with Deep Reinforcement Learning for Uncertainty Aware Control of Sepsis Treatment. arXiv:210108477 [cs, qbiol http://arxiv.org/abs/2101.08477

[577] Nasri N, Orts-Escolano S, Cazorla M (2020) An sEMG-Controlled 3D Game for Rehabilitation Therapies: Real-Time Time Hand Gesture Recognition Using Deep Learning Techniques. Sensors 20(22):6451, https://doi.org/10.3390/s20226451

[578] Network STR (2020) AR / VR / MR in Sports - Where's research in relation to industry? https://anchor.fm/strp/episodes/Chad-Goebert- - AR - - VR- - MR - in-Sports - - -Wheres - research- inrelation-to-industry-ej93mn

[579] Newbold J, Gold NE, Bianchi-Berthouze N (2020) Movement sonification expectancy model: leveraging musical expectancy theory to create movementaltering sonifications. Journal on Multimodal User Interfaces 14(2):153-166, https://doi.org/10.1007/ s12193-020-00322-2

[580] Ng AY (2020) Practical limitations of today's deep learning in healthcare. https: //ml4health.github.io/

[581] Ni J, Muhlstein L, McAuley J (2019) Modeling Heart Rate and Activity Data for Personalized Fitness Recommendation. In: The World Wide Web Conference, Association for Computing Machinery, New York, NY, USA, WWW '19, pp 1343-1353, https: //doi.org/10.1145/3308558.3313643

[582] Nieman DC, Pence BD (2020) Exercise immunology: Future directions. Journal of Sport and Health Science 9(5):432-445, https://doi.org/ 10.1016/j.jshs.2019.12.003

[583] Nijem RM, Coburn JW, Brown LE, Lynn SK, Ciccone AB (2016) Electromyographic and Force Plate Analysis of the Deadlift Performed With and Without Chains. The Journal of Strength \& Conditioning Research 30(5):1177-1182, http://doi.org/10.1519/ JSC. 0000000000001351

[584] Nishiwaki GA, Urabe Y, Tanaka K (2006) EMG Analysis of Lower Extremity Muscles in Three Different Squat Exercises. Journal of the Japanese Physical Therapy Association 9(1):21-26, https://doi.org/ 10.1298/jjpta.9.21

[585] Norgeot B, Glicksberg BS, Butte AJ (2019) A call for deep-learning healthcare. Nature Medicine 25(1):1415, https://doi.org/10.1038/s41591-018-0320-3

[586] Nusinovici S, Tham YC, Chak Yan MY, Wei Ting DS, Li J, Sabanayagam C, Wong TY, Cheng CY (2020) Logistic regression was as good as machine learn- ing for predicting major chronic diseases. Journal of Clinical Epidemiology 122:56-69, https://doi.org/ $10.1016 / j . j c l i n e p i .2020 .03 .002$

[587] Ochoa GG (2013) Development of a wearable freeweight exercise assistant. Master's thesis, Technical University of Eindhoven

[588] O'Connor D, Larkin P, Robertson S, Goodyear P (2021) The art of the question: the structure of questions posed by youth soccer coaches during training. Physical Education and Sport Pedagogy 0(0):1-16, https://doi.org/10.1080/17408989.2021.1877270

[589] Ogata R, Simo-Serra E, Iizuka S, Ishikawa H (2019) Temporal Distance Matrices for Squat Classification. In: Proceedings of the IEEE/CVF Conference on Computer Vision and Pattern Recognition Workshops, pp 0-0, https://doi.org/10.1109/ CVPRW.2019.00309

[590] Oh TH, Jaroensri R, Kim C, Elgharib M, Durand F, Freeman WT, Matusik W (2018) Learningbased Video Motion Magnification. arXiv preprint arXiv: 180402684

[591] Oliveira RMD, Ugrinowitsch C, Kingsley JD, Silva DGD, Bittencourt D, Caruso FR, Borghi-Silva A, Libardi CA (2019) Effect of individualized resistance training prescription with heart rate variability on individual muscle hypertrophy and strength responses. European Journal of Sport Science 19(8):1092-1100, https://doi.org/10.1080/17461391.2019.1572227

[592] Olivera ALP, Alzapiedi DF, Solan MC, Karamanidis K, Mileva KN, James DC (2020) Direct muscle electrical stimulation as a method for the in vivo assessment of force production in $\mathrm{m}$. abductor hallucis. Journal of Biomechanics 100:109606, https: //doi.org/10.1016/j.jbiomech.2020.109606

[593] Onnela JP (2021) Opportunities and challenges in the collection and analysis of digital phenotyping data. Neuropsychopharmacology 46(1):45-54, https: //doi.org/10.1038/s41386-020-0771-3

[594] Orange ST, Metcalfe JW, Marshall P, Vince RV, Madden LA, Liefeith A (2020) Test-Retest Reliability of a Commercial Linear Position Transducer (GymAware PowerTool) to Measure Velocity and Power in the Back Squat and Bench Press. The Journal of Strength \& Conditioning Research 34(3):728-737, http://doi.org/10.1519/ JSC. 0000000000002715

[595] Osgouei RH, Soulsby D, Bello F (2020) Rehabilitation Exergames: Use of Motion Sensing and Machine Learning to Quantify Exercise Performance in Healthy Volunteers. JMIR Rehabilitation and Assistive Technologies 7(2):e17289, https://doi.org/ $10.2196 / 17289$

[596] Osório C AM (2019) Performance Evaluation: Subjectivity, Bias and Judgement Style in Sport Data. SSRN https://dx.doi.org/10.2139/ssrn.3321838

[597] Owusu G (2007) AI and computer-based methods in performance evaluation of sporting feats: an overview. Artificial Intelligence Review 27(1):57-70, https://doi.org/10.1007/s10462-008-9068-3

[598] Paay J, Kjeldskov J, Sorensen F, Jensen T, Tirosh O (2019) Weight-Mate: Adaptive Training Support for Weight Lifting. In: Proceedings of the 31st Australian Conference on Human-Computer-Interaction, Association for Computing Machinery, New York, NY, USA, OZCHI'19, pp 95-105, https://doi.org/ 10.1145/3369457.3369466

[599] Pacey R (2020) Pacey Performance Podcast: \#168 Sam Robertson (Head of Research \& Innovation at Western Bulldogs and Victoria University) on Apple Podcasts. https://podcasts.apple.com/gb/podcast/ 168-sam-robertson-head-research-innovationat-western/id799023260?i=1000398569223

[600] Padilla J, Leary E, Limberg JK (2020) Identifying 
responders versus non-responders: Incorporation of controls is required for sound statistical inference. Experimental Physiology n/a(n/a), https://doi.org/ 10.1113/EP089142

[601] Padua DA, Oñate JA (2020) Training Load, Recovery, and Injury: A Simple or Complex Relationship? Journal of Athletic Training 55(9):873-873, https://doi.org/10.4085/1062-6050-055.09

[602] Pan M, Salvi S, Brady E (2018) Designing Auditory Feedback from Wearable Weightlifting Devices. In: Extended Abstracts of the 2018 CHI Conference on Human Factors in Computing Systems, Association for Computing Machinery, New York, NY, USA, CHI EA '18, pp 1-6, https://doi.org/10.1145/ 3170427.3188692

[603] Panayotov V (2020) Relationships between body dimensions and strength abilities in experienced Olympic weightlifters, powerlifters and bodybuilders. Series on Biomechanics http://jsb.imbm.bas.bg/ page/en/details.php?article_id=432

[604] Paoli A, Mancin L, Saoncella M, Grigoletto D, Pacelli FQ, Zamparo P, Schoenfeld BJ, Marcolin G (2019) Mind-muscle connection: effects of verbal instructions on muscle activity during bench press exercise. European Journal of Translational Myology 29(2), https://dx.doi.org/10.4081/ejtm.2019.8250

[605] Pareja-Blanco F, Alcazar J, Cornejo-Daza PJ Sánchez-Valdepeñas J, Rodriguez-Lopez C, Mora JHd, Sánchez-Moreno M, Bachero-Mena B, Alegre LM, Ortega-Becerra M (2020) Effects of velocity loss in the bench press exercise on strength gains, neuromuscular adaptations, and muscle hypertrophy. Scandinavian Journal of Medicine \& Science in Sports 30(11):2154-2166, http://doi.org/10.1249/ MSS.0000000000002295

[606] Parisi GI, Stosch Fv, Magg S, Wermter S (2015) Learning human motion feedback with neural selforganization. In: 2015 International Joint Conference on Neural Networks (IJCNN), pp 1-6, https: //doi.org/10.1109/IJCNn.2015.7280701

[607] Parisi GI, Magg S, Wermter S (2016) Human motion assessment in real time using recurrent selforganization. In: 2016 25th IEEE International Symposium on Robot and Human Interactive Communication (RO-MAN), pp 71-76, https://doi.org/ 10.1109/ROMAN.2016.7745093

[608] Parisi GI, Kemker R, Part JL, Kanan C, Wermter S (2019) Continual lifelong learning with neural networks: A review. Neural Networks 113:54-71, https: //doi.org/10.1016/j.neunet.2019.01.012

[609] Park H, Wang ZJ, Das N, Paul AS, Perumalla P, Zhou Z, Chau DH (2021) SkeletonVis: Interactive Visualization for Understanding Adversarial Attacks on Human Action Recognition Models. arXiv:210110586 [cs] http://arxiv.org/abs/2101.10586

[610] Park HJ, Baek JW, Kim JH (2020) Imagery based Parametric Classification of Correct and Incorrect Motion for Push-up Counter Using OpenPose. In: 2020 IEEE 16th International Conference on Automation Science and Engineering (CASE), pp 13891394

[611] Park HS, Lee GA, Seo BK, Billinghurst M (2020) User experience design for a smart-mirror-based personalized training system. Multimedia Tools and Applications https://doi.org/10.1007/s11042-02010148-5

[612] Park J, Kim SJ, Na Y, Kim Y, Kim J (2019) Development of a Bendable Outsole Biaxial Ground Reaction Force Measurement System. Sensors 19(11):2641, https://doi.org/10.3390/s19112641

[613] Park Sm, Won DD, Lee BJ, Escobedo D, Esteva A, Aalipour A, Ge TJ, Kim JH, Suh S, Choi EH, Lozano AX, Yao C, Bodapati S, Achterberg FB, Kim J,
Park H, Choi Y, Kim WJ, Yu JH, Bhatt AM, Lee JK, Spitler R, Wang SX, Gambhir SS (2020) A mountable toilet system for personalized health monitoring via the analysis of excreta. Nature Biomedical Engineering 4(6):624-635, https://doi.org/10.1038/s41551$020-0534-9$

[614] Parr EB, Heilbronn LK, Hawley JA (2020) A Time to Eat and a Time to Exercise. Exercise and Sport Sciences Reviews 48(1):4-10, https://dx.doi.org/ 10.1249/JES.0000000000000207

[615] Patel BN, Rosenberg L, Willcox G, Baltaxe D, Lyons M, Irvin J, Rajpurkar P, Amrhein T, Gupta R, Halabi S, Langlotz C, Lo E, Mammarappallil J, Mariano AJ, Riley G, Seekins J, Shen L, Zucker E, Lungren MP (2019) Human-machine partnership with artificial intelligence for chest radiograph diagnosis. npj Digital Medicine 2(1):1-10, https://doi.org/10.1038/ s41746-019-0189-7

[616] Patel D, Shah D, Shah M (2020) The Intertwine of Brain and Body: A Quantitative Analysis on How Big Data Influences the System of Sports. Annals of Data Science 7(1):1-16, https: //doi.org/10.1007/ s40745-019-00239-y

[617] Patil AK, Balasubramanyam A, Ryu JY, B N PK, Chakravarthi B, Chai YH (2020) Fusion of Multiple Lidars and Inertial Sensors for the Real-Time Pose Tracking of Human Motion. Sensors 20(18):5342, https://doi.org/10.3390/s20185342

[618] Peake JM, Neubauer O, Della Gatta PA, Nosaka K (2016) Muscle damage and inflammation during recovery from exercise. Journal of Applied Physiology 122(3):559-570, https://doi.org/ 10.1152/japplphysio1.00971.2016

[619] Pellatt L, Dewar A, Philippides A, Roggen D (2021) Mapping Vicon Motion Tracking to 6-Axis IMU Data for Wearable Activity Recognition. In: Ahad MAR, Inoue S, Roggen D, Fujinami K (eds) Activity and Behavior Computing, Smart Innovation, Systems and Technologies, Springer, Singapore, pp 3-20, https: //doi.org/10.1007/978-981-15-8944-7_1

[620] Pellicer-Valero OJ, Cattinelli I, Neri L, Mari F, Martín-Guerrero JD, Barbieri C (2020) Enhanced prediction of hemoglobin concentration in a very large cohort of hemodialysis patients by means of deep recurrent neural networks. Artificial Intelligence in Medicine 107:101898, https://doi.org/10.1016/ j.artmed.2020.101898

[621] Perakslis E, Ginsburg GS (2021) Digital HealthThe Need to Assess Benefits, Risks, and Value. JAMA 325(2):127-128, https://doi.org/10.1001/ jama.2020.22919

[622] Pereira HM, Larson RD, Bemben DA (2020) Menstrual Cycle Effects on Exercise-Induced Fatigability. Frontiers in Physiology 11, https://dx.doi.org/ 10.3389/fphys.2020.00517

[623] Pérez-Castilla A, Piepoli A, Garrido-Blanca G, Delgado-García G, Balsalobre-Fernández C, GarcíaRamos A (2019) Precision of 7 Commercially Available Devices for Predicting Bench-Press 1-Repetition Maximum From the Individual Load-Velocity Relationship. International Journal of Sports Physiology and Performance 14(10):1442-1446, https://doi.org/10.1123/ijspp.2018-0801

[624] Perrey S (2020) Exercise: A Gate That Primes the Brain to Perform. Brain Sciences 10(12):980, https: //doi.org/10.3390/brainsci10120980

[625] Petersen BK, Yang J, Grathwohl WS, Cockrell C, Santiago C, An G, Faissol DM (2018) Precision medicine as a control problem: Using simulation and deep reinforcement learning to discover adaptive, personalized multi-cytokine therapy for sepsis. arXiv:180210440 [cs, q-bio] http://arxiv.org/abs/ 1802.10440 
[626] Pfister A, West AM, Bronner S, Noah JA (2014) Comparative abilities of Microsoft Kinect and Vicon 3D motion capture for gait analysis. Journal of Medical Engineering \& Technology 38(5):274-280, https : //doi.org/10.3109/03091902.2014.909540

[627] Pichardo AW, Oliver JL, Harrison CB, Maulder PS, Lloyd RS, Kandoi R (2019) Effects of Combined Resistance Training and Weightlifting on Injury Risk Factors and Resistance Training Skill of Adolescent Males. Journal of Strength and Conditioning Research https://doi.org/10.1519/ jsc.0000000000003341

[628] Pickering C (2020) The Role of Compression Garments in Performance and Recovery. https://simplifaster.com/articles/compressiongarments-performance-recovery/

[629] Pickering C, Kiely J (2019) The Development of a Personalised Training Framework: Implementation of Emerging Technologies for Performance. Journal of Functional Morphology and Kinesiology 4(2):25, https://doi.org/10.3390/jfmk4020025

[630] Pizzolato C, Shim VB, Lloyd DG, Devaprakash D, Obst SJ, Newsham-West R, Graham DF, Besier TF, Zheng MH, Barrett RS (2020) Targeted Achilles Tendon Training and Rehabilitation Using Personalized and Real-Time Multiscale Models of the Neuromusculoskeletal System. Frontiers in Bioengineering and Biotechnology 8, https://doi.org/10.3389/ fbioe.2020.00878

[631] Plews DJ, Scott B, Altini M, Wood M, Kilding AE, Laursen PB (2017) Comparison of HeartRate-Variability Recording With Smartphone Photoplethysmography, Polar H7 Chest Strap, and Electrocardiography. International Journal of Sports Physiology and Performance 12(10):1324-1328, https://doi.org/10.1123/ijspp.2016-0668

[632] Plizzari C, Cannici M, Matteucci M (2020) Skeletonbased Action Recognition via Spatial and Temporal Transformer Networks. arXiv:200807404 [cs] http:// arxiv.org/abs/2008.07404

[633] Poltronieri TS, Paula NSd, Chaves GV (2020) Assessing skeletal muscle radiodensity by computed tomography: An integrative review of the applied methodologies. Clinical Physiology and Functional Imaging 40(4):207-223, https://doi.org/10.1111/cpf.12629

[634] Potter T, Vieira R, de Roos B (2021) Perspective: Application of N-of-1 Methods in Personalized Nutrition Research. Advances in Nutrition (nmaa173), https://doi.org/10.1093/advances/nmaa173

[635] Poudineh M, Maikawa CL, Ma EY, Pan J, Mamerow D, Hang Y, Baker SW, Beirami A, Yoshikawa A, Eisenstein M, Kim S, Vučković J, Appel EA, Soh HT (2020) A fluorescence sandwich immunoassay for the real-time continuous detection of glucose and insulin in live animals. Nature Biomedical Engineering pp 111, https://doi.org/10.1038/s41551-020-00661-1

[636] Poulos CCN (2019) Exploring the Relationship between Performance on a Series of Squat based Movement Tasks and Passive Range of Motion Capacity - Is Successful Performance a Valid Method of Ruling out Range of Motion Restrictions? Thesis, University of Toronto, https:// tspace.library.utoronto.ca/handle/1807/98285

[637] Powers R, Etezadi-Amoli M, Arnold EM, Kianian S, Mance I, Gibiansky M, Trietsch D, Alvarado AS, Kretlow JD, Herrington TM, Brillman S, Huang N, Lin PT, Pham HA, Ullal AV (2021) Smartwatch inertial sensors continuously monitor real-world motor fluctuations in Parkinson's disease. Science Translational Medicine 13(579), http://doi.org/10.1126/ scitranslmed.abd7865

[638] Powles J, Hodson H (2017) Google DeepMind and healthcare in an age of algorithms. Health and
Technology 7(4):351-367, https://doi.org/10.1007/ s 12553-017-0179-1

[639] Prabhu G, O'Connor NE, Moran K (2020) Recognition and Repetition Counting for Local Muscular Endurance Exercises in Exercise-Based Rehabilitation: A Comparative Study Using Artificial Intelligence Models. Sensors 20(17):4791, https://doi.org/ $10.3390 / \mathrm{s} 20174791$

[640] Prasad N, Engelhardt BE, Doshi-Velez F (2019) Defining Admissible Rewards for High Confidence Policy Evaluation. arXiv:190513167 [cs, stat] http: //arxiv.org/abs/1905.13167

[641] Priego-Quesada JI, De la Fuente C, Kunzler MR, Perez-Soriano P, Hervás-Marín D, Carpes FP (2020) Relationship between Skin Temperature, Electrical Manifestations of Muscle Fatigue, and ExerciseInduced Delayed Onset Muscle Soreness for Dynamic Contractions: A Preliminary Study. International Journal of Environmental Research and Public Health 17(18):6817, https://doi.org/10.3390/ ijerph17186817

[642] Prosperi M, Min JS, Bian J, Modave F (2018) Big data hurdles in precision medicine and precision public health. BMC Medical Informatics and Decision Making 18(1):139, https://doi.org/10.1186/ s 12911-018-0719-2

[643] Pueo B, Penichet-Tomas A, Jimenez-Olmedo JM (2020) Validity, reliability and usefulness of smartphone and kinovea motion analysis software for direct measurement of vertical jump height. Physiology \& Behavior 227:113144, https://doi.org/10.1016/ j.physbeh.2020.113144

[644] Pullinger SA, Oksa J, Brocklehurst EL, Iveson RP, Newlove A, Burniston JG, Doran DA, Waterhouse JM, Edwards BJ (2018) Controlling rectal and muscle temperatures: Can we offset diurnal variation in repeated sprint performance? Chronobiology International 35(7):959-968, https://doi.org/10.1080/ 07420528.2018 .1444626

[645] Qin H, Zhang S, Liu Q, Chen L, Chen B (2020) PointSkelCNN: Deep Learning-Based 3D Human Skeleton Extraction from Point Clouds. Computer Graphics Forum 39(7):363-374, https://doi.org/10.1111/ $\operatorname{cgf} .14151$

[646] Qin Y, Pan S, Zhou W, Pan D, Li Z (2020) Consumer Wi-Fi device based action quality recognition: An illustrative example of seated dumbbell press action. IET Communications $n / a(n / a)$, https: //doi.org/10.1049/cmu2.12093

[647] Quinn KM, Cox AJ, Roberts LA, Briskey D, Minahan C (2020) Reliability of a point-of-care device to determine oxidative stress in whole blood before and after acute exercise: A practical approach for the applied sports sciences. Journal of Sports Sciences 0(0):1-10, https://doi.org/10.1080/ 02640414.2020 .1840755

[648] Rabbi F, Park T, Fang B, Zhang M, Lee Y (2018) When Virtual Reality Meets Internet of Things in the Gym: Enabling Immersive Interactive Machine Exercises. Proceedings of the ACM on Interactive, Mobile, Wearable and Ubiquitous Technologies 2(2):78:178:21, https://doi.org/10.1145/3214281

[649] Racinais S, Oksa J (2010) Temperature and neuromuscular function. Scandinavian Journal of Medicine \& Science in Sports 20(s3):1-18, https:// doi.org/10.1111/j.1600-0838.2010.01204.x

[650] Radcliffe K, Lyson HC, Barr-Walker J, Sarkar U (2019) Collective intelligence in medical decisionmaking: a systematic scoping review. BMC Medical Informatics and Decision Making 19(1):158, https: //doi.org/10.1186/s12911-019-0882-0

[651] Radhakrishnan M, Misra A, Balan RK (2020) W8-Scope: Fine-grained, practical monitoring of 
weight stack-based exercises. In: Proceedings of the 18th Annual IEEE International Conference on Persuasive Computing and Communications (PerCom2020), https://doi.org/10.1109/ PerCom45495.2020.9127379

[652] Rajbhandary PL, Nallathambi G (2020) Feasibility of Continuous Monitoring of Core Body Temperature Using Chest-worn Patch Sensor. In: 2020 42nd Annual International Conference of the IEEE Engineering in Medicine Biology Society (EMBC), pp 4652-4655, https://doi.org/10.1109/ EMBC44109.2020.9175579

[653] Rajšp A, Fister I (2020) A Systematic Literature Review of Intelligent Data Analysis Methods for Smart Sport Training. Applied Sciences 10(9):3013, https : //doi.org/10.3390/app10093013

[654] Ramalingam S (2019) Comparison of Resistance Training Progression Models to Develop Muscular Endurance in Youth Athletes: Applications for Athlete Monitoring. Thesis, Auckland University of Technology, https://openrepository.aut.ac.nz/handle/ 10292/12865

[655] Ramos-Campo D, Martínez-Aranda LM, Caravaca LA, Ávila-Gandí V, Rubio-Arias JÁ (2020) Effects of resistance training intensity on the sleep quality and strength recovery in trained men: a randomized cross-over study. Biology of Sport 38(1):81-88, https://doi.org/10.5114/biolsport.2020.97677

[656] Rana M, Mittal V (2021) Wearable Sensors for Real-Time Kinematics Analysis in Sports: A Review. IEEE Sensors Journal 21(2):1187-1207, https : //doi.org/10.1109/JSEN.2020.3019016

[657] Rane L, Ding Z, McGregor AH, Bull AMJ (2019) Deep Learning for Musculoskeletal Force Prediction. Annals of Biomedical Engineering 47(3):778789, https://doi.org/10.1007/s10439-018-02190-0

[658] Ransbotham S, Khoadandeh S, Kiron D, Candelon F, Chu M, LaFountain B (2020) Expanding AI's Impact With Organizational Learning. MIT Sloan Management Review and Boston Consulting Group https: //sloanreview.mit.edu/projects/expanding-ais impact-with-organizational-learning/

[659] Raykar VC, Yu S, Zhao LH, Jerebko A, Florin C, Valadez GH, Bogoni L, Moy L (2009) Supervised learning from multiple experts: whom to trust when everyone lies a bit. In: Proceedings of the 26th Annual International Conference on Machine Learning, Association for Computing Machinery, Montreal, Quebec, Canada, ICML '09, pp 889-896, https://doi.org/ 10.1145/1553374.1553488

[660] Rea DJ, Schneider S, Kanda T (2021) "Is this all you can do? Harder!": The Effects of (Im)Polite Robot Encouragement on Exercise Effort. In: 2021 $\mathrm{ACM} / \mathrm{IEEE}$ International Conference on HumanRobot Interaction, Boulder, CO, USA

[661] Rector K, Bennett CL, Kientz JA (2013) Eyes-free yoga: an exergame using depth cameras for blind \&amp; low vision exercise. In: Proceedings of the 15th International ACM SIGACCESS Conference on Computers and Accessibility, Association for Computing Machinery, New York, NY, USA, ASSETS '13, pp 1-8, https://doi.org/10.1145/2513383.2513392

[662] Reece MB, Arnold GP, Nasir S, Wang WW, Abboud R (2020) Barbell back squat: how do resistance bands affect muscle activation and knee kinematics? BMJ Open Sport \& Exercise Medicine 6(1):e000610, http: //dx.doi.org/10.1136/bmj sem-2019-000610

[663] Reenalda J, Maartens E, Buurke JH, Gruber AH (2019) Kinematics and shock attenuation during a prolonged run on the athletic track as measured with inertial magnetic measurement units. Gait \& Posture 68:155-160, https://doi.org/10.1016/ j.gaitpost.2018.11.020
[664] Reggiani C, Schiaffino S (2020) Muscle hypertrophy and muscle strength: dependent or independent variables? A provocative review. European Journal of Translational Myology 30(3), https://dx.doi.org/ 10.4081/ejtm.2020.9311

[665] Reiss A, Stricker D (2012) Creating and benchmarking a new dataset for physical activity monitoring. In: Proceedings of the 5th International Conference on PErvasive Technologies Related to Assistive Environments, Association for Computing Machinery, New York, NY, USA, PETRA '12, pp 1-8, https: //doi.org/10.1145/2413097.2413148

[666] Remedios SM, Armstrong DP, Graham RB, Fischer SL (2020) Exploring the Application of Pattern Recognition and Machine Learning for Identifying Movement Phenotypes During Deep Squat and Hurdle Step Movements. Frontiers in Bioengineering and Biotechnology 8, https://doi.org/10.3389/ fbioe. 2020.00364

[667] Renaissance Periodization (2020) Dr. James Hoffmann on Athlete Testing and Monitoring. https: //youtu.be/c7b_gh6Th18

[668] Renner E, Lang N, Langenstein B, Struck M, Bertsch $\mathrm{T}$ (2020) Validating sweat ammonia as physiological parameter for wearable devices in sports science*. In: 2020 42nd Annual International Conference of the IEEE Engineering in Medicine Biology Society (EMBC), pp 4644-4647, https://doi.org/10.1109/ EMBC44109.2020.9175434

[669] Rensburg DCJv, Rensburg AJv, Fowler P, Fullagar $\mathrm{H}$, Stevens D, Halson S, Bender A, Vincent G, Claassen-Smithers A, Dunican I, Roach GD, Sargent C, Lastella M, Cronje T (2020) How to manage travel fatigue and jet lag in athletes? A systematic review of interventions. British Journal of Sports Medicine 54(16):960-968, http://dx.doi.org/ 10.1136/bj sports-2019-101635

[670] Reya M, Škarabot J, Cveticanin B, Šarabon N (2020) Factors Underlying Bench Press Performance in Elite Competitive Powerlifters. The Journal of Strength \& Conditioning Research Publish Ahead of Print, http: //doi.org/10.1519/JSC.0000000000003097

[671] Reynolds JM, Gordon TJ, Robergs RA (2006) Prediction of one repetition maximum strength from multiple repetition maximum testing and anthropometry. Journal of Strength and Conditioning Research 20(3):584-592, https://doi.org/10.1519/r15304.1

[672] Rhoden EL, Morgentaler A (2004) Risks of Testosterone-Replacement Therapy and Recommendations for Monitoring. New England Journal of Medicine 350(5):482-492, https://doi.org/10.1056/ NEJMra022251

[673] Ribera d'Alcala E (2020) Machine learning approaches for state prediction and motion simulation in a human musculoskeletal model based on inertial motion tracking data. PhD thesis, Politecnico Milano, https://www.politesi.polimi.it/ handle/10589/165081

[674] Richter C, King E, Strike S, Franklyn-Miller A (2018) Analyzing Human Movements - Introducing A Framework To Extract And Evaluate Biomechanical Data. bioRxiv p 442087, https://doi.org/10.1101/ 442087

[675] Richter C, King E, Strike S, Franklyn-Miller A (2019) Objective classification and scoring of movement deficiencies in patients with anterior cruciate ligament reconstruction. PLOS ONE 14(7):e0206024, https: //doi.org/10.1371/journal.pone.0206024

[676] Rieke N, Hancox J, Li W, Milletarì F, Roth HR, Albarqouni S, Bakas S, Galtier MN, Landman BA, Maier-Hein K, Ourselin S, Sheller M, Summers RM, Trask A, Xu D, Baust M, Cardoso MJ (2020) The 
future of digital health with federated learning. npj Digital Medicine 3(1):1-7, https://doi.org/10.1038/ s41746-020-00323-1

[677] Rigamonti L, Albrecht UV, Lutter C, Tempel M, Wolfarth B, Back DA, Digitalisation WG (2020) Potentials of Digitalization in Sports Medicine: A Narrative Review. Current Sports Medicine Reports 19(4):157163, http://doi.org/10.1249/JSR.0000000000000704

[678] Rigamonti L, Estel K, Gehlen T, Wolfarth B, Lawrence JB, Back DA (2021) Use of artificial intelligence in sports medicine: a report of 5 fictional cases. BMC Sports Science, Medicine and Rehabilitation 13(1):13, https://doi.org/10.1186/s13102$021-00243-x$

[679] Rittweger J, Moss AD, Colier W, Stewart C, Degens H (2010) Muscle tissue oxygenation and VEGF in VO2-matched vibration and squatting exercise. Clinical Physiology and Functional Imaging 30(4):269-278, https://doi.org/10.1111/ j.1475-097X.2010.00937.x

[680] Robert-Lachaine X, Mecheri H, Muller A, Larue C, Plamondon A (2020) Validation of a low-cost inertial motion capture system for whole-body motion analysis. Journal of Biomechanics 99:109520, https: //doi.org/10.1016/j.jbiomech.2019.109520

[681] Roberts BM, Lavin KM, Many GM, ThalackerMercer A, Merritt EK, Bickel CS, Mayhew DL, Tuggle SC, Cross JM, Kosek DJ, Petrella JK, Brown CJ, Hunter GR, Windham ST, Allman RM, Bamman MM (2018) Human neuromuscular aging: Sex differences revealed at the myocellular level. Experimental Gerontology 106:116-124, https://doi.org/10.1016/ j.exger.2018.02.023

[682] Roberts BM, Nuckols G, Krieger JW (2020) Sex Differences in Resistance Training: A Systematic Review and Meta-Analysis. The Journal of Strength \& Conditioning Research 34(5):1448-1460, http://doi.org/ $10.1519 / \mathrm{JSC} .0000000000003521$

[683] Robertson CM, Pullinger SA, Robinson WR, Smith ME, Burniston JG, Waterhouse JM, Edwards BJ (2018) Is the diurnal variation in muscle force output detected/detectable when multijoint movements are analysed using the musclelab force-velocity encoder? Chronobiology International 35(10):1391-1401, https://doi.org/10.1080/ 07420528.2018 .1485685

[684] Robertson PS (2020) Man \& machine: Adaptive tools for the contemporary performance analyst. Journal of Sports Sciences 38(18):2118-2126, https://doi.org/ 10.1080/02640414.2020.1774143

[685] Rodrigues P, Trajano GS, Wharton L, Minett GM (2020) Effects of passive heating intervention on muscle hypertrophy and neuromuscular function: A preliminary systematic review with meta-analysis. Journal of Thermal Biology 93:102684, https:// doi.org/10.1016/j.jtherbio.2020.102684

[686] Rodríguez MÁ, García-Calleja P, Terrados N, Crespo I, Valle Md, Olmedillas H (2020) Injury in CrossFit $\AA$ : A Systematic Review of Epidemiology and Risk Factors. The Physician and Sportsmedicine 0(ja):null, https://doi.org/10.1080/00913847.2020.1864675

[687] Romero-Moraleda B, Coso JD, Gutiérrez-Hellín J, Ruiz-Moreno C, Grgic J, Lara B (2019) The Influence of the Menstrual Cycle on Muscle Strength and Power Performance. Journal of Human Kinetics 68(1):123133, https://doi.org/10.2478/hukin-2019-0061

[688] Ross GB, Dowling B, Troje NF, Fischer SL, Graham RB (2018) Objectively Differentiating Movement Patterns between Elite and Novice Athletes. Medicine and Science in Sports and Exercise 50(7):1457-1464, https://doi.org/10.1249/mss.00000000000001571

[689] Ross MK, Broz F, Baillie L (2020) Informing the Design of a Robotic Coach through Systematic Obser- vations. In: Companion of the $2020 \mathrm{ACM} / \mathrm{IEEE}$ International Conference on Human-Robot Interaction, Association for Computing Machinery, New York, NY, USA, HRI '20, pp 412-414, https://doi.org/ $10.1145 / 3371382.3378351$

[690] Ross R, Goodpaster BH, Koch LG, Sarzynski MA, Kohrt WM, Johannsen NM, Skinner JS, Castro A, Irving BA, Noland RC, Sparks LM, Spielmann G, Day AG, Pitsch W, Hopkins WG, Bouchard C (2019) Precision exercise medicine: understanding exercise response variability. British Journal of Sports Medicine 53(18):1141-1153, http://dx.doi.org/10.1136/ bjsports-2018-100328

[691] Rubio-Sastre P, Gómez-Abellán P, Martinez-Nicolas A, Ordovás JM, Madrid JA, Garaulet M (2014) Evening physical activity alters wrist temperature circadian rhythmicity. Chronobiology International 31(2):276-282, https://doi.org/10.3109/ 07420528.2013 .833215

[692] Ruiz-Muñoz M, Cuesta-Vargas AI (2014) Electromyography and sonomyography analysis of the tibialis anterior: a cross sectional study. Journal of Foot and Ankle Research 7(1):11, https://doi.org/ 10.1186/1757-1146-7-11

[693] Ryu H, Kim M, Lee S, Park MS, Lee K, Lee J (2020) Functionality-Driven Musculature Retargeting. arXiv:200715311 [cs] http://arxiv.org/abs/ 2007.15311

[694] Sá ECd, Medeiros AR, Ferreira AS, Ramos AG, Janicijevic D, Boullosa D (2019) Validity of the iLOAD® app for resistance training monitoring. PeerJ 7:e7372, http://doi.org/10.7717/peerj.7372

[695] Saeed A, Ozcelebi T, Lukkien J (2019) Multi-task Self-Supervised Learning for Human Activity Detection. Proceedings of the ACM on Interactive, Mobile, Wearable and Ubiquitous Technologies 3(2):61:161:30, https://doi.org/10.1145/3328932

[696] Sammut S (2020) Organizational Antecedents to the Implementation of Precision Medicine: Overcoming Resistance to Change. Executive Doctorate in Business Administration, Temple University, Philadelphia, Pennsylvania, http://dx.doi.org/ $10.34944 /$ dspace $/ 280$

[697] Sánchez-Medina L, González-Badillo JJ (2011) Velocity Loss as an Indicator of Neuromuscular Fatigue during Resistance Training. Medicine \& Science in Sports \& Exercise 43(9):1725-1734, http: //doi.org/10.1249/MSS.0b013e318213f880

[698] Sánchez-Medina L, González-Badillo JJ, Pérez CE, Pallarés JG (2014) Velocity- and power-load relationships of the bench pull vs. bench press exercises. International Journal of Sports Medicine 35(3):209-216, https://doi.org/10.1055/s-0033-1351252

[699] Sands WA, Kavanaugh AA, Murray SR, McNeal JR, Jemni M (2017) Modern Techniques and Technologies Applied to Training and Performance Monitoring. International Journal of Sports Physiology and Performance 12(Suppl 2):S263-S272, https://doi.org/ 10.1123/ijspp.2016-0405

[700] Sanford JA, Nogiec CD, Lindholm ME, Adkins JN, Amar D, Dasari JK Surendra anhulsmd Drugan, Fernández FM, Radom-Aizik S, Schenk S, Snyder MP, Tracy RP, Vanderboom P, Trappe S, Walsh MJ, Adkins JN, Amar D, Dasari S, Drugan JK, Evans CR, Fernandez FM, Li Y, Lindholm ME, Nogiec CD, Radom-Aizik S, Sanford JA, Schenk S, Snyder MP, Tomlinson L, Tracy RP, Trappe S, Vanderboom P, Walsh MJ, Lee Alekel D, Bekirov I, Boyce AT, Boyington J, Fleg JL, Joseph LJO, Laughlin MR, Maruvada P, Morris SA, McGowan JA, Nierras C, Pai V, Peterson C, Ramos E, Roary MC, Williams JP, Xia A, Cornell E, Rooney J, Miller ME, Ambrosius WT, Rushing S, Stowe CL, Jack Rejeski W, Nicklas 
BJ, Pahor M, Lu Cj, Trappe T, Chambers T, Raue U, Lester B, Bergman BC, Bessesen DH, Jankowski CM, Kohrt WM, Melanson EL, Moreau KL, Schauer IE, Schwartz RS, Kraus WE, Slentz CA, Huffman KM, Johnson JL, Willis LH, Kelly L, Houmard JA, Dubis G, Broskey N, Goodpaster BH, Sparks LM, Coen PM, Cooper DM, Haddad F, Rankinen T, Ravussin E, Johannsen N, Harris M, Jakicic JM, Newman AB, Forman DD, Kershaw E, Rogers RJ, Nindl BC, Page LC, Stefanovic-Racic M, Barr SL, Rasmussen BB, Moro T, Paddon-Jones D, Volpi E, Spratt H, Musi N, Espinoza S, Patel D, Serra M, Gelfond J, Burns A, Bamman MM, Buford TW, Cutter GR, Bodine SC, Esser K, Farrar RP, Goodyear LJ, Hirshman MF, Albertson BG, Qian WJ, Piehowski P, Gritsenko MA, Monore ME, Petyuk VA, McDermott JE, Hansen JN, Hutchison C, Moore S, Gaul DA, Clish CB, AvilaPacheco J, Dennis C, Kellis M, Carr S, Jean-Beltran PM, Keshishian H, Mani DR, Clauser K, Krug K, Mundorff C, Pearce C, Ivanova AA, Ortlund EA, Maner-Smith K, Uppal K, Zhang T, Sealfon SC, Zaslavsky E, Nair V, Li S, Jain N, Ge Y, Sun Y, Nudelman G, Ruf-zamojski F, Smith G, Pincas N, Rubenstein A, Anne Amper M, Seenarine N, Lappalainen T, Lanza IR, Sreekumaran Nair K, Klaus K, Montgomery SB, Smith KS, Gay NR, Zhao B, Hung CJ, Zebarjadi N, Balliu B, Fresard L, Burant CF, Li JZ, Kachman M, Soni T, Raskind AB, Gerszten R, Robbins J, Ilkayeva O, Muehlbauer MJ, Newgard CB, Ashley EA, Wheeler MT, Jimenez-Morales D, Raja A, Dalton KP, Zhen J, Suk Kim Y, Christle JW, Marwaha S, Chin ET, Hershman SG, Hastie T, Tibshirani R, Rivas MA (2020) Molecular Transducers of Physical Activity Consortium (MoTrPAC): Mapping the Dynamic Responses to Exercise. Cell 181(7):14641474, https://doi.org/10.1016/j.cell.2020.06.004

[701] Sanni AA, McCully KK (2019) Interpretation of Near-Infrared Spectroscopy (NIRS) Signals in Skeletal Muscle. J Funct Morphol Kinesiol 4(2):28, https://doi.org/10.3390/jfmk4020028

[702] Saraceni N, Kent P, Ng L, Campbell A, Straker L, O'Sullivan P (2019) To Flex or Not to Flex? Is There a Relationship Between Lumbar Spine Flexion During Lifting and Low Back Pain? A Systematic Review With Meta-analysis. Journal of Orthopaedic \& Sports Physical Therapy 50(3):121-130, http://doi.org/10.2519/jospt.2020.9218

[703] Sárándi I, Linder T, Arras KO, Leibe B (2018) Synthetic Occlusion Augmentation with Volumetric Heatmaps for the 2018 ECCV PoseTrack Challenge on 3D Human Pose Estimation. arXiv:180904987 [cs] http://arxiv.org/abs/1809.04987

[704] Sardari F, Paiement A, Hannuna S, Mirmehdi M (2020) VI-Net-View-Invariant Quality of Human Movement Assessment. Sensors 20(18):5258, https: //doi.org/10.3390/s20185258

[705] Saripalle R, Runyan C, Russell M (2019) Using HL7 FHIR to achieve interoperability in patient health record. Journal of Biomedical Informatics 94:103188, https://doi.org/10.1016/j.jbi.2019.103188

[706] Sarma KV, Harmon S, Sanford T, Roth HR, Xu Z, Tetreault J, Xu D, Flores MG, Raman AG, Kulkarni R, Wood BJ, Choyke PL, Priester AM, Marks LS, Raman SS, Enzmann D, Turkbey B, Speier W, Arnold CW (2021) Federated learning improves site performance in multicenter deep learning without data sharing. Journal of the American Medical Informatics Association (ocaa341), https://doi.org/10.1093/ jamia/ocaa341

[707] Sartori M, Sawicki G (2021) Closing the loop between wearable technology and human biology: A new paradigm for steering neuromuscular form and function. Progress in Biomedical Engineering https:// doi.org/10.1088/2516-1091/abe3e0

[708] Saw AE, Main LC, Gastin PB (2016) Monitoring the athlete training response: subjective selfreported measures trump commonly used objective measures: a systematic review. British Journal of Sports Medicine 50(5):281-291, http://dx.doi.org/ 10.1136/bj sports-2015-094758

[709] Saxby DJ, Killen BA, Pizzolato C, Carty CP, Diamond LE, Modenese L, Fernandez J, Davico G, Barzan M, Lenton G, da Luz SB, Suwarganda E, Devaprakash D, Korhonen RK, Alderson JA, Besier TF, Barrett RS, Lloyd DG (2020) Machine learning methods to support personalized neuromusculoskeletal modelling. Biomechanics and Modeling in Mechanobiology 19(4):1169-1185, https://doi.org/ 10.1007/s10237-020-01367-8

[710] Scano A, Mira RM, Cerveri P, Molinari Tosatti L, Sacco M (2020) Analysis of Upper-Limb and Trunk Kinematic Variability: Accuracy and Reliability of an RGB-D Sensor. Multimodal Technologies and Interaction 4(2):14, https://doi.org/10.3390/mti4020014

[711] Schaffert N, Janzen TB, Mattes K, Thaut MH (2019) A Review on the Relationship Between Sound and Movement in Sports and Rehabilitation. Frontiers in Psychology 10, https://doi.org/10.1109/ ACCESS. 2020.3003652

[712] van Schaik JE, Dominici N (2020) Chapter 5 - Motion tracking in developmental research: Methods, considerations, and applications. In: Hunnius S, Meyer M (eds) Progress in Brain Research, New Perspectives on Early Social-cognitive Development, vol 254, Elsevier, pp 89-111, https://doi.org/10.1016/ bs.pbr.2020.06.007

[713] Schmidt SL (ed) (2020) 21st Century Sports - How Technologies Will Change Sports in the Digital Age. Springer, http://doi.org/10.1007/978-3-03050801-2

[714] Schmidt SL (2020) How Technologies Impact Sports in the Digital Age. In: Schmidt SL (ed) 21st Century Sports: How Technologies Will Change Sports in the Digital Age, Future of Business and Finance, Springer International Publishing, Cham, pp 3-14, https:// doi.org/10.1007/978-3-030-50801-2_1

[715] Schmidt SL, Stoneham K (2020) Beyond 2030: What Sports Will Look like for the Athletes, Consumers, and Managers. In: Schmidt SL (ed) 21st Century Sports: How Technologies Will Change Sports in the Digital Age, Future of Business and Finance, Springer International Publishing, Cham, pp 293-301, https: //doi.org/10.1007/978-3-030-50801-2_18

[716] Schoenfeld B (2020) Science and Development of Muscle Hypertrophy, second edition edn. Human Kinetics, Inc., Champaign, IL

[717] Schoenfeld BJ, McCarthy JP (2020) Bodybuilding and Physique Special Topic Issue. Strength \& Conditioning Journal 42(5):1, http://doi.org/10.1519/ SSC. 0000000000000586

[718] Schoenfeld BJ, Grgic J, Contreras B, Delcastillo K, Alto A, Haun C, De Souza EO, Vigotsky AD (2020) To Flex or Rest: Does Adding No-Load Isometric Actions to the Inter-Set Rest Period in Resistance Training Enhance Muscular Adaptations? A Randomized-Controlled Trial. Frontiers in Physiology 10, https://doi.org/10.3389/fphys.2019.01571

[719] Schreiber C, Moissenet F (2019) A multimodal dataset of human gait at different walking speeds established on injury-free adult participants. Scientific Data 6(1):111, https://www.nature.com/articles/ s41597-019-0124-4

[720] Schulz MA, Yeo BTT, Vogelstein JT, MouraoMiranada J, Kather JN, Kording K, Richards B, Bzdok D (2020) Different scaling of linear models and deep learning in UKBiobank brain images 
versus machine-learning datasets. Nature Communications 11(1):4238, https://doi.org/10.1038/s41467$020-18037-z$

[721] Schuur F, Rezazade Mehrizi MH, Ranschaert E (2021) Training opportunities of artificial intelligence $(\mathrm{AI})$ in radiology: a systematic review. European Radiology https ://doi.org/10.1007/ s00330-020-07621-y

[722] Schwartz SM, Wildenhaus K, Bucher A, Byrd B (2020) Digital Twins and the Emerging Science of Self: Implications for Digital Health Experience Design and "Small" Data. Frontiers in Computer Science 2, https://doi.org/10.3389/f comp.2020.00031

[723] Science OOD (2020) Continuous Delivery for Machine Learning. https://medium.com/@ODSC/continuous delivery-for-machine- learning-d07f2d0f051

[724] Scott J, Funk C, Ravichandran B, Challis JH, Collins RT, Liu Y (2020) From Kinematics To Dynamics: Estimating Center of Pressure and Base of Support from Video Frames of Human Motion. arXiv:200100657 [csCV] https: //arxiv.org/abs/2001.00657v1

[725] Sculley D, Holt G, Golovin D, Davydov E, Phillips T, Ebner D, Chaudhary V, Young M, Crespo JF, Dennison D (2015) Hidden Technical Debt in Machine Learning Systems. In: Cortes C, Lawrence ND, Lee DD, Sugiyama M, Garnett R (eds) Advances in Neural Information Processing Systems 28, Curran Associates, Inc., pp 2503-2511, https://dl.acm.org/ doi/abs/10.5555/2969442.2969519

[726] Sebernegg A, Kán P, Kaufmann H (2020) Motion Similarity Modeling - A State of the Art Report. arXiv:200805872 [cs] http://arxiv.org/abs/ 2008.05872

[727] Sedliak M, Finni T, Peltonen J, Häkkinen K (2008) Effect of time-of-day-specific strength training on maximum strength and EMG activity of the leg extensors in men. Journal of Sports Sciences 26(10):1005-1014, https://doi.org/10.1080/ 02640410801930150

[728] Seethapathi N, Wang S, Saluja R, Blohm G, Kording KP (2019) Movement science needs different pose tracking algorithms. arXiv:190710226 [cs, q-bio] http://arxiv.org/abs/1907.10226

[729] Seligson ND, Warner JL, Dalton WS, Martin D, Miller RS, Patt D, Kehl KL, Palchuk MB, Alterovitz G, Wiley LK, Huang M, Shen F, Wang Y, Nguyen KA, Wong AF, Meric-Bernstam F, Bernstam EV, Chen JL (2020) Recommendations for patient similarity classes: results of the AMIA 2019 workshop on defining patient similarity. Journal of the American Medical Informatics Association 27(11):18081812, https://doi.org/10.1093/jamia/ocaa159

[730] Sendak MP, D'Arcy J, Kashyap S, Gao M, Nichols M, Corey K, Ratliff W, Balu S (2020) A path for translation of machine learning products into healthcare delivery. EMJ Innov 10:19-00172, https://doi.org/ $10.33590 /$ emj innov/19-00172

[731] Sepas-Moghaddam A, Etemad A (2021) Deep Gait Recognition: A Survey. arXiv:210209546 [cs] http: //arxiv.org/abs/2102.09546

[732] Sestino A, Mauro AD (2021) Leveraging Artificial Intelligence in Business: Implications, Applications and Methods. Technology Analysis \& Strategic Management 0(0):1-14, https://doi.org/10.1080/ 09537325.2021 .1883583

[733] Shahroudy A, Liu J, Ng TT, Wang G (2016) NTU RGB +D: A Large Scale Dataset for 3D Human Activity Analysis. arXiv:160402808 [cs] http: //arxiv.org/ abs / 1604.02808

[734] Shao D, Zhao Y, Dai B, Lin D (2020) FineGym: A Hierarchical Video Dataset for Fine-grained Action Understanding. arXiv:200406704 [cs] http:// arxiv.org/abs/2004.06704
[735] Sheibani S, Capua L, Kamaei S, Akbari SSA, Zhang J, Guerin H, Ionescu AM (2021) Extended gate field-effect-transistor for sensing cortisol stress hormone. Communications Materials 2(1):1-10, https: //doi.org/10.1038/s43246-020-00114-x

[736] Shi L, Zhang Y, Cheng J, Lu H (2019) TwoStream Adaptive Graph Convolutional Networks for Skeleton-Based Action Recognition. In: 2019 IEEE/CVF Conference on Computer Vision and Pattern Recognition (CVPR), pp 12018-12027, https: //doi.org/10.1109/CVPR.2019.01230

[737] Shi Y, Siddharth N, Paige B, Torr PHS (2019) Variational Mixture-of-Experts Autoencoders for MultiModal Deep Generative Models. arXiv:191103393 [cs, stat] http: //arxiv.org/abs/1911.03393

[738] Shih B, Shah D, Li J, Thuruthel TG, Park YL, Iida F, Bao Z, Kramer-Bottiglio R, Tolley MT (2020) Electronic skins and machine learning for intelligent soft robots. Science Robotics 5(41), http://doi.org/ 10.1126/scirobotics.aaz9239

[739] Shimada S, Golyanik V, Xu W, Theobalt C (2020) PhysCap: Physically Plausible Monocular 3D Motion Capture in Real Time. arXiv:200808880 [cs] http:// arxiv.org/abs/2008.08880

[740] Shrestha S, Jain S (2021) A Bayesian-bandit adaptive design for N-of-1 clinical trials. Statistics in Medicine $\mathrm{n} / \mathrm{a}(\mathrm{n} / \mathrm{a})$, https://doi.org/10.1002/sim.8873

[741] Shrestha YR, Krishna V, von Krogh G (2021) Augmenting organizational decision-making with deep learning algorithms: Principles, promises, and challenges. Journal of Business Research 123:588-603, https://doi.org/10.1016/j.jbusres.2020.09.068

[742] Sierotowicz M, Connan M, Castellini C (2020) Human-In-The-Loop Assessment of an Ultralight, Low-Cost Body Posture Tracking Device. Sensors 20(3):890, https://doi.org/10.3390/s20030890

[743] Siewe J, Marx G, Knöll P, Eysel P, Zarghooni K, Graf M, Herren C, Sobottke R, Michael J (2014) Injuries and Overuse Syndromes in Competitive and Elite Bodybuilding. International Journal of Sports Medicine 35(11):943-948, http://doi.org/10.1055/ s-0034- 1367049

[744] Signini ÉF, Nieman DC, Silva CD, Sakaguchi CA, Catai AM (2020) Oxylipin Response to Acute and Chronic Exercise: A Systematic Review. Metabolites 10(6):264, https://doi.org/10.3390/ metabo10060264

[745] Silva AC, Silva A, Edwards BJ, Tod D, Souza Amaral A, de Alcântara Borba D, Grade I, Túlio de Mello M (2021) Sleep extension in athletes: what we know so far - A systematic review. Sleep Medicine 77:128-135, https://doi.org/10.1016/j.sleep.2020.11.028

[746] Silveira ML, Carvalho TL, Neto AF, Filho TB (2019) A Multi-Kinect System for Serious Game Development Using ROS and Unity. In: Costa-Felix R, Machado JC, Alvarenga AV (eds) XXVI Brazilian Congress on Biomedical Engineering, Springer, Singapore, IFMBE Proceedings, pp 585-591, https:// doi.org/10.1007/978-981-13-2119-1_91

[747] Singh A, Le T, Le Nguyen T, Whelan D, O'Reilly M, Caulfield B, Ifrim G (2021) Interpretable Classification of Human Exercise Videos through Pose Estimation and Multivariate Time Series Analysis. In: 5th International Workshop on Health Intelligence at AAAI-21

[748] Sjöberg H, Aasa U, Rosengren M, Berglund L (2020) Content Validity Index and Reliability of a New Protocol for Evaluation of Lifting Technique in the Powerlifting Squat and Deadlift. The Journal of Strength \& Conditioning Research 34(9):2528-2536, http://doi.org/10.1519/JSC.0000000000002791

[749] Skerik T, Chrpa L, Faber W, Vallati M (2018) Automated Training Plan Generation for Athletes. 
In: 2018 IEEE International Conference on Systems, Man, and Cybernetics (SMC), pp 3865-3870, https: //doi.org/10.1109/SMC.2018.00655

[750] Slater GJ, Dieter BP, Marsh DJ, Helms ER, Shaw G, Iraki J (2019) Is an Energy Surplus Required to Maximize Skeletal Muscle Hypertrophy Associated With Resistance Training. Frontiers in Nutrition 6, https://doi.org/10.3389/fnut.2019.00131

[751] Smith SM, Nichols TE (2018) Statistical Challenges in "Big Data" Human Neuroimaging. Neuron 97(2):263-268, https://doi.org/10.1016/ j.neuron. 2017.12.018

[752] Snarr RL, Tolusso DV, Hallmark AV, Esco MR (2021) Validity of Wearable Electromyographical Compression Shorts to Predict Lactate Threshold During Incremental Exercise in Healthy Subjects. The Journal of Strength \& Conditioning Research 35(3):702-708, http://doi.org/10.1519/JSC.0000000000002721

[753] So C (2020) Human-in-the-Loop Design Cycles - A Process Framework that Integrates Design Sprints, Agile Processes, and Machine Learning with $\mathrm{Hu}-$ mans. In: Degen H, Reinerman-Jones L (eds) Artificial Intelligence in HCI, Springer International Publishing, Cham, Lecture Notes in Computer Science, pp 136-145, https://doi.org/10.1007/978-3$030-50334-5 \_9$

[754] Sohal D, Ragsdell G, Hislop D, Brown P (2020) Revisiting the Knowledge Management Audit: Learning from Practice in a High-Performance Sport Organisation. In: European Conference on Knowledge Management, Academic Conferences International Limited, Kidmore End, United Kingdom, pp 747755,XXII, http://doi.org/10.34190/EKM.20.103

[755] Soligard T, Schwellnus M, Alonso JM, Bahr R, Clarsen B, Dijkstra HP, Gabbett $\mathrm{T}$, Gleeson $\mathrm{M}$, Hägglund M, Hutchinson MR, Rensburg CJv, Khan KM, Meeusen R, Orchard JW, Pluim BM, Raftery M, Budgett R, Engebretsen L (2016) How much is too much? (Part 1) International Olympic Committee consensus statement on load in sport and risk of injury. British Journal of Sports Medicine 50(17):1030-1041, http://dx.doi.org/10.1136/ bjsports-2016-096581

[756] Soliński M, Kuklik P, Giera \ltowski J, Baranowski R, Graff B, \Zebrowski J (2020) The effect of persistent U-shaped patterns in RR night-time series on the heart rate variability complexity in healthy humans. Physiological Measurement 41(6):065001, https:// doi.org/10.1088/1361-6579/ab9376

[757] Solórzano S, Espinosa-Alvarez PD, Jimenes-Vargas K, Pérez-Medina JL (2021) Using Serious Games and Motion Tracking for Physical Rehabilitation. In: Karwowski W, Ahram T, Etinger D, Tanković N, Taiar R (eds) Human Systems Engineering and Design III, Springer International Publishing, Cham, Advances in Intelligent Systems and Computing, pp 180-185, https://doi.org/10.1007/978-3-030-58282-1_29

[758] Song S, Kidziński Ł, Peng XB, Ong C, Hicks J, Levine S, Atkeson CG, Delp SL (2020) Deep reinforcement learning for modeling human locomotion control in neuromechanical simulation. bioRxiv p 2020.08.11.246801, https://doi.org/ 10.1101/2020.08.11.246801

[759] Soriano MA, Kipp K, Lake JP, Suchomel TJ, Marín PJ, Baranda MPSD, Comfort P (2020) Mechanical power production assessment during weightlifting exercises. A systematic review. Sports Biomechanics 0(0):1-27, https://doi.org/10.1080/ 14763141.2020.1747529

[760] Spathis D, Perez-Pozuelo I, Brage S, Wareham NJ, Mascolo C (2020) Self-supervised transfer learning of physiological representations from free-living wearable data. arXiv:201112121 [cs, eess] http:// arxiv.org/abs/2011.12121

[761] Sperger J, Freeman NLB, Jiang X, Bang D, Marchi Dd, Kosorok MR (2020) The future of precision health is data-driven decision support. Statistical Analysis and Data Mining: The ASA Data Science Journal 13(6):537-543, https://doi.org/ $10.1002 /$ sam. 11475

[762] Spijkerman R, Hesselink L, Bertinetto C, Bongers CC, Hietbrink F, Vrisekoop N, Leenen LP, Hopman MT, Jansen JJ, Koenderman L (2020) Analysis of human neutrophil phenotypes as biomarker to monitor exercise-induced immune changes. Journal of Leukocyte Biology n/a(n/a), https://doi.org/10.1002/ JLB. 5A $0820-436 R$

[763] Spitz RW, Bell ZW, Wong V, Yamada Y, Song JS, Buckner SL, Abe T, Loenneke JP (2020) Strength testing or strength training: considerations for future research. Physiological Measurement 41(9):09TR01, https://doi.org/10.1088/1361-6579/abb1fa

[764] Squarcini CFR, Pires MLN, Lopes C, BeneditoSilva AA, Esteves AM, Cornelissen-Guillaume G, Matarazzo C, Garcia D, da Silva MSP, Tufik S, de Mello MT (2013) Free-running circadian rhythms of muscle strength, reaction time, and body temperature in totally blind people. European Journal of Applied Physiology 113(1):157-165, https://doi.org/ $10.1007 / \mathrm{s} 00421-012-2415-8$

[765] Stanley E (2020) Maximal punching performance in amateur boxing: An examination of biomechanical and physical performance-related characteristics. PhD thesis, University of Chester, https://chesterrep.openrepository.com/handle/ $10034 / 623170$

[766] Starling LT, Nellemann S, Parkes A, Lambert MI (2020) The Fatigue and Fitness Test for Teams (FFITT): A practical option for monitoring athletes in a team as individuals. European Journal of Sport Science 20(1):106-114, https://doi.org/ $10.1080 / 17461391.2019 .1612951$

[767] Steele J (2020) What is (perception of) effort? Objective and subjective effort during task performance. PsyArXiv https://pure.solent.ac.uk/ en/publications/what-is-perception-of-effortobjective-and-subjective-effort-duri

[768] Stein S (2021) Mark Zuckerberg on Facebook's VR future: New sensors on Quest Pro, fitness and a metaverse for work. CNET https://ww. cnet.com/features/mark-zuckerbergon-facebook-vr-future-new-sens ors - on-questpro-fitness-and-a-metaverse-for-work/

[769] Stergiou N, Decker LM (2011) Human movement variability, nonlinear dynamics, and pathology: Is there a connection? Human Movement Science 30(5):869-888, https://doi.org/10.1016/ j.humov.2011.06.002

[770] Sterzentsenko V, Doumanoglou A, Thermos S, Zioulis N, Zarpalas D, Daras P (2020) Deep Soft Procrustes for Markerless Volumetric Sensor Alignment. In: 2020 IEEE Conference on Virtual Reality and 3D User Interfaces (VR), pp 818-827

[771] Stillman CM, Cohen J, Lehman ME, Erickson KI (2016) Mediators of Physical Activity on Neurocognitive Function: A Review at Multiple Levels of Analysis. Frontiers in Human Neuroscience 10, https: //doi.org/10.3389/fnhum.2016.00626

[772] Stone JE, Postnova S, Sletten TL, Rajaratnam SMW, Phillips AJK (2020) Computational approaches for individual circadian phase prediction in field settings. Current Opinion in Systems Biology 22:39-51, https: //doi.org/10.1016/j.coisb.2020.07.011

[773] Strathern M (1997) 'Improving ratings': audit in the British University system. European Review $\quad 5(3): 305-321, \quad$ https://doi.org/ 
10.1002/(SICI) 1234-981X (199707) 5:3\%3C305: AID-EUR0184\%3E3.0.C0;2-4

[774] Strömbäck D, Huang S, Radu V (2020) MM-Fit: Multimodal Deep Learning for Automatic Exercise Logging across Sensing Devices. Proceedings of the ACM on Interactive, Mobile, Wearable and Ubiquitous Technologies 4(4):168:1-168:22, https: //doi.org/10.1145/3432701

[775] Su SY, Yu F, Zollhoefer M, Rhodin H (2021) A-NeRF : Surface-free Human 3D Pose Refinement via Neural Rendering. arXiv:210206199 [cs] http://arxiv.org/ abs/2102.06199

[776] Subbaswamy A, Saria S (2020) From development to deployment: dataset shift, causality, and shift-stable models in health AI. Biostatistics 21(2):345-352, https://doi.org/10.1093/biostatistics/kxz041

[777] Subin S (2021) Jeff Bezos is obsessed with a common Amazon warehouse injury. CNBC https://ww. cnbc.com/2021/04/25/jeff-bezosis-obsessed-with-a-common-amazon-warehouseinjury-.html

[778] Suchomel TJ, Nimphius S, Stone MH (2016) The Importance of Muscular Strength in Athletic Performance. Sports Medicine 46(10):1419-1449, https : //doi.org/10.1007/s40279-016-0486-0

[779] Sun C, Shrivastava A, Singh S, Gupta A (2017) Revisiting Unreasonable Effectiveness of Data in Deep Learning Era. arXiv:170702968 [cs] http:// arxiv.org/abs/1707.02968

[780] Sun Y, Zhang L, Schaeffer H (2019) NeuPDE: Neural Network Based Ordinary and Partial Differential Equations for Modeling Time-Dependent Data. arXiv:190803190 [cs, stat] http://arxiv.org/ abs/1908.03190

[781] Sung E, Han A, Hinrichs T, Vorgerd M, Manchado C, Platen P (2014) Effects of follicular versus luteal phase-based strength training in young women. SpringerPlus 3, https://dx.doi.org/10.1186/ 2193-1801-3-668

[782] Surís D, Liu R, Vondrick C (2021) Learning the Predictability of the Future. arXiv:210101600 [cs, eess] http://arxiv.org/abs/2101.01600

[783] Symul L, Holmes S (2021) Labeling selftracked menstrual health records with hidden semi-Markov models. medRxiv https: //doi.org/10.1101/2021.01.11.21249605

[784] Taborri J, Keogh J, Kos A, Santuz A, Umek A, Urbanczyk C, van der Kruk E, Rossi S (2020) Sport Biomechanics Applications Using Inertial, Force, and EMG Sensors: A Literature Overview. https:// doi.org/10.1155/2020/2041549

[785] Tack C (2019) Artificial intelligence and machine learning | applications in musculoskeletal physiotherapy. Musculoskeletal Science and Practice 39:164169, https://doi.org/10.1016/j.msksp.2018.11.012

[786] Taetz B, Teufl W, Weidmann A, Pietschmann J, Jöllenbeck T, Bleser G (2020) Depth camera based statistical shape fitting approach for the creation of an individualized lower body biomechanical model: validity and reliability. Computer Methods in Biomechanics and Biomedical Engineering 23(1):12-22, https://doi.org/10.1080/10255842.2019.1688310

[787] Takala TM, Hirao Y, Morikawa H, Kawai T (2020) Martial Arts Training in Virtual Reality with Fullbody Tracking and Physically Simulated Opponents. In: 2020 IEEE Conference on Virtual Reality and 3D User Interfaces Abstracts and Workshops (VRW), pp 858-858

[788] Takasu NN, Toichi M, Nakamura W (2011) Importance of regular lifestyle with daytime bright light exposure on circadian rhythm sleep-wake disorders in pervasive developmental disorders. Japanese Dental Science Review 47(2):141-149, https:// doi.org/10.1016/j.jdsr.2011.04.001

[789] Taksler GB, Dalton JE, Perzynski AT, Rothberg MB, Milinovich A, Krieger NI, Dawson NV, Roach MJ, Lewis MD, Einstadter D (2021) Opportunities, Pitfalls, and Alternatives in Adapting Electronic Health Records for Health Services Research. Medical Decision Making 41(2):133-142, https://doi.org/ 10.1177/0272989x20954403

[790] Talib I, Sundaraj K, Lam CK, Ali MA, Hussain J (2019) Mechanomyography: An Insight to Muscle Physiology. In: Intelligent Manufacturing and Mechatronics, Springer, Singapore, pp 129-137, https://doi.org/10.1007/978-981-13-9539-0_13

[791] Tang CI, Perez-Pozuelo I, Spathis D, Brage S, Wareham N, Mascolo C (2021) SelfHAR: Improving Human Activity Recognition through Self-training with Unlabeled Data. In: Proc. on Interactive, Mobile, Wearable and Ubiquitous Technologies (IMWUT/Ubicomp), vol 1, p 30

[792] Tanno R, Saeedi A, Sankaranarayanan S, Alexander DC, Silberman N (2019) Learning From Noisy Labels By Regularized Estimation Of Annotator Confusion. arXiv:190203680 [cs, stat] http://arxiv.org/ $\mathrm{abs} / 1902.03680$

[793] Tanrıkut E, Özcan \I, Sel E, Köytepe S, Savan EK (2020) Simultaneous Electrochemical Detection of Estradiol and Testosterone Using Nickel Ferrite Oxide Doped Mesoporous Carbon Nanocomposite Modified Sensor. Journal of The Electrochemical Society 167(8):087509, https://doi.org/10.1149/1945$7111 / \mathrm{ab} 927 f$

[794] Tanwani AK, Sermanet P, Yan A, Anand R, Phielipp M, Goldberg K (2020) Motion2Vec: Semi-Supervised Representation Learning from Surgical Videos. In: 2020 IEEE International Conference on Robotics and Automation (ICRA), pp 2174-2181, https:// doi.org/10.1109/ICRA40945.2020.9197324

[795] Tao J, Dong M, Li L, Wang C, Li J, Liu Y, Bao R, Pan C (2020) Real-time pressure mapping smart insole system based on a controllable vertical pore dielectric layer. Microsystems \& Nanoengineering 6(1):110, https://doi.org/10.1038/s41378-020-0171-1

[796] Tatariants M (2020) Challenges of Human Pose Estimation in AI-Powered Fitness Apps. InfoQ https://www.infoq.com/articles/human-poseestimation-ai-powered-fitness-apps /

[797] Taylor PE, Almeida GJM, Kanade T, Hodgins JK (2010) Classifying human motion quality for knee osteoarthritis using accelerometers. In: 2010 Annual International Conference of the IEEE Engineering in Medicine and Biology, pp 339-343

[798] Taylor WR, Schütz P, Bergmann G, List R, Postolka B, Hitz M, Dymke J, Damm P, Duda G, Gerber H, Schwachmeyer V, Hosseini Nasab SH, Trepczynski A, Kutzner I (2017) A comprehensive assessment of the musculoskeletal system: The CAMS-Knee data set. Journal of Biomechanics 65:32-39, https://doi.org/ 10.1016/j.jbiomech.2017.09.022

[799] Tedesco S, Crowe C, Ryan A, Sica M, Scheurer S, Clifford AM, Brown KN, O'Flynn B (2020) Motion Sensors-Based Machine Learning Approach for the Identification of Anterior Cruciate Ligament Gait Patterns in On-the-Field Activities in Rugby Players. Sensors 20(11):3029, https://doi.org/10.3390/ s20113029

[800] Teikari P, Najjar RP, Schmetterer L, Milea D (2019) Embedded deep learning in ophthalmology: making ophthalmic imaging smarter. Therapeutic Advances in Ophthalmology 11:2515841419827172, https:// doi.org/10.1177/2515841419827172

[801] Tenan MS, Vigotsky AD, Caldwell AR (2020) Comment on: "A Method to Stop Analyzing Random Error and Start Analyzing Differential Responders 
to Exercise". Sports Medicine 50(2):431-434, https: //doi.org/10.1007/s40279-019-01249-9

[802] Terse-Thakoor T, Punjiya M, Matharu Z, Lyu B, Ahmad M, Giles GE, Owyeung R, Alaimo F, Shojaei Baghini M, Brunyé TT, Sonkusale S (2020) Thread-based multiplexed sensor patch for real-time sweat monitoring. npj Flexible Electronics 4(1):1-10, https://doi.org/10.1038/s41528-020-00081-w

[803] Teufl W, Miezal M, Taetz B, Fröhlich M, Bleser G (2019) Validity of inertial sensor based 3D joint kinematics of static and dynamic sport and physiotherapy specific movements. PLOS ONE 14(2):e0213064, https://doi.org/10.1371/journal.pone.0213064

[804] Thambawita V, Hicks SA, Borgli H, Stensland HK, Jha D, Svensen MK, Pettersen SA, Johansen D, Johansen HD, Pettersen SD, Nordvang S, Pedersen S, Gjerdrum A, Grønli TM, Fredriksen PM, Eg R, Hansen K, Fagernes S, Claudi C, Biørn-Hansen A, Nguyen DTD, Kupka T, Hammer HL, Jain R, Riegler MA, Halvorsen P (2020) PMData: a sports logging dataset. In: Proceedings of the 11th ACM Multimedia Systems Conference, Association for Computing Machinery, New York, NY, USA, MMSys '20, pp 231236, https://doi.org/10.1145/3339825.3394926

[805] Thamm A, Freitag N, Figueiredo P, Doma K, Rottensteiner C, Bloch W, Schumann M (2019) Can Heart Rate Variability Determine Recovery Following Distinct Strength Loadings? A Randomized CrossOver Trial. International Journal of Environmental Research and Public Health 16(22):4353, https:// doi.org/10.3390/ijerph16224353

[806] Tharatipyakul A, Choo K, Perrault ST (2020) Pose Estimation for Facilitating Movement Learning from Online Videos. Proceedings of the International Conference on Advanced Visual Interfaces pp 1-5, http: //arxiv.org/abs/2004.0320s

[807] Thatipelli A, Trivedi N, Sarvadevabhatla RK (2021) NTU60-X: Towards Skeleton-based Recognition of Subtle Human Actions. arXiv:210111529 [cs] http: //arxiv.org/abs/2101.11529

[808] Thewlis D, Bishop C, Daniell N, Paul G (2013) NextGeneration Low-Cost Motion Capture Systems Can Provide Comparable Spatial Accuracy to High-End Systems. Journal of Applied Biomechanics 29(1):112117, https://doi.org/10.1123/jab.29.1.112

[809] Thiel A, Sudeck G, Gropper H, Maturana FM, Schubert T, Srismith D, Widmann M, Behrens S, Martus P, Munz B, Giel K, Zipfel S, Nieß AM (2020) The iReAct study - A biopsychosocial analysis of the individual response to physical activity. Contemporary Clinical Trials Communications 17:100508, https://doi.org/10.1016/j.conctc.2019.100508

[810] Thompson SW, Rogerson D, Ruddock A, Barnes A (2020) The Effectiveness of Two Methods of Prescribing Load on Maximal Strength Development: A Systematic Review. Sports Medicine 50(5):919-938, https://doi.org/10.1007/s40279-019-01241-3

[811] Thorpe H, Brice J, Clark M (2020) Digital Intimacies, Assemblages, and Fit Femininities. In: Thorpe H, Brice J, Clark M (eds) Feminist New Materialisms, Sport and Fitness: A Lively Entanglement, New Femininities in Digital, Physical and Sporting Cultures, Springer International Publishing, Cham, pp 91-118, https://doi.org/10.1007/978-3-030-56581-7_4

[812] Tiase VL, Hull W, McFarland MM, Sward KA, Del Fiol G, Staes C, Weir C, Cummins MR (2020) Patient-generated health data and electronic health record integration: a scoping review. JAMIA Open (ooaa052), https://doi.org/10.1093/ jamiaopen/ooaa052

[813] Ticinesi A, Meschi T, Narici MV, Lauretani F, Maggio M (2017) Muscle Ultrasound and Sarcopenia in Older Individuals: A Clinical Perspect- ive. Journal of the American Medical Directors Association 18(4):290-300, https://doi.org/10.1016/ j.jamda.2016.11.013

[814] Tinsley GM, Moore ML, Dellinger JR, Adamson BT, Benavides ML (2020) Digital anthropometry via three-dimensional optical scanning: evaluation of four commercially available systems. European Journal of Clinical Nutrition 74(7):1054-1064, https : //doi.org/10.1038/s41430-019-0526-6

[815] Toelle TR, Utpadel-Fischler DA, Haas KK, Priebe JA (2019) App-based multidisciplinary back pain treatment versus combined physiotherapy plus online education: a randomized controlled trial. npj Digital Medicine 2(1):1-9, https://doi.org/10.1038/ s41746-019-0109-x

[816] Tome D, Toso M, Agapito L, Russell C (2018) Rethinking Pose in 3D: Multi-stage Refinement and Recovery for Markerless Motion Capture. In: 2018 International Conference on 3D Vision (3DV), pp 474-483, https://doi.org/10.1109/3DV.2018.00061

[817] Topley M, Richards JG (2020) A comparison of currently available optoelectronic motion capture systems. Journal of Biomechanics 106:109820, https: //doi.org/10.1016/j.jbiomech.2020.109820

[818] Trajkova M (2020) Designing AI-Based Feedback for Ballet Learning. In: Extended Abstracts of the 2020 CHI Conference on Human Factors in Computing Systems, Association for Computing Machinery, New York, NY, USA, CHI EA '20, pp 1-9, https: //doi.org/10.1145/3334480.3375036

[819] Travis SK, Goodin JR, Beckham GK, Bazyler CD (2018) Identifying a Test to Monitor Weightlifting Performance in Competitive Male and Female Weightlifters. Sports 6(2):46, https://doi.org/ $10.3390 /$ sports 6020046

[820] Travis SK, Ishida A, Taber CB, Fry AC, Stone MH (2020) Emphasizing Task-Specific Hypertrophy to Enhance Sequential Strength and Power Performance. Journal of Functional Morphology and Kinesiology 5(4):76, https://doi.org/10.3390/jfmk5040076

[821] Travis SK, Mujika I, Gentles JA, Stone MH, Bazyler CD (2020) Tapering and Peaking Maximal Strength for Powerlifting Performance: A Review. Sports 8(9):125, https://doi.org/10.3390/sports 8090125

[822] Trinidad-Fernández M, Beckwée D, Cuesta-Vargas A, González-Sánchez M, Moreno FÁ, González-Jiménez J, Joos E, Vaes P (2021) Differences in movement limitations in different low back pain severity in functional tests using an RGB-D camera. Journal of Biomechanics 116:110212, https://doi.org/10.1016/ j.jbiomech.2020.110212

[823] Truong N, Sun K, Wang S, Guitton F, Guo Y (2020) Privacy Preservation in Federated Learning: Insights from the GDPR Perspective. arXiv:201105411 [cs] http://arxiv.org/abs/2011.05411

[824] Tuchscherer M (2008) The Reactive Training Manual: Developing your own custom training program for powerlifting. No. 15 in Reactive Training Systems, Michael Tuchscherer

[825] Tuka V, Linhart A (2020) Personalised exercise prescription: Finding the best for our patients. European Journal of Preventive Cardiology 27(13):1366-1368, https://doi.org/10.1177/2047487319884376

[826] Tumnark P (2018) Ontology-based personalized performance evaluation and dietary recommendation for weightlifting. Doctoral thesis, University of Porto, https://hdl.handle.net/10216/116559

[827] Turmo Vidal L, Márquez Segura E, Parrilla Bel L, Waern A (2020) Training Technology Probes Across Fitness Practices: Yoga, Circus and Weightlifting. In: Extended Abstracts of the 2020 CHI Conference on Human Factors in Computing Systems, As- 
sociation for Computing Machinery, New York, NY, USA, CHI EA '20, pp 1-8, https://doi.org/10.1145/ 3334480.3382862

[828] Turmo Vidal L, Zhu H, Riego-Delgado A (2020) BodyLights: Open-Ended Augmented Feedback to Support Training Towards a Correct Exercise Execution. In: Proceedings of the $2020 \mathrm{CHI}$ Conference on Human Factors in Computing Systems, Association for Computing Machinery, New York, NY, USA, pp 1-14, https://doi.org/10.1145/3313831.3376268

[829] Turner AN, Comfort P, McMahon J, Bishop C, Chavda S, Read P, Mundy P, Lake J (2020) Developing Powerful Athletes, Part 1: Mechanical Underpinnings. Strength \& Conditioning Journal 42(3):30-39, http://doi.org/10.1519/SSC.0000000000000543

[830] Umehara J, Nakamura M, Saeki J, Tanaka H, Yanase K, Fujita K, Yamagata M, Ichihashi N (2021) Acute and Prolonged Effects of Stretching on Shear Modulus of the Pectoralis Minor Muscle. Journal of Sports Science and Medicine 20(1):17-25, https:// Www.jssm.org/abstresearchajssm-20-17.xml.xml

[831] Upadhyay D, Pandey V, Nag N, Jain R (2020) N=1 Modelling of Lifestyle Impact on SleepPerformance. arXiv:200610884 [cs, q-bio] http://arxiv.org/abs/ 2006.10884

[832] Urteaga I, McKillop M, Elhadad N (2020) Learning endometriosis phenotypes from patient-generated data. npj Digital Medicine 3(1):1-14, https:// doi.org/10.1038/s41746-020-0292-9

[833] Vaith A, Taetz B, Bleser G (2020) Uncertainty based active learning with deep neural networks for inertial gait analysis. In: 2020 IEEE 23rd International Conference on Information Fusion (FUSION), pp 1-8, https://doi.org/10.23919/ FUSION45008.2020.9190449

[834] Vakanski A, Jun Hp, Paul D, Baker R (2018) A Data Set of Human Body Movements for Physical Rehabilitation Exercises. Data 3(1):2, https://doi.org/ 10.3390/data3010002

[835] Valcik J, Sedmidubsky J, Zezula P (2016) Assessing similarity models for human-motion retrieval applications. Computer Animation and Virtual Worlds 27(5):484-500, https://doi.org/10.1002/cav.1674

[836] Vasudevan RK, Ziatdinov M, Vlcek L, Kalinin SV (2021) Off-the-shelf deep learning is not enough, and requires parsimony, Bayesianity, and causality. npj Computational Materials 7(1):1-6, https://doi.org/ $10.1038 / \mathrm{s} 41524-020-00487-0$

[837] Veniat T, Denoyer L, Ranzato M (2021) Efficient Continual Learning with Modular Networks and Task-Driven Priors. arXiv:201212631 [cs] http:// arxiv.org/abs/2012.12631

[838] Vesterinen V, Nummela A, Heikura I, Laine $T$, Hynynen E, Botella J, Häkkinen K (2016) Individual Endurance Training Prescription with Heart Rate Variability. Medicine \& Science in Sports \& Exercise 48(7):1347-1354, http://doi.org/10.1249/ MSS. 0000000000000910

[839] Vidal Pérez D, Martínez-Sanz JM, Ferriz-Valero A Gómez-Vicente V, Ausó E (2021) Relationship of Limb Lengths and Body Composition to Lifting in Weightlifting. International Journal of Environmental Research and Public Health 18(2):756, https: //doi.org/10.3390/ijerph18020756

[840] Vigotsky AD (2015) Can motor unit recruitment be inferred from EMG amplitude? https://bretcontreras.com/can-motor-unitrecruitment-be-inferred-from-emg-amplitude/

[841] Vigotsky AD, Halperin I, Lehman GJ, Trajano GS, Vieira TM (2018) Interpreting Signal Amplitudes in Surface Electromyography Studies in Sport and Rehabilitation Sciences. Frontiers in Physiology 8, https://doi.org/10.3389/fphys.2017.00985
[842] Vigotsky AD, Bryanton MA, Nuckols G, Beardsley C, Contreras B, Evans J, Schoenfeld BJ (2019) Biomechanical, Anthropometric, and Psychological Determinants of Barbell Back Squat Strength. Journal of Strength and Conditioning Research 33 Suppl 1:S26S35, http://doi.org/10.1519/JSC.0000000000002535

[843] Vingren JL, Kraemer WJ, Ratamess NA, Anderson JM, Volek JS, Maresh CM (2010) Testosterone Physiology in Resistance Exercise and Training. Sports Medicine 40(12):1037-1053, https://doi.org/ 10.2165/11536910-000000000-00000

[844] Vlahoyiannis A, Aphamis G, Bogdanis GC, Sakkas GK, Andreou E, Giannaki CD (2020) Deconstructing athletes' sleep: A systematic review of the influence of age, sex, athletic expertise, sport type, and season on sleep characteristics. Journal of Sport and Health Science https://doi.org/10.1016/j.jshs.2020.03.006

[845] Vyas P (2019) POSE ESTIMATION AND ACTION RECOGNITION IN SPORTS AND FITNESS. Master's Projects https://doi.org/10.31979/etd.w8ug$4 \mathrm{v} 5 \mathrm{c}$

[846] Wallace BJ, Bergstrom HC, Butterfield TA (2018) Muscular bases and mechanisms of variable resistance training efficacy. International Journal of Sports Science \& Coaching 13(6):1177-1188, https://doi.org/ 10.1177/1747954118810240

[847] Walsh NP, Halson SL, Sargent C, Roach GD, Nédélec M, Gupta L, Leeder J, Fullagar HH, Coutts AJ, Edwards BJ, Pullinger SA, Robertson CM, Burniston JG, Lastella M, Meur YL, Hausswirth C, Bender AM, Grandner MA, Samuels CH (2020) Sleep and the athlete: narrative review and 2021 expert consensus recommendations. British Journal of Sports Medicine http://dx.doi.org/10.1136/bjsports-2020-102025

[848] Walton CC, Liknaitzky P (2020) Advancing elite athlete mental health treatment with psychedelic-assisted psychotherapy. Journal of Applied Sport Psychology 0(0):1-19, https://doi.org/10.1080/10413200.2020.1848941

[849] Wan C, McHill AW, Klerman E, Sano A (2020) Sensor-Based Estimation of Dim Light Melatonin Onset (DLMO) Using Features of Two Time Scales. arXiv:190807483 [cs, eess, stat] http://arxiv.org/ abs/1908.07483

[850] Wang B, Yang $\mathrm{K}$, Cheng $\mathrm{H}$, Ye $\mathrm{T}$, Wang $\mathrm{C}$ (2021) A hydrophobic conductive strip with outstanding one-dimensional stretchability for wearable heater and strain sensor. Chemical Engineering Journal 404:126393, https://doi.org/10.1016/ j.cej.2020.126393

[851] Wang C, Vargas JT, Stokes T, Steele R, Shrier I (2020) Analyzing Activity and Injury: Lessons Learned from the Acute:Chronic Workload Ratio. Sports Medicine 50(7):1243-1254, https://doi.org/ $10.1007 / \mathrm{s} 40279-020-01280-1$

[852] Wang CKJ, Tan L, Dairianathan EI (2018) Achievement Goals, Implicit Theories, and Intrinsic Motivation: A Test of Domain Specificity Across Music, Visual Art, and Sports. Journal of Research in Music Education 66(3):320-337, https://doi.org/10.1177/ 0022429418784563

[853] Wang H, Liu Q, Yue X, Lasenby J, Kusner MJ (2020) Pre-Training by Completing Point Clouds. arXiv:201001089 [cs] http://arxiv.org/abs / 2010.01089

[854] Wang K, Yap LW, Gong S, Wang R, Wang SJ, Cheng W (2021) Nanowire-Based Soft Wearable Human-Machine Interfaces for Future Virtual and Augmented Reality Applications. Advanced Functional Materials n/a(n/a):2008347, https://doi.org/ 10.1002/adfm.202008347

[855] Wang L, Zhang W, He X, Zha H (2018) Supervised Reinforcement Learning with Recurrent Neural 
Network for Dynamic Treatment Recommendation. In: Proceedings of the 24th ACM SIGKDD International Conference on Knowledge Discovery \& Data Mining, Association for Computing Machinery, New York, NY, USA, KDD '18, pp 2447-2456, https: //doi.org/10.1145/3219819.3219961

[856] Wang L, Sun B, Robinson J, Jing T, Fu Y (2020) EV-Action: Electromyography-Vision MultiModal Action Dataset. arXiv:190412602 [cs] http: //arxiv.org/abs/1904.12602

[857] Wang Q, Kurillo G, Ofli F, Bajcsy R (2015) Unsupervised Temporal Segmentation of Repetitive Human Actions Based on Kinematic Modeling and Frequency Analysis. In: 2015 International Conference on 3D Vision, pp 562-570

[858] Wang W, Tran D, Feiszli M (2020) What Makes Training Multi-Modal Classification Networks Hard? In: CVPR 2020, pp 12695-12705, https:// arxiv.org/abs/1905.12681

[859] Wang Y (2020) An Object-oriented Software Framework for Immersive Virtual Reality Exergames. Doctoral thesis, University of Sydney, https:// ses.library.usyd.edu.au/handle/2123/21986

[860] Weakley J, Chalkley D, Johnston R, García-Ramos A, Townshend A, Dorrell H, Pearson M, Morrison M, Cole M (2020) Criterion Validity, and Interunit and Between-Day Reliability of the FLEX for Measuring Barbell Velocity During Commonly Used Resistance Training Exercises. The Journal of Strength \& Conditioning Research 34(6):1519-1524, http://doi.org/ $10.1519 /$ JSC .0000000000003592

[861] Weakley J, Mann B, Banyard H, McLaren S, Scott T, Garcia-Ramos A (2020) Velocity-Based Training: From Theory to Application. Strength \& Conditioning Journal Publish Ahead of Print, http://doi.org/ 10.1519/SSC.0000000000000560

[862] Weakley J, McLaren S, Ramirez-Lopez C, GarcíaRamos A, Dalton-Barron N, Banyard H, Mann B, Weaving D, Jones B (2020) Application of velocity loss thresholds during free-weight resistance training: Responses and reproducibility of perceptual, metabolic, and neuromuscular outcomes. Journal of Sports Sciences 38(5):477-485, https://doi.org/ 10.1080/02640414.2019.1706831

[863] Weidmann A, Taetz B, Andres M, Laufer F, Bleser G (2020) Force Shadows: An Online Method to Estimate and Distribute Vertical Ground Reaction Forces from Kinematic Data. Sensors 20(19):5709, https: //doi.org/10.3390/s20195709

[864] Weizman Y, Tan AM, Fuss FK (2019) Benchmarking study of the forces and centre of pressure derived from a novel smart-insole against an existing pressure measuring insole and force plate. Measurement 142:48-59, https://doi.org/10.1016/ j.measurement.2019.03.023

[865] Welch Bacon CE, Pike Lacy AM, Lam KC (2021) Knowledge Translation in Athletic Training: Considerations for Bridging the Knowledge-to-Practice Gap. Journal of Athletic Training https://doi.org/ $10.4085 / 0470-20$

[866] Weng C, Shah NH, Hripcsak G (2020) Deep phenotyping: Embracing complexity and temporality-Towards scalability, portability, and interoperability. Journal of Biomedical Informatics 105:103433, https://dx.doi.org/10.1016/j.jbi.2020.103433

[867] Westcott WL (2012) Resistance Training is Medicine: Effects of Strength Training on Health. Current Sports Medicine Reports 11(4):209-216, http: //doi.org/10.1249/JSR.0b013e31825dabb8

[868] Weygers I, Kok M, Konings M, Hallez H, De Vroey H, Claeys K (2020) Inertial Sensor-Based Lower Limb Joint Kinematics: A Methodological Systematic Review. Sensors 20(3):673, https://doi.org/10.3390/ s20030673

[869] Whitaker J (2020) Completing the Machine Learning Loop. https://jimmymwhitaker medium.com/ completing-the-machine-learning-loope03c784eaab4

[870] Wiedemann LG, Planinc R, Nemec I, Kampel M (2015) Performance evaluation of joint angles obtained by the Kinect v2. In: IET International Conference on Technologies for Active and Assisted Living (TechAAL), pp $6 .-6$., https://doi.org/10.1049/ ic. 2015.0142

[871] Wiehr F, Vujic M, Krüger A, Daiber F (2020) The Jungle Warm-Up Run: Augmenting Athletes with Coach-Guided Dynamic Game Elements. In: Proceedings of the Augmented Humans International Conference, Association for Computing Machinery, New York, NY, USA, AHs '20, pp 1-12, https: //doi.org/10.1145/3384657.3384779

[872] Wikström-Frisén L, Boraxbekk CJ, HenrikssonLarsén K (2017) Effects on power, strength and lean body mass of menstrual/oral contraceptive cycle based resistance training. The Journal of Sports Medicine and Physical Fitness 57(1-2):43-52, https: //doi.org/10.23736/s0022-4707.16.05848-5

[873] Wilkinson J, Arnold KF, Murray EJ, van Smeden M, Carr K, Sippy R, de Kamps M, Beam A, Konigorski S, Lippert C, Gilthorpe MS, Tennant PWG (2020) Time to reality check the promises of machine learning-powered precision medicine. The Lancet Digital Health 2(12):e677-e680, https://doi.org/ $10.1016 /$ S2589-7500 (20)30200-4

[874] Willby N (2019) Strength and Conditioning for Pole. The Pole PT Limited, https://www.thepolept.com/ the-book/

[875] Williams TD, Tolusso DV, Fedewa MV, Esco MR (2017) Comparison of Periodized and Non-Periodized Resistance Training on Maximal Strength: A Meta-Analysis. Sports Medicine (Auckland, NZ) 47(10):2083-2100, https://doi.org/10.1007/s40279-017-0734-y

[876] Williams TD, Esco MR, Fedewa MV, Bishop PA (2020) Bench Press Load-Velocity Profiles and Strength After Overload and Taper Microcyles in Male Powerlifters. The Journal of Strength \& Conditioning Research 34(12):3338-3345, http://doi.org/ 10.1519/JSC. 0000000000003835

[877] Williams TD, Esco MR, Fedewa MV, Bishop PA (2021) Inter- and Intra-Day Comparisons of Smartphone-Derived Heart Rate Variability across Resistance Training Overload and Taper Microcycles. International Journal of Environmental Research and Public Health 18(1):177, https://doi.org/10.3390/ ijerph18010177

[878] Willick SE, Cushman DM, Blauwet CA, Emery C, Webborn N, Derman W, Schwellnus M, Stomphorst J, Vliet PVd (2016) The epidemiology of injuries in powerlifting at the London 2012 Paralympic Games: An analysis of 1411 athlete-days. Scandinavian Journal of Medicine \& Science in Sports 26(10):1233-1238, https://doi.org/10.1111/ sms. 12554

[879] Winchester JB, Erickson TM, Blaak JB, McBride JM (2005) Changes in bar-path kinematics and kinetics after power-clean training. Journal of Strength and Conditioning Research 19(1):177-183, https:// doi.org/10.1519/14023.1

[880] Winchester JB, Porter JM, McBride JM (2009) Changes in Bar Path Kinematics and Kinetics Through Use of Summary Feedback in Power Snatch Training. The Journal of Strength \& Conditioning Research 23(2):444-454, http://sci-hub.se/ 10.1519/JSC.0b013e318198fc73

[881] Windt J, Ardern CL, Gabbett TJ, Khan KM, 
Cook CE, Sporer BC, Zumbo BD (2018) Getting the most out of intensive longitudinal data: a methodological review of workload-injury studies. BMJ Open 8(10):e022626, https ://bmjopen.bmj.com/ content/8/10/e022626

[882] Windt J, MacDonald K, Taylor D, Zumbo BD, Sporer BC, Martin DT (2020) "To Tech or Not to Tech?" A Critical Decision-Making Framework for Implementing Technology in Sport. Journal of Athletic Training 55(9):902-910, https://doi.org/10.4085/1062$6050-0540.19$

[883] Wing C (2018) Monitoring Athlete Load: Data Collection Methods and Practical Recommendations. Strength \& Conditioning Journal 40(4):26-39, http: //doi.org/10.1519/SSC.0000000000000384

[884] Winkelmann ZK, Eberman LE, Games KE (2020) Telemedicine Experiences of Athletic Trainers and Orthopaedic Physicians for Patients With Musculoskeletal Conditions. Journal of Athletic Training 55(8):768-779, https://doi.org/10.4085/1062$6050-388-19$

[885] Woelfel JR, Dudley-Javoroski S, Shields RK (2018) Precision Physical Therapy: Exercise, the Epigenome, and the Heritability of Environmentally Modified Traits. Physical Therapy 98(11):946-952, https: //doi.org/10.1093/ptj/pzy092

[886] Wood RI, Stanton SJ (2012) Testosterone and sport: Current perspectives. Hormones and Behavior 61(1):147-155, https: //doi.org/10.1016/j.yhbeh.2011.09.010

[887] Woods CT, Rothwell M, Rudd J, Robertson S, Davids K (2021) Representative co-design: Utilising a source of experiential knowledge for athlete development and performance preparation. Psychology of Sport and Exercise 52:101804, https://doi.org/10.1016/ j.psychsport.2020.101804

[888] Worsey MT, Espinosa HG, Shepherd JB, Thiel DV (2019) Inertial Sensors for Performance Analysis in Combat Sports: A Systematic Review. Sports $7(1): 28$, https://doi.org/10.3390/sports7010028

[889] Wu M, Goodman N (2018) Multimodal Generative Models for Scalable Weakly-Supervised Learning. arXiv:180205335 [cs, stat] http://arxiv.org/ abs / 1802.05335

[890] Wu W, Saul K, Huang HH (2020) Using Reinforcement Learning to Estimate Human Joint Moments via EMG Signals or Joint Kinematics: An Alternative Solution to Musculoskeletal-Based Biomechanics. Journal of Biomechanical Engineering https:// doi.org/10.1115/1.4049333

[891] Xiao ZG, Menon C (2019) A Review of Force Myography Research and Development. Sensors 19(20):4557, https://doi.org/10.3390/s19204557

[892] Xie T, France-Lanord A, Wang Y, Shao-Horn Y, Grossman JC (2019) Graph dynamical networks for unsupervised learning of atomic scale dynamics in materials. Nature Communications 10(1):2667, https://doi.org/10.1038/s41467-019-10663-6

[893] Xiong Y, Tao X (2018) Compression Garments for Medical Therapy and Sports. Polymers 10(6):663, https://doi.org/10.3390/polym10060663

[894] Xu PA, Mishra AK, Bai H, Aubin CA, Zullo L, Shepherd RF (2019) Optical lace for synthetic afferent neural networks. Science Robotics 4(34), http: //doi.org/10.1126/scirobotics aaw6304

[895] Xu X, Peng H, Sun L, Niu Y, Ma H, Liu L, He L (2021) Federated Depression Detection from MultiSource Mobile Health Data. arXiv:210209342 [cs] http://arxiv.org/abs/2102.09342

[896] Xu Z, Chen L, Lu L, Du R, Ma W, Cai Y, An $\mathrm{X}, \mathrm{Wu} \mathrm{H}$, Luo Q, Xu Q, Zhang Q, Jia X (2021) A Highly-Adhesive and Self-Healing Elastomer for Bio-Interfacial Electrode. Advanced Functional
Materials 31(1):2006432, https://doi.org/10.1002/ adfm.202006432

[897] Yan S, Xiong Y, Lin D (2018) Spatial Temporal Graph Convolutional Networks for Skeleton-Based Action Recognition. arXiv:180107455 [cs] http:// arxiv.org/abs/1801.07455

[898] Yang C, Wang X, Mao S (2020) RFID-Pose: VisionAided Three-Dimensional Human Pose Estimation With Radio-Frequency Identification. IEEE Transactions on Reliability pp 1-14, https://doi.org/ 10.1109/TR.2020.3030952

[899] Yao X, Sun L (2020) Continual Local Training for Better Initialization of Federated Models. 2020 IEEE International Conference on Image Processing (ICIP) pp 1736-1740, http://arxiv.org/abs/2005.12657

[900] Yasser A, Tariq D, Samy R, Hassan MA, Atia A (2019) Smart Coaching: Enhancing Weightlifting and Preventing Injuries. International Journal of Advanced Computer Science and Applications 10

[901] Yıldız Ç, Heinonen M, Lähdesmäki H (2019) ODE\$ $\$$ \$VAE: Deep generative second order ODEs with Bayesian neural networks. arXiv:190510994 [cs, stat] http: //arxiv.org/abs/1905.10994

[902] Yim J, Chopra R, Spitz T, Winkens J, Obika A, Kelly C, Askham H, Lukic M, Huemer J, Fasler K, Moraes G, Meyer C, Wilson M, Dixon J, Hughes C, Rees G, Khaw PT, Karthikesalingam A, King D, Hassabis D, Suleyman M, Back T, Ledsam JR, Keane PA, De Fauw J (2020) Predicting conversion to wet age-related macular degeneration using deep learning. Nature Medicine 26(6):892-899, https:// doi.org/10.1038/s41591-020-0867-7

[903] Yoon J, Jeong W, Lee G, Yang E, Hwang SJ (2020) Federated Continual Learning with Weighted Interclient Transfer. arXiv:200303196 [cs, stat] http:// arxiv.org/abs/2003.03196

[904] Yosinski J, Clune J, Bengio Y, Lipson H (2014) How transferable are features in deep neural networks? arXiv:14111792 [cs] http://arxiv.org/abs/ 1411.1792

[905] Young W, Talpey S, Bartlett R, Lewis M, Mundy S, Smyth A, Welsh T (2019) Development of Muscle Mass: How Much Is Optimum for Performance? Strength \& Conditioning Journal 41(3):47-50, http: //doi.org/10.1519/SSC.0000000000000443

[906] Young WB (2006) Transfer of strength and power training to sports performance. International Journal of Sports Physiology and Performance 1(2):74-83, https://doi.org/10.1123/ijspp.1.2.74

[907] Yuan M, Gao L, Fu H, Xia S (2019) Temporal Upsampling of Depth Maps Using a Hybrid Camera. IEEE Transactions on Visualization and Computer Graphics 25(3):1591-1602, https://doi.org/ 10.1109/TVCG.2018.2812879

[908] Yun S, Oh SJ, Heo B, Han D, Choe J, Chun S (2021) Re-labeling ImageNet: from Single to Multi-Labels, from Global to Localized Labels. arXiv:210105022 [cs] http: //arxiv.org/abs/2101.05022

[909] Zabat M, Ababou A, Ababou N, Dumas R (2019) IMU-based sensor-to-segment multiple calibration for upper limb joint angle measurement-a proof of concept. Medical \& Biological Engineering \& Computing 57(11):2449-2460, https://doi.org/10.1007/ s11517-019-02033-7

[910] Zamarayeva AM, Yamamoto NAD, Toor A, Payne ME, Woods C, Pister VI, Khan Y, Evans JW, Arias AC (2020) Optimization of printed sensors to monitor sodium, ammonium, and lactate in sweat. APL Materials 8(10):100905, https://doi.org/10.1063/ 5.0014836

[911] Zaras N, Stasinaki AN, Spiliopoulou P, Arnaoutis G, Hadjicharalambous M, Terzis G (2020) Rate of Force Development, Muscle Architecture, and Per- 
formance in Elite Weightlifters. International Journal of Sports Physiology and Performance -1(aop):1-8, https://doi.org/10.1123/ijspp.2019-0974

[912] Zatsiorsky VM, Kraemer WJ, Fry AC (2020) Science and Practice of Strength Training. Human Kinetics

[913] Zea M, Bellagambi FG, Ben Halima H, Zine N, Jaffrezic-Renault N, Villa R, Gabriel G, Errachid A (2020) Electrochemical sensors for cortisol detections: Almost there. TrAC Trends in Analytical Chemistry 132:116058, https://doi.org/10.1016/ j.trac. 2020.116058

[914] Zhang H, Liang J, Chen N (2020) Do not neglect the role of circadian rhythm in muscle atrophy. Ageing Research Reviews 63:101155, https://doi.org/ $10.1016 / j$.arr. 2020.101155

[915] Zhang J, Li W, Ogunbona PO, Wang P, Tang C (2016) RGB-D-based action recognition datasets: A survey. Pattern Recognition 60:86-105, https:// doi.org/10.1016/j.patcog.2016.05.019

[916] Zhang P, Lan C, Zeng W, Xing J, Xue J, Zheng N (2020) Semantics-Guided Neural Networks for Efficient Skeleton-Based Human Action Recognition. arXiv:190401189 [cs] http://arxiv.org/abs/ 1904.01189

[917] Zhang W, Zhu M, Derpanis KG (2013) From Actemes to Action: A Strongly-Supervised Representation for Detailed Action Understanding. In: 2013 IEEE International Conference on Computer Vision, pp 22482255

[918] Zhang X, Sun K, Guo Y (2021) OmiEmbed: reconstruct comprehensive phenotypic information from multi-omics data using multi-task deep learning. arXiv:210202669 [q-bioGN] https://arxiv.org/abs / 2102.02669v 1

[919] Zhang Y, Haghighi PD, Burstein F, Yap LW, Cheng W, Yao L, Cicuttini F (2020) Electronic Skin Wearable Sensors for Detecting Lumbar-Pelvic Movements. Sensors 20(5):1510, https://doi.org/ $10.3390 / \mathrm{s} 20051510$

[920] Zhang Z, Wang C, Qin W, Zeng W (2020) Fusing Wearable IMUs with Multi-View Images for Human Pose Estimation: A Geometric Approach. arXiv:200311163 [cs] http://arxiv.org/abs/ 2003.11163

[921] Zhang Z, Girdhar R, Joulin A, Misra I (2021) SelfSupervised Pretraining of 3D Features on any PointCloud. arXiv:210102691 [cs] http://arxiv.org/abs/ 2101.02691

[922] Zhao Z, Arya A, Orji R, Chan G (2020) Effects of a Personalized Fitness Recommender System Using Gamification and Continuous Player Modeling: System Design and Long-Term Validation Study. JMIR Serious Games 8(4):e19968, https:// games.jmir.org/2020/4/e19968

[923] Zheng C, Wu W, Yang T, Zhu S, Chen C, Liu
R, Shen J, Kehtarnavaz N, Shah M (2020) Deep Learning-Based Human Pose Estimation: A Survey. arXiv:201213392 [cs] http://arxiv.org/abs/ 2012.13392

[924] Zheng Y, Shin H, Kamper DG, Hu X (2021) Automatic Detection of Contracting Muscle Regions via the Deformation Field of Transverse Ultrasound Images: A Feasibility Study. Annals of Biomedical Engineering 49(1):354-366, https://doi.org/10.1007/ s 10439-020-02557-2

[925] Zhou M (2020) Designing ML Orchestration Systems for Startups. https://towardsdatascience.com/ designing-ml-orchestration-systems-forstartups-202e527d7897

[926] Zhou M, Fukuoka Y, Goldberg K, Vittinghoff E, Aswani A (2019) Applying machine learning to predict future adherence to physical activity programs. BMC Medical Informatics and Decision Making 19(1):169, https://doi.org/10.1186/s12911-019-0890-0

[927] Zhu K (2020) Augmented Reality for Exercises for Elderly People. Bachelor of Engineering, Metropolia University of Applied Sciences, Helsinki, Finland, http://urn.fi/URN:NBN : fi: amk-2020120626383

[928] Zhu L, Cao Q, Cai Y (2020) Development of augmented reality serious games with a vibrotactile feedback jacket. Virtual Reality \& Intelligent Hardware 2(5):454-470, https://doi.org/10.1016/ j.vrih.2020.05.005

[929] Zhu M, Memar AH, Gupta A, Samad M, Agarwal P, Visell Y, Keller SJ, Colonnese N (2020) PneuSleeve: In-fabric Multimodal Actuation and Sensing in a Soft, Compact, and Expressive Haptic Sleeve. In: Proceedings of the $2020 \mathrm{CHI}$ Conference on Human Factors in Computing Systems, Association for Computing Machinery, New York, NY, USA, CHI '20, pp 1-12, https://doi.org/10.1145/3313831.3376333

[930] Zhu R (2020) Application of Business Analysis in Sports Business. In: 2020 2nd International Conference on Economic Management and Cultural Industry (ICEMCI 2020), Atlantis Press, pp 129-132, https://dx.doi.org/10.2991/aebmr.k.201128.026

[931] Ziegelstein RC (2015) Personomics. JAMA Internal Medicine 175(6):888-889, https://doi.org/10.1001/ jama internmed.2015.0861

[932] Zoph B, Ghiasi G, Lin TY, Cui Y, Liu H, Cubuk ED, Le Q (2020) Rethinking Pre-training and Selftraining. Advances in Neural Information Processing Systems 33, https://arxiv.org/abs/2006.06882

[933] Zourdos MC, Klemp A, Dolan C, Quiles JM, Schau KA, Jo E, Helms E, Esgro B, Duncan S, Garcia Merino S, Blanco R (2016) Novel Resistance Training-Specific Rating of Perceived Exertion Scale Measuring Repetitions in Reserve. The Journal of Strength \& Conditioning Research 30(1):267-275, http://doi.org/10.1519/JSC.0000000000001049 